\title{
48691
}

Paper No. T.1.3

N $93-1<689$

\section{SHOCK/VORTEX INTERACTION AND VORTEX-BREAKDOWN MODES}

O. A. Kandil and H. A. Kandil

Old Dominion University, Norfolk, VA, USA

C. H. Liu

NASA Langley Research Center, Hampton, VA, USA

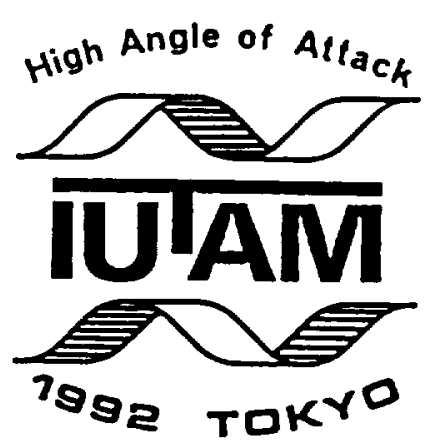

IUTAM SYMPOSIUM OF FLUID DYNAMICS OF HIGH ANGLE OF ATTACK Tokyo, Japan - September 13-17, 1992 



\title{
SHOCK/VORTEX INTERACTION AND VORTEX-BREAKDOWN MODES
}

\author{
O. A. Kandil and H. A. Kandil \\ Dept. of Mechanical Engineering and Mechanics \\ Old Dominion University, Norfolk, VA \\ C. H. Liu \\ Theoretical Flow Physics Br. \\ NASA Langley, Hampton, VA
}

\section{Summary}

Computational simulation and study of shock/vortex interaction and vortex-breakdown modes are considered for bound (internal) and unbound (external) flow domains. The problem is formulated using the unsteady, compressible, full Navier-Stokes (NS) equations which are solved using an implicit, flux-difference splitting, finite-volume scheme. For the bound flow domain, a supersonic swirling flow is considered in a configured circular duct and the problem is solved for quasi-axisymmetric and three-dimensional flows. For the unbound domain, a supersonic swirling flow issued from a nozzle into a uniform supersonic flow of lower Mach number is considered for quasi-axisymmetric and three-dimensional flows. The results show several modes of breakdown; e.g., no-breakdown, transient single-bubble breakdown, transient multi-bubble breakdown, periodic multi-bubble multi-frequency breakdown and helical breakdown.

\section{Introduction}

Longitudinal vortex/transverse shock-wave interactions are typical applications which appear in transonic and supersonic flows over a strake-wing configuration at moderate-high angles of attack, at a supersonic inlet injesting a vortex and inside a supersonic combustor where fuel is injected in a swirling jet to enhance fuel-air mixing [1]-[3]. For the strake-wing configuration, vortex breakdown is undesirable since it results in the stall phenomenon, and hence its occurrence need to be delayed. On the other hand, vortex breakdown for the other two applications is desirable since it enhances mixing and stability of the flame [4]-[5], and hence its occurrence need to be controlled for the optimum performance. Unfortunately, the literature lacks this type of analysis with the exception of the preliminary work of Liu, Krause and Menne [6], Copening and Anderson [7], Delery, et al. [1], Kandil and Kandil [8] and Meadows, Kumer and Hussaini [9].

The first time-accurate NS solution for a supersonic vortex breakdown was developed by the present authors in Ref. [10]. We considered a supersonic quasi-axisymmetric vortex flow in a configured circular duct. The time-accurate solution of the unsteady, compressible NS equations was obtained using an implicit, upwind, flux-difference splitting finite-volume scheme. A shock wave has been generated near the duct inlet and unsteady vortex breakdown has been predicted behind the shock. The predicted flow was characterized by the evolution, convection merging and shedding of vortex breakdown bubbles. The Euler equations were 
also used to solve the same problem. The Euler solution showed larger size and number of vortex-breakdown bubbles in comparison with those of the NS solutions. The time-accurate solution was carried out for 3,200 times steps which are equivalent to a dimensionless time of 16. Only one value of Reynolds number of 10,000 was considered in Ref. [10].

In a later paper [11], we expanded our study of this flow using time-accurate computations of the NS equations with a fine grid in the shock-vortex interaction region and for longer computational times. Several issues were addressed in that study. First, we showed the effect of Reynolds number on the temporal evolution and persistence of vortex-breakdown bubbles behind the shock. In that stage of computations, the conditions at the downstream exit were obtained by extrapolating the components of the flowfield vector from the interior cell centers. Although the flow was supersonic over a large portion of the duct exit, subsonic flow existed over a small portion of the exit around the duct centerline. Therefore, selected flow cases were computed using a Riemann-invariant-type boundary conditions as well as other boundary conditions at subsonic points of the duct exit [12].

In the present paper, we consider shock/vortex interaction and the resulting vortex breakdown modes for quasi-axisymmetric and three-dimensional flows. This study covers bound and unbound flow domains. For the bound domain, supersonic swirling flow is considered in a configured duct, and for the unbound domain supersonic swirling flow that is issued from a nozzle into a uniform supersonic flow of lower Mach number is considered.

\section{Highlights of Formulation and Computational Scheme}

Formulation: The conservative, unsteady, compressible, full Navier-Stokes equations in terms of time-independent, body-conformed coordinates $\xi^{1}, \xi^{2}$ and $\xi^{3}$ are used to solve the problem. The equations are given in Ref. [11] and hence they are not presented here. Along with these equations, boundary conditions are specified at the computational-domain inlet, side wall and downstream exit. The downstream exit boundary conditions will be presented and discussed in the next section of the computational results. The initial conditions are also presented in the next section.

Computational Scheme: The computational scheme used to solve the unsteady, compressible full NS equations is an implicit, upwind, flux-difference splitting, finite-volume scheme. It employs the flux-difference splitting scheme of Roe which is based on the solution of the approximate one-dimensional Riemann problem in each of the three directions. In the Roe scheme, the inviscid flux difference at the interface of a computational cell is split into left and right flux differences. The splitting is accomplished according to the signs of the eigenvalues of the Roe averaged-Jacobian matrix of the inviscid flux at the cell interface. The smooth limiter is used to eliminate oscillations in the shock region. The viscous and heat-flux terms are differenced using second-order spatially accurate central differencing. The resulting 
difference equation is approximately factored and is solved in three sweeps in the $\xi^{1}, \xi^{2}$ and $\xi^{3}$ directions. The scheme is coded in the computer program which is called "FTNS3D".

The quasi-axisymmetric solutions are obtained using the three-dimensional code by forcing the flowfield vector to be equal on two axial planes, which are in close proximity of each other.

\section{Computational Results and Discussion}

a. Quasi-axisymmetric Vortex Breakdown Modes in a Configured Duct: Figure 1 shows an axial plane of a configured circular duct. The design of the duct configuration is intended to ensure that the supersonic inflow becomes supersonic at the exit. However, as the computation will show, a small portion of the duct exit becomes subsonic at certain times for the specified inflow conditions. A grid of $221 \times 51 \times 2$ in the axial, radial and circumferential directions, respectively, is used for the quasi-axisymmetric flow. For the three-dimensional flow, a grid of $221 \times 51 \times 49$ in the axial, radial and circumferential directions, respectively, is used. The minimum grid length is 0.002 . The upstream Mach number is kept at 1.75 .

The initial profile for the tangential velocity is given by

$$
\frac{w}{U_{\infty}}=\frac{k_{e}}{r}\left[1-\exp \left(-\frac{r^{2}}{r_{m}^{2}}\right)\right]
$$

where $U_{\infty}=1.74, r_{m}=0.2$ and $k_{e}=0.1$. The maximum $\frac{w}{U_{\infty}}$, swirl ratio $\beta$, is at $r=$ 0.224 and its value is kept at 0.32 . The radial velocity, $v$, at the initial station is set equal to zero and the radial momentum equation is integrated to obtain the initial pressure profile. Finally, the density $\rho$ is obtained from the definition of the speed of sound for the inlet flow. With these compatible set of profiles, the computations are carried out accurately in time with $\Delta t=0.0025$. The wall boundary conditions follow the typical Navier-Stokes solid-boundary conditions.

Reynolds number, $\operatorname{Re}=4,000$ : Figure 2 shows snapshots of the streamlines and Mach contours for the flow case of $\operatorname{Re}=4,000$. For this value of Reynolds number, a single breakdown bubble is seen at $t=5$ and it is convected downstreams as time passes. This breakdown bubble is formed during the downstream motion of the inlet shock, which reaches its maximum downstream displacement at $t=5$. Later on, the shock moves upstream, as it is seen at $\mathrm{t}=8$, while the breakdown bubble is convected in the downstream direction. Thereafter, the shock stays stationary at the inlet. This swirling flow case shows a transient single-bubble breakdown flow. The conditions at the exit are obtained by extrapolation from the interior cell centers.

Reynolds number, $R e=20,000$ : Figure 3 shows snapshots of the streamlines and Mach contours for this case. These snapshots show a vortex breakdown mechanism of evolution, 
convection, merging and shedding of bubbles while the inlet shock is moving downstreams, then upstreams and finally downstreams. The inlet shock becomes stationary and no more bubbles are developed. This swirling flow case shows a transient multi-bubble breakdown flow.

Reynolds number, $\operatorname{Re}=100,000$ : Figure 4 shows snapshots of the streamlines and Mach contours for this case. The downstream boundary conditions are obtained by extrapolating all the flow variables from the cell centers at the exit. The streamlines snapshots show multibubble vortex breakdown evolution, convection, merging and shedding. The time-accurate integration was carried out up to $t=200$ and the solution showed periodic multi-frequency cycles of vortex-breakdown bubbles [21]. An example of the merging of vortex breakdown bubbles of same sign of vorticity is shown at $t=17$. An example of convection and shedding of vortex breakdown bubbles is shown at $t=25$. Comparing the streamlines solutions at $t$ $=25$ and $\mathrm{t}=89$, it is seen that the solutions are almost the same which conclusively show that the breakdown process is periodic. The Mach-contours show the dynamics of inlet shock motion. In the time range of $t=3-8$, the inlet shock moves upstream toward the inlet and its central portion exists outside the inlet section at $t=8$. In the time range of $t=8-25$, the inlet shock moves downstream with corresponding evolution, convection, merging and shedding of breakdown bubbles. In the time range of $t=25-45$, the inlet shock maintains its motion in the downstream direction at a slower rate than before, while another shock, which is downstream of the inlet shock, appears and also moves in the downstream direction. The evolution, convection and shedding slowly continues until $t=66$. In the time range of $t=66$ 78, the downstream shock disappears and a large vortex-breakdown bubble appears and moves upstream. This motion of the bubble is accompanied by upstream motion of the inlet shock $(t=78)$. Later the inlet shock again moves in the downstream direction and the process is repeated. An animation movie has been produced for the total dimensionless time of $t=200$.

Exit Riemann Invariant Condition, $\mathrm{p}_{\mathbf{b}}=2 \mathrm{p}_{\infty}$ : In this case, the back pressure at the subsonic points of the duct exit is specified to be $p_{b}=2 p_{\infty}$ and the other four variables are extrapolated from the interior cell centers. Figure 5 shows snapshots of the streamlines and Mach contours of the solution. Comparing the present solution with the solution of the previous case (Fig. 4), it is seen that the two solutions are similar with the exception that the present solution lags that of the first case in time. The reason behind this behavior is that the back pressure $p_{b}$ is larger than that of the first case. Moreover, the Riemann invariant type conditions at subsonic points let the downstream effects propagate upstream as time increases. The existence of the large back pressure which is felt upstream supports the inlet shock and keeps it in the inlet region.

b. Three-Dimensional Vortex-Breakdown in a Configured Duct: Figure 6 shows the streamlines and Mach contours for the three-dimensional vortex breakdown modes in a con- 
figured duct with the same inflow conditions as those of Fig. 4. At the downstream exit, flow conditions are extrapolated from the interior cell centers. It is observed from the vertical, axial planes that the vortex breakdown is a three-dimensional phenomenon. The breakdown changes from a two-bubble type $(t=2-5)$ to a multi-bubble type $(t=7-34)$. At certain times, $t=11$ and 19 , it is observed that the multi-bubble breakdown is followed by a spiral breakdown. Beyond $t=46$, the breakdown becomes a single-bubble type.

c. Quasi-axisymmetric Vortex-Breakdown of a Supersonic Flow from a Nozzle: Here, a supersonic swirling jet at $M_{j}=3.0$, which is issued from a nozzle into a supersonic uniform flow of $M_{\infty}=2.0$, is considered. A grid of $221 \times 51 \times 2$ in the axial, radial and tangential directions, respectively is considered. The computational domain in an axial plane has the dimensions of $7.0 \times 3.5$ in the axial and radial directions, respectively, where the nozzle exit radius $r=1$. The freestream Reynolds number is 296,000 . The inflow profiles are taken from the experimental data of Ref. [2] and they are used as quasi-axisymmetric profiles [12]. Figure 7 shows snapshots of the streamlines and Mach contours at selected time steps. The streamline figures show multi-bubble breakdown at the early levels of time. These bubbles develop due to the shock system existing at the nozzle exit. The shock system consists of two oblique shocks; one is weak and the other is strong. For $\mathrm{t}>55$, the shock system and the breakdown bubble move upstreams and remain there with low frequency oscillation.

d. Three-Dimensional Vortex-Breakdown of a Supersonic Flow from a Nozzle: The flow of the previous case is considered for three-dimensional computations using a square-cross flow computational domain and a circular cross-flow computational domain. For the first case, one-half the square side is 3.5 units and for the second case, the radius is 3.5 units. Three types of grid are used for the first case (Gird types 1,2 and 3) and one type of grid is used for the second case (Grid type 4), see Fig. 8. This study shows the dominant effect of the grid shape and distribution on the vortex breakdown solutions. While grid type 1 shows two large breakidown bubbles, Grids 2, 3 and 4 show substantially smaller breakdown bubbles. Moreover, the conical shock system ahead of the breakdown bubbles shows substantial difference in size, location and shape. This study gives an alarming conclusion to the researchers who use substantially coarse grids with a rectangular cross-flow domain and a rectangular grid, and still claim capturing of physical vortex-breakdown flows. In the present study, the vortex breakdown bubbles of grid type 4 are of the same size order as those of grid type 3, although the shock system is somewhat different in shape.

\section{Concluding Remarks}

Computational simulation and study of shock/vortex interaction and vortex breakdown have been considered for internal and external supersonic swirling flow. The time-accurate computation for full Navier-Stokes equations is used to produce all the present cases. 
Numerous modes of vortex breakdown flows have been captured. The results show the effects of Reynolds number, downstream exit conditions, and grid shape, fineness and distribution. Three-dimensional vortex breakdown computations show somewhat different results from the quasi-axisymmetric counter parts since the three-dimensional flow provides a flow reliefing effect in the circumferential direction.

\section{Acknowledgement}

For the first two authors, this research work has been supported by NASA Langley Research Center under Grant No. NAG-1-994.

\section{References}

1. Delery, J.; Horowitz, E.; Leuchter, O.; Solignac, J. L.: Fundamental Studies of Vortex Flows. La Recherche Aérospatiale, No. 1984-2, 1984, pp. 1-24.

2. Metwally, O.; Settles, G.; Horstman, C.: An Experimentally Study of Shock Wave/Vortex Interaction. AIAA 89-0082, January 1989.

3. Cutler, A. D.; Levey, B. S.: Vortex Breakdown in a Supersonic Jet. AIAA 91-1815, June 1991.

4: Rhode, D. L.; Lilley, D. G.; McLaughlin, D. K.: On the Prediction of Swirling Flowfields Found in Axisymmetric Combustor Geometries. Transactions of ASME, Vol. 104, September 1982, pp. 378-384.

5. Altegeld, H.; Jones, W. P.; Wilhelmi: Velocity Measurements in a Confined Swirl Driven Recirculating Flow. Experiments in Fluids, Springer Verlag, Vol. 1, 1983, pp. 73-78.

6. Liu, C. H.; Krause, E.; Menne, S.: Admissible Upstream Conditions for Slender Compressible Vortices. AIAA 86-1093, 1986.

7. Copening, G.; Anderson, J.: Numerical Solutions to Three-Dimensional Shock/Vortex Interaction at Hypersonic Speeds. AIAA 89-0674, January 1989.

8. Kandil, O. A.; Kandil, H. A.: Computation of Compressible Quasi-Axisymmetric Slender Vortex Flow and Breakdown. IMACS 1st International Conference on Computational Physics, University of Colorado, Boulder, June 1990, pp. 46-51. Also in Computer Physics Communications, Vol. 65, North-Holland, March 1991, pp. 164-172.

9. Meadows, K.; Kumar, A.; Hussaini, M.: A Computational Study on the Interaction Between a Vortex and a Shock Wave. AIAA 89-1043, April 1989.

10. Kandil, O. A.; Kandil, H. A.; Liu, C. H.: Computation of Steady and Unsteady Compressible Quasi-Axisymmetric Vortex Flow and Breakdown. AIAA 91-0752, January 1991.

11. Kandil, O. A.; Kandil, H. A.; Liu, C. H.: Supersonic Quasi-Axisymmetric Vortex Breakdown. AIAA 91-3311-CP, September 1991, pp. 851-863.

12. Kandil, O. A.; Kandil, H. A.; Liu, C. H.: Critical Effects of Downstream Boundary Conditions on Vortex Breakdown. ALAA 92-2601-CP, June 1992. 


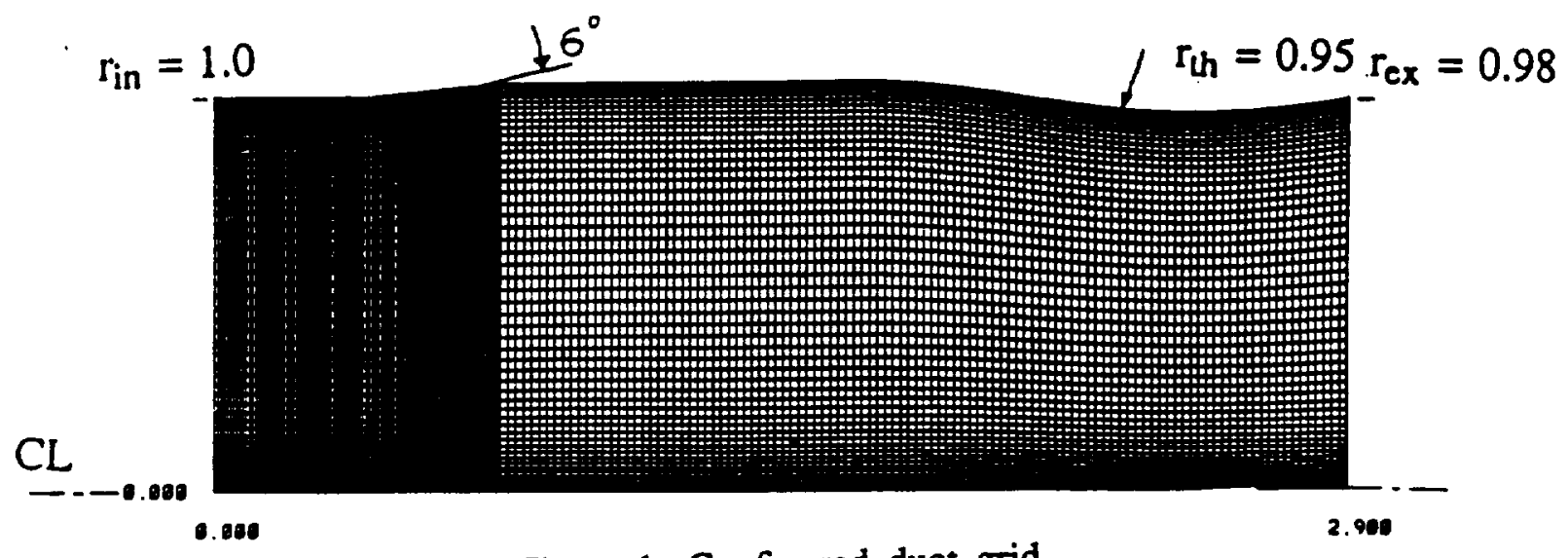

Figure 1. Configured duct grid
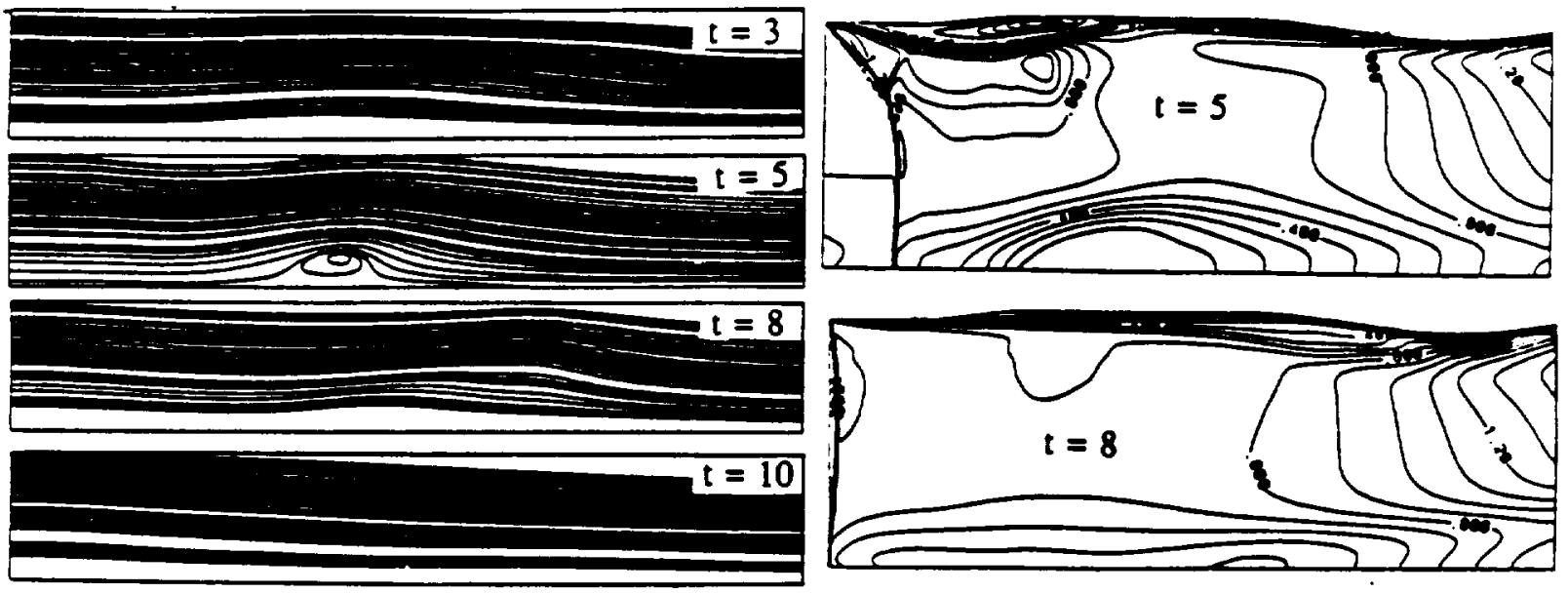

Figure 2. Streamlines and Mach contours for a swirling flow with a transient single-bubble breakdown, $\mathrm{M}_{\infty}=1.75, \beta=0.32, \mathrm{Re}=4,000$.
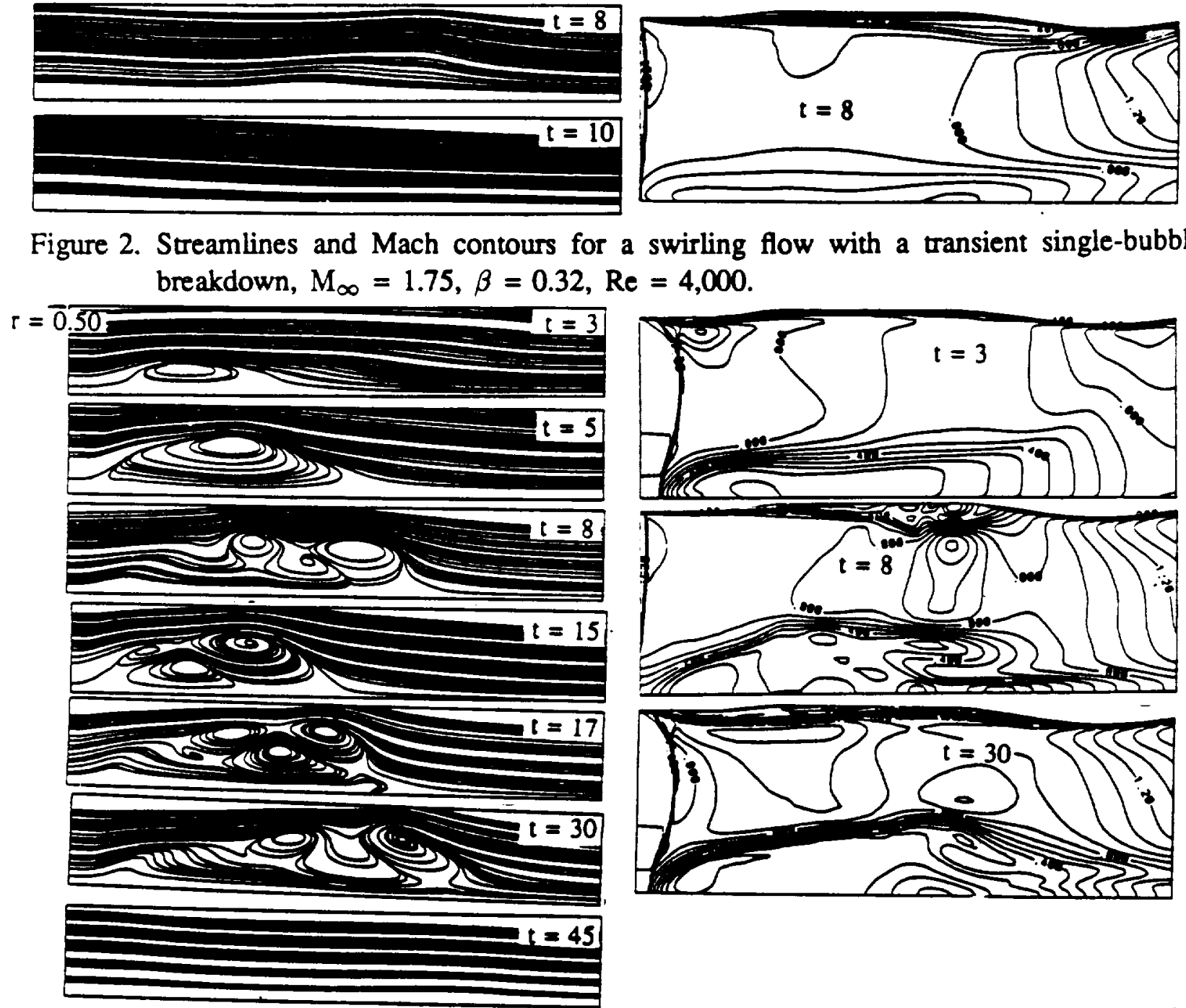

Figure 3. Streamlines for a swirling flow with transient multi-bubble breakdown, $\mathrm{M}_{\infty}=1.75$, $\beta=0.32, \operatorname{Re}=20,000$
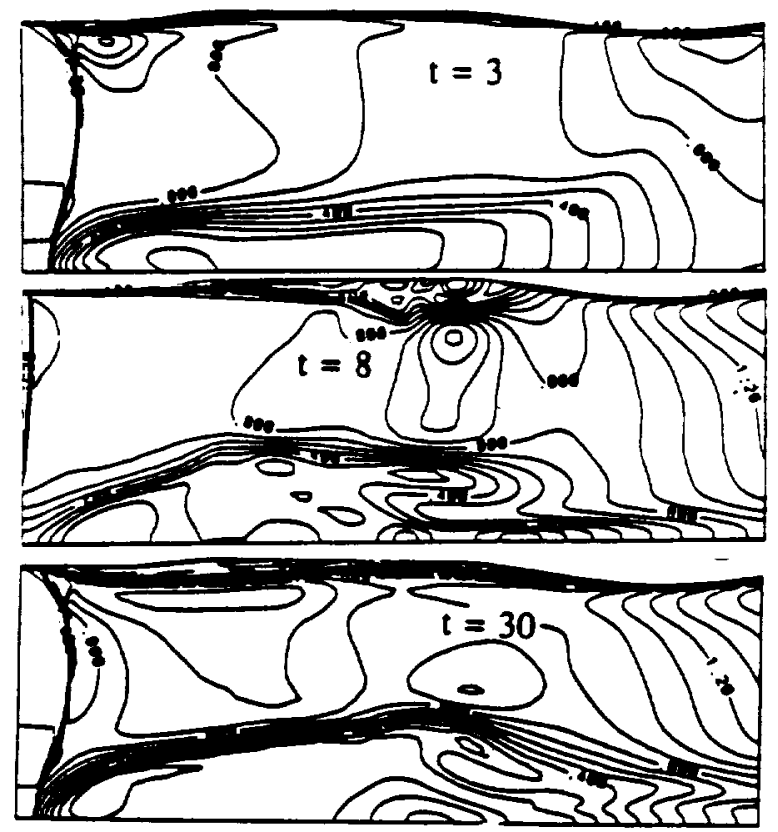

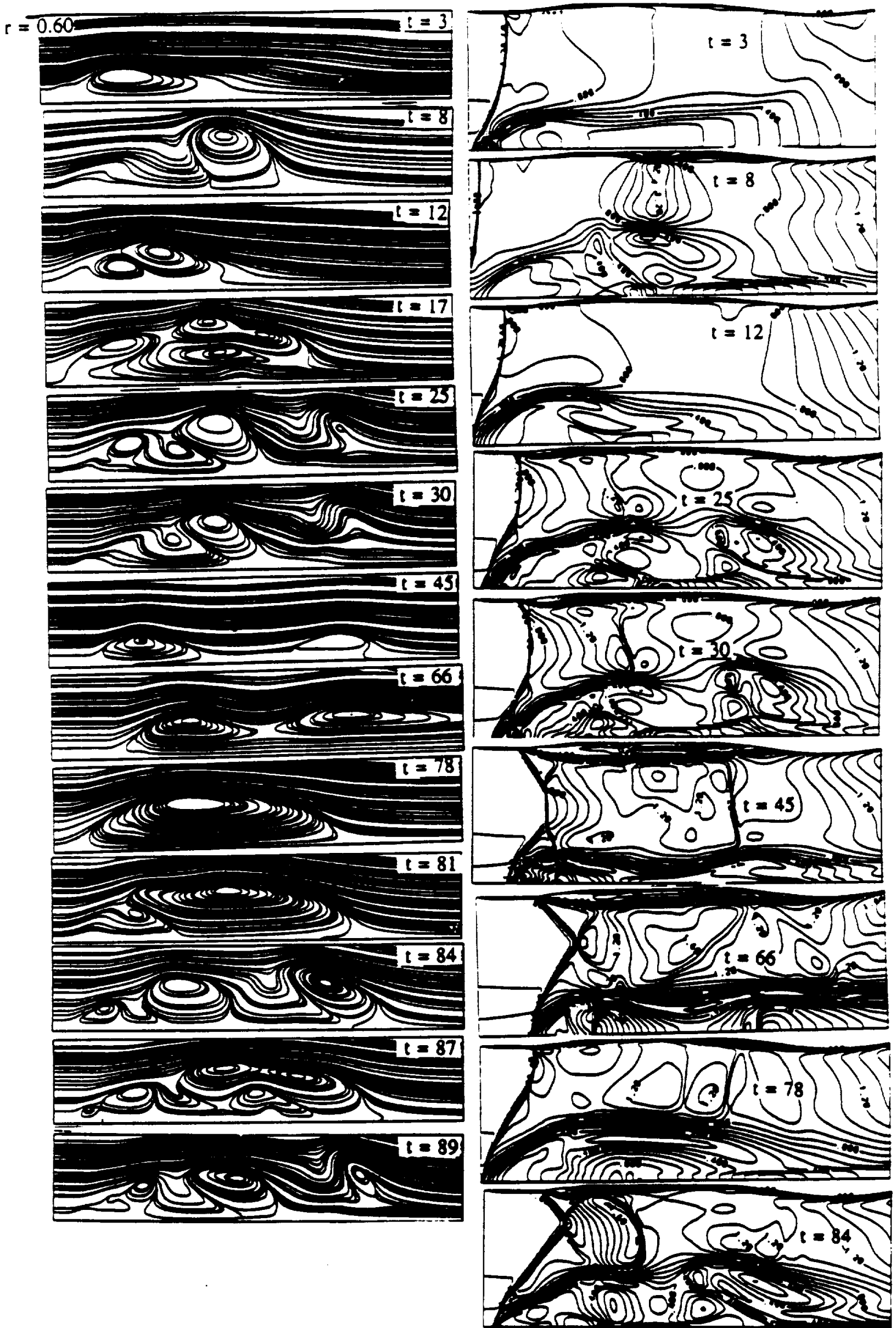

Figure 4. Streamlines and Mach contours for a swirling flow with periodic multibubble, multifrequency vortex breakdown, extrapolation from interior 

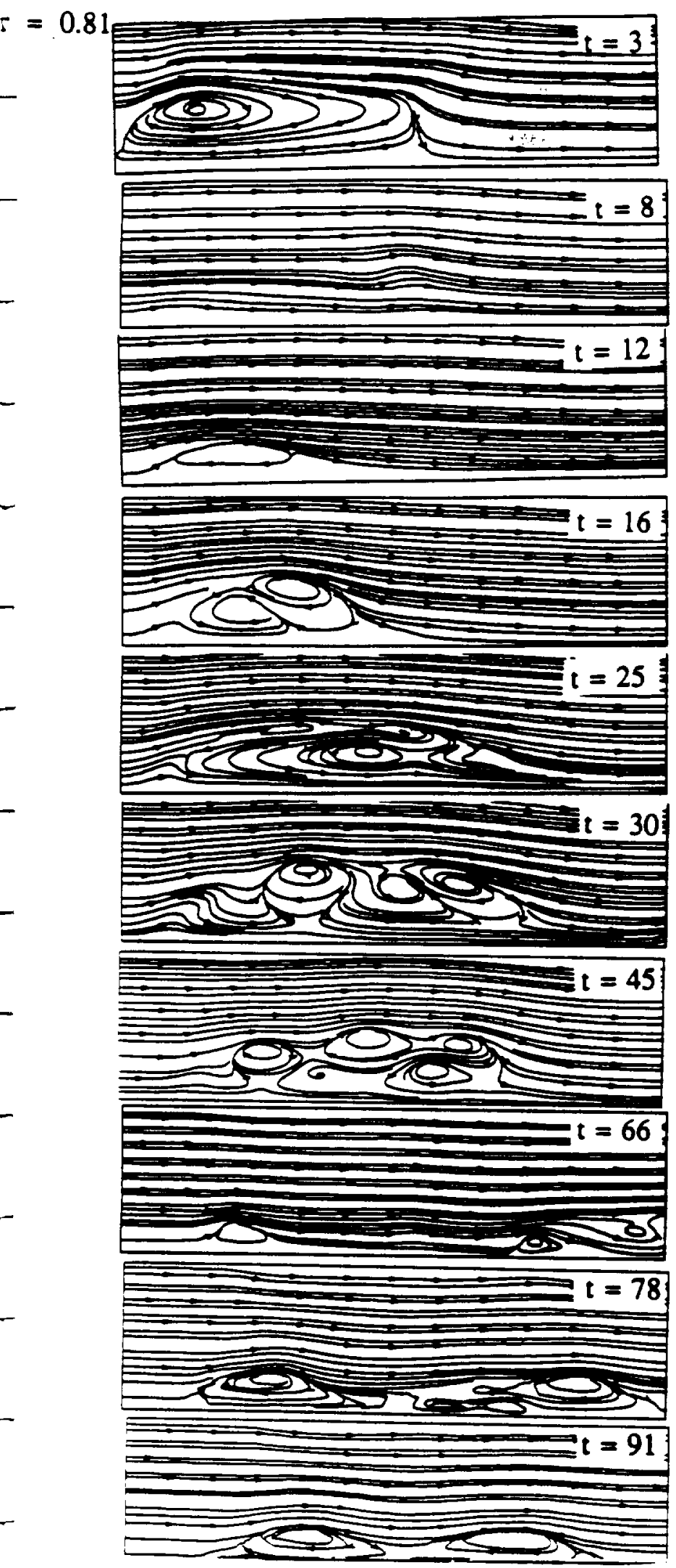
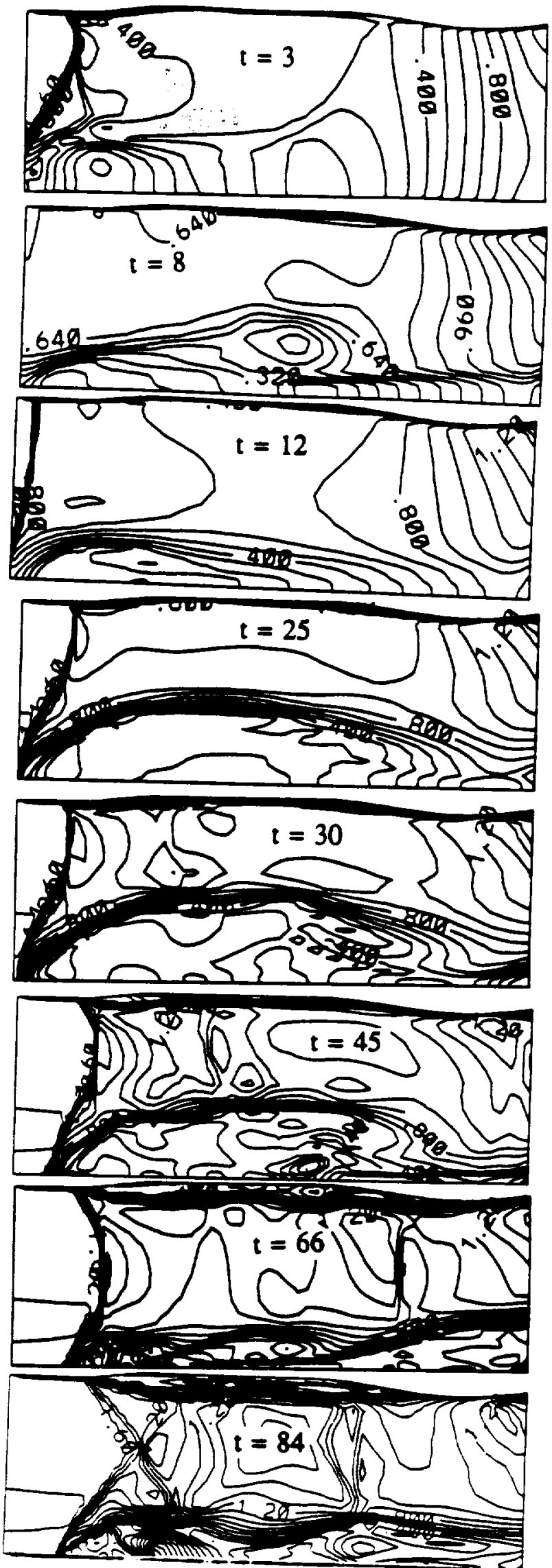

Figure 5. Streamlines and Mach contours for a swirling flow with periodic multibubble, multifrequency vortex breakdown, $\mathrm{P}_{\mathrm{b}}=2 \mathrm{P}_{\infty}$, Riemann invariant B.C. 

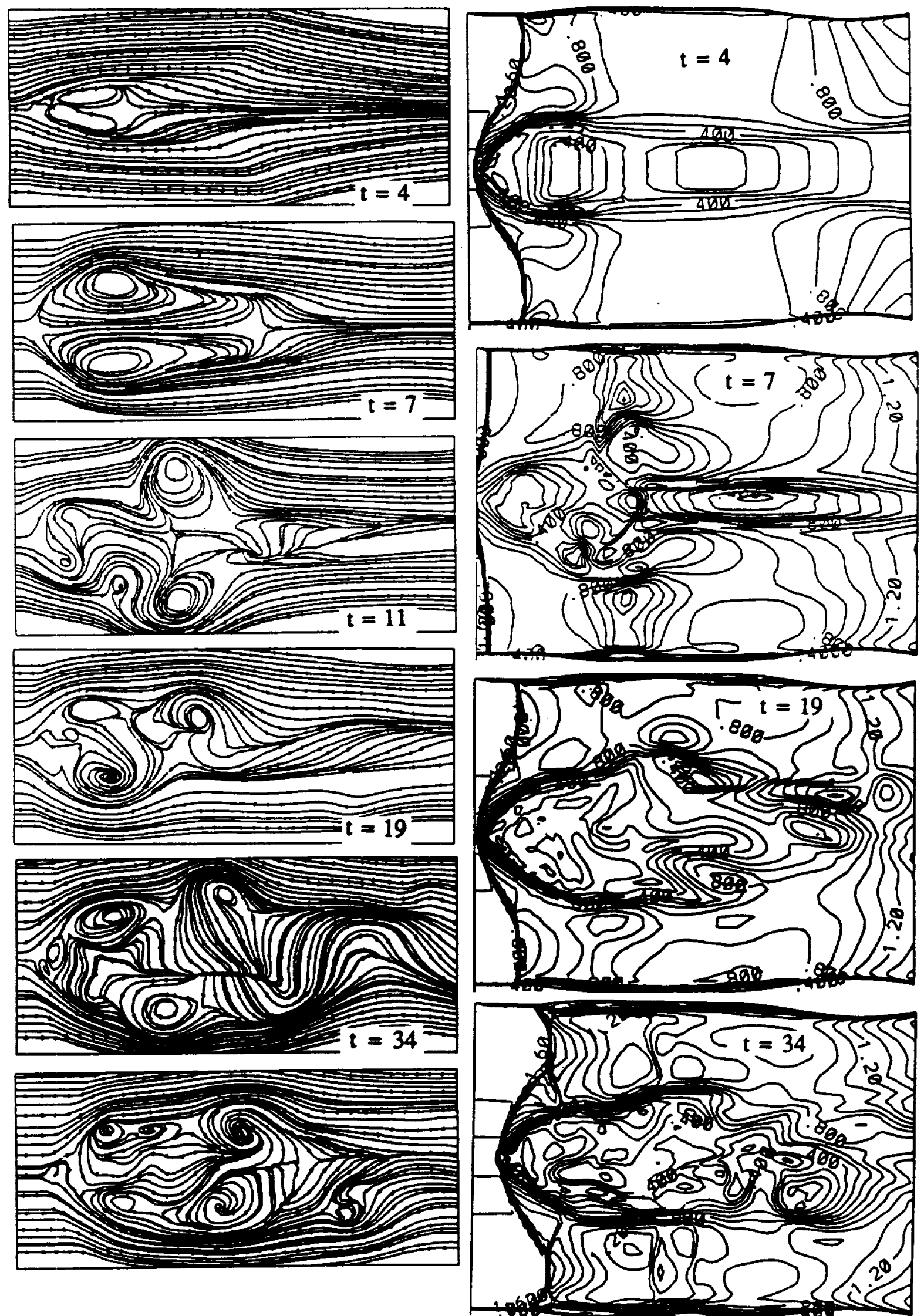

Figure 6. Mach contours and streamlines for a swirling three-dimensional flow in a configured circular duct, $\mathrm{M}_{\infty}=1.75, \operatorname{Re}=100,000, \beta=0.32$ 
$r=0.695$
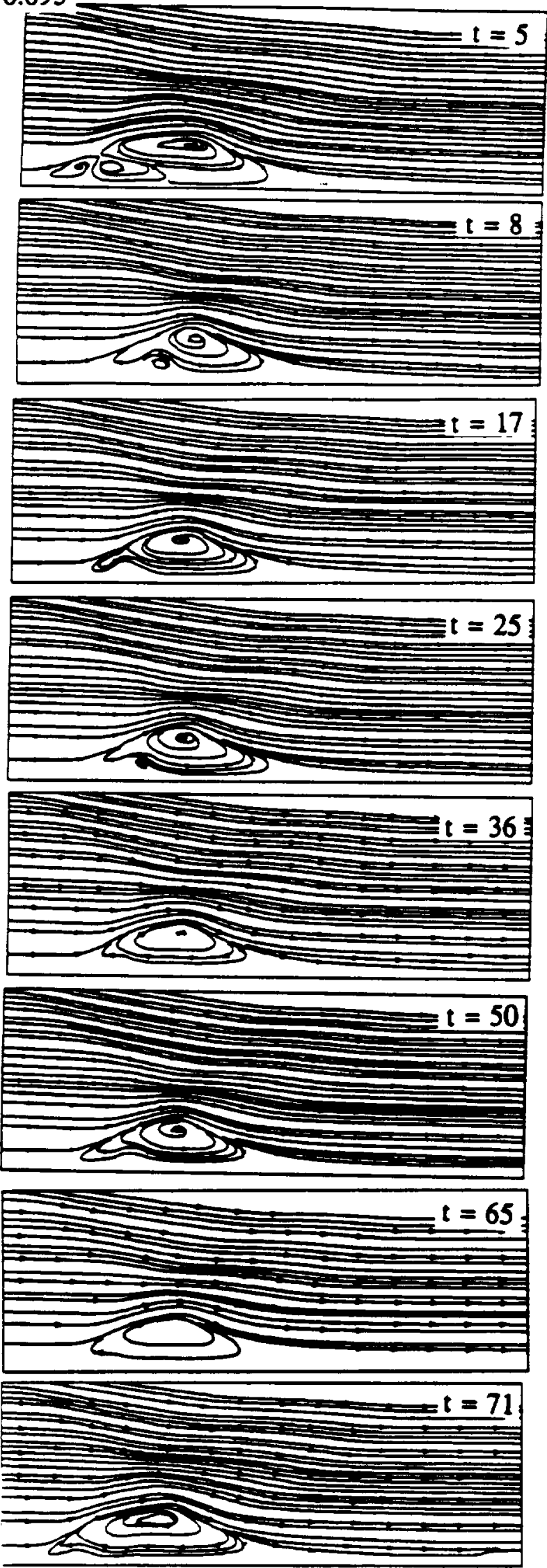

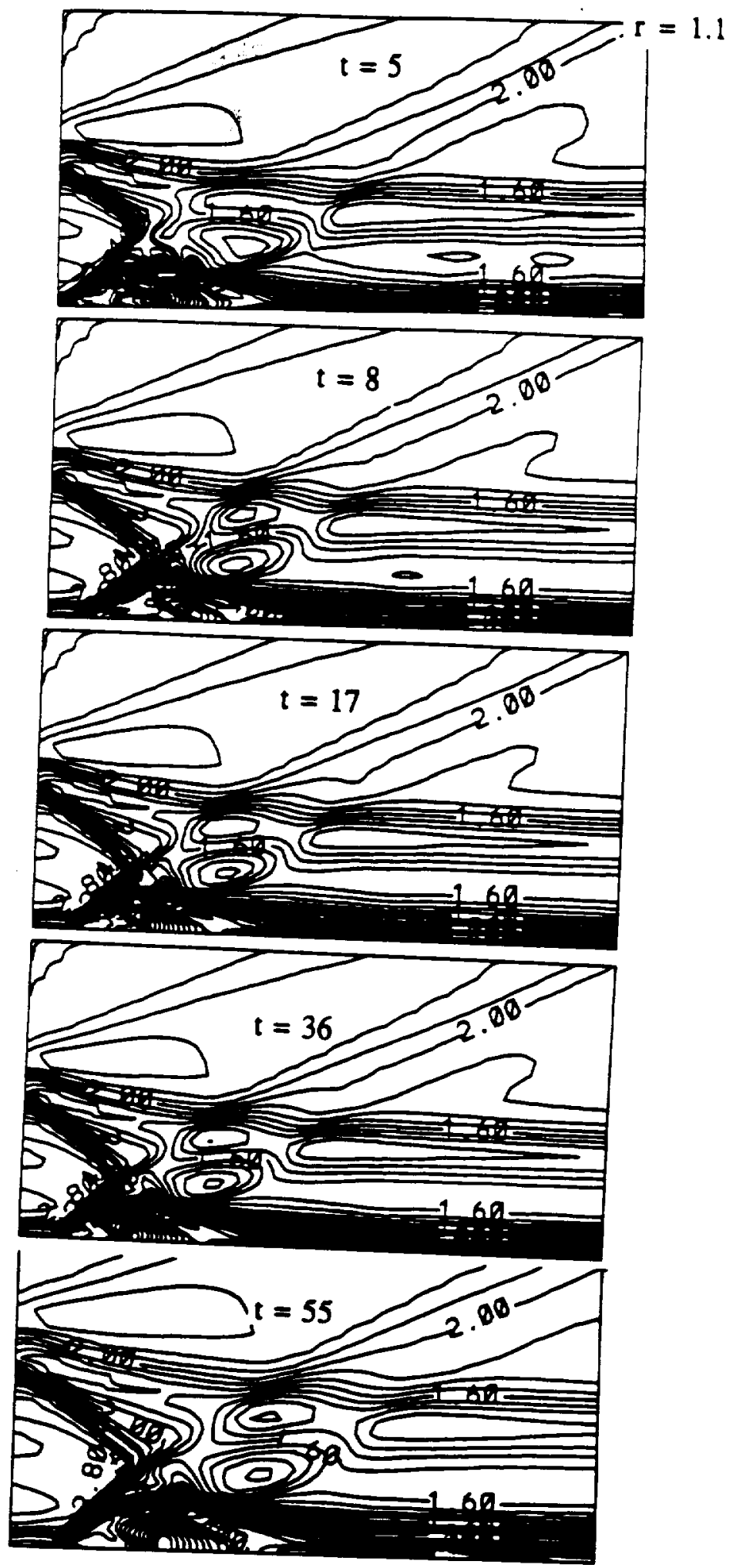

Figure 7. Streamlines and Mach contours for supersonic swirling jet from a nozzle with lowfrequency almost single bubble vortex breakdown, extrapolation from interior B.C. 


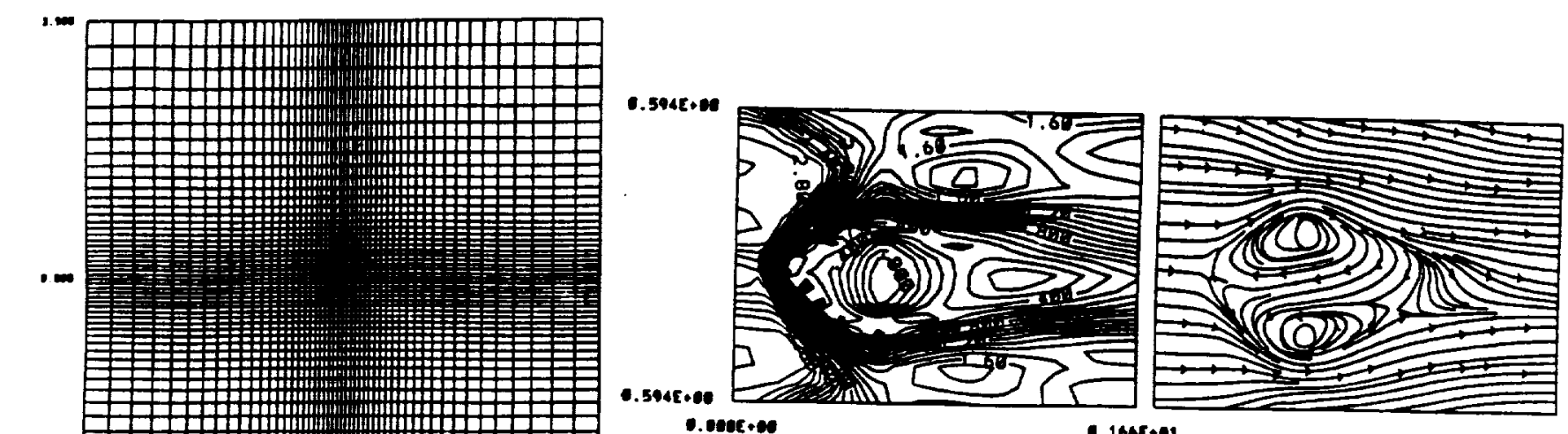

-.ease +es

Grid type 1, cross-flow grid $51 \times 51,221$ in axial direction.

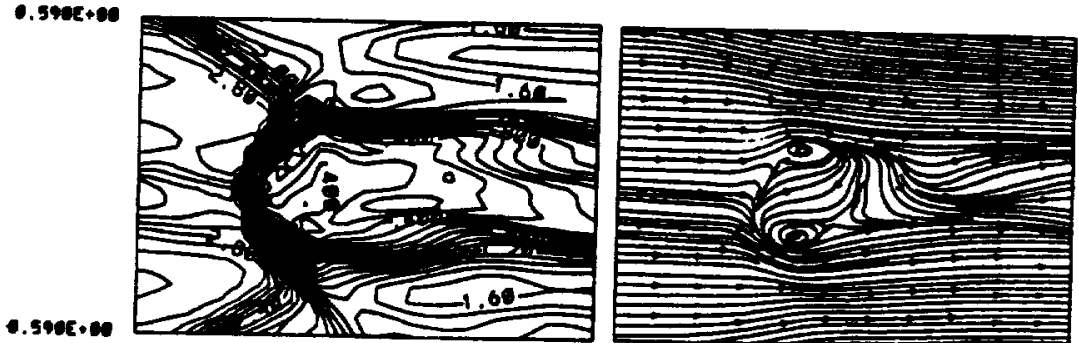

c.esceres

$0.17 k+01$

Grid type 2, cross-flow frid $61 \times 61,145$ in axil direction.

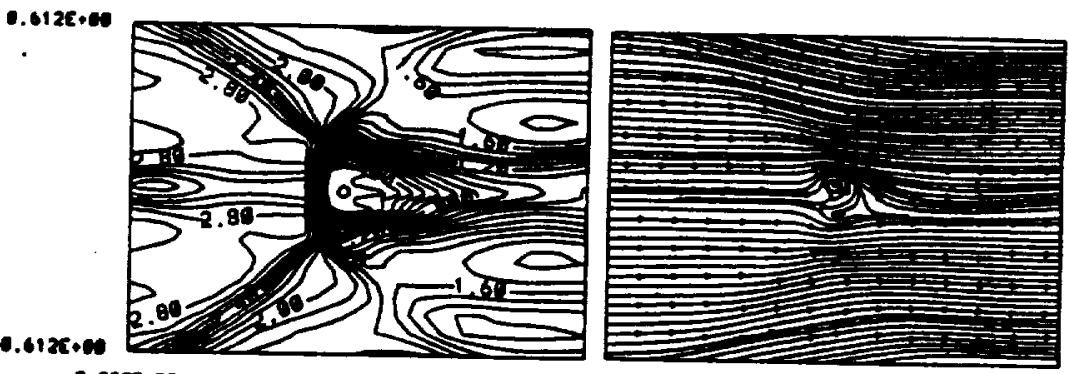

e.enceses $0.16 x+91$

Grid type 3, cross-flow frid $61 \times 61,145$ in axial direction.
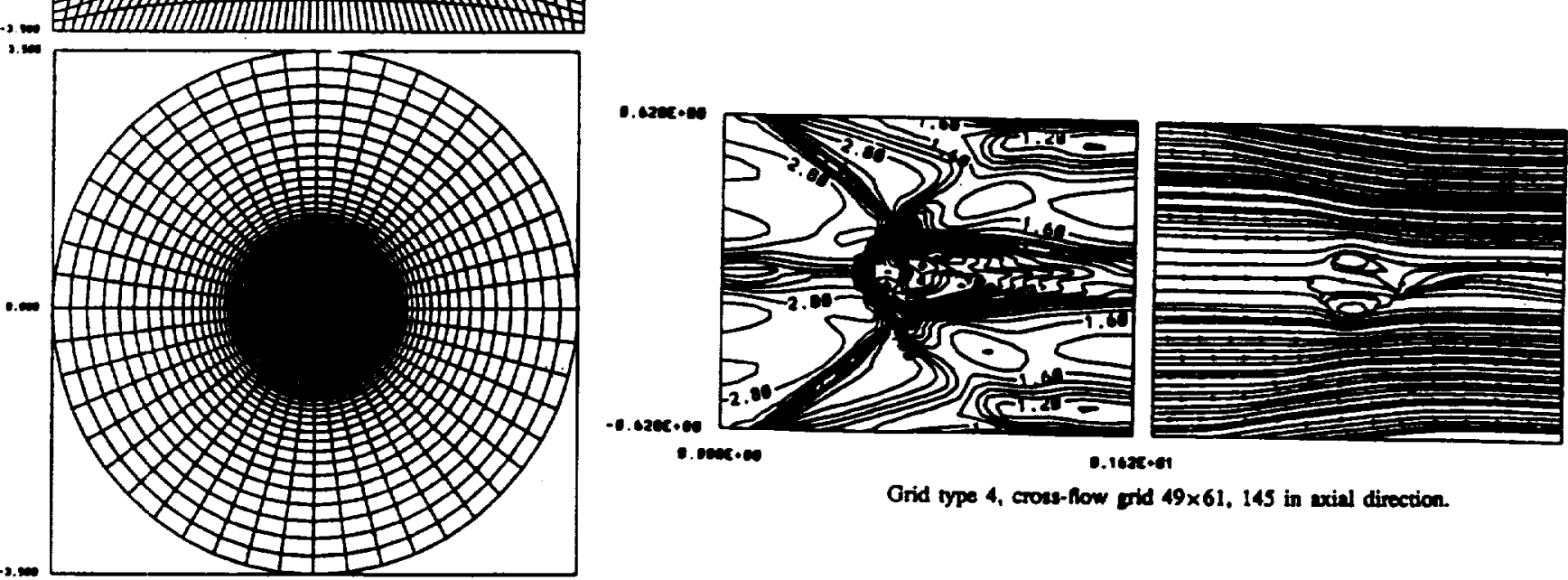

c. onesece

$0.145+4$

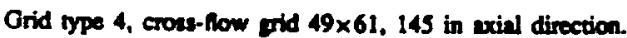

Figure 8. Mach contours and streamlines for vortex breakdown in a vertical axial plane for a supersonic swirling for from a nozzle, $t=10, M_{j}=3.0, M_{\infty}=2.0, R e=296,000$ 


\title{
三
}

\author{
AIAA-92-4429-CP
}

\section{COMPUTATION OF VORTEX WAKE FLOWS AND CONTROL OF THEIR EFFECTS ON TRAILING WINGS}

Tin-Chee Wong and Osama A. Kandil

Old Dominion University, Norfolk, VA 23529

C. H. Liu

NASA Langley Research Center, Hampton, VA 23665

\section{AIAA Atmospheric Flight Mechanics Conference Hilton Head, SC, August 10-12, 1992}





\title{
COMPUTATION OF VORTEX WAKE FLOWS AND CONTROL OF THEIR EFFECTS ON TRAIING WINGS
}

\author{
Tin-Chee Wong" and Osama A. Kandil"* \\ Old Dominion University, Norfolk, VA 23529 \\ and \\ C.H. Liu \\ NASA Langley Research Center, Bampton VA 23665
}

\begin{abstract}
The near-vortex-wake flow of a lange aspect-ratio rectangular wing is accurately computed by using the thinlayer and full Navier-Stokes (NS) equations. The chordwise section of the wing is a NACA-0012 airfoil and its tip is round. The computations have been carried out on a fine C-O grid using an implicit, upwind, flux-difference splitting, finite-volume scheme. The thin-layer NS results have been obtained with and without flux limiters and the full NS results have been obtained without flux limiters. Flow transition from laminar to turbulent is mimiced by turning-on the Baldwin-Lomax algebraic model at an experimentally prescribed chord-station location of 0.05 . Comparison of the computed results with each other and with the experimental data shows that the full NS results give the best resolution of the near-vortex-wake flow. Next, the strength of the wing-tip vortex has been reduced substantially without reducing the lift coefficient by using flow-injection from a slot along a portion of the wing tip. The flow injection is directed in the wing plane at $45^{\circ}$ with the wing-tip chord.
\end{abstract}

\section{INTRODUCTION}

Recently, there has been an alarming rate of increase of the volume of air civil transport at many airports. With this status of air traffic congestion, the hazardous effect of wake vortices emanating from large aircraft on light aircraft, which pass through these vortices during landing and take-off operations, is becoming a vital concern for operations safety. These vortices are characterized with high intensity and turbulence, and may produce high rolling moments on trailing aircraft which could exceed the available roll control of the trailing aircraft. Moreover, the trailing aircraft, under the influence of these vortex trails, could suffer a loss of altitude or climb rate in addition to structural damages. These vortices may persist up to several miles and for long periods of time before their decay, and therefore they play a major role in sequencing landing and take-off operations at busy airports. For example, a minimum safe-separation distance between aircraft is dictated depending on the vortex intensity, wind shear, atmospheric turbulence and temperature gradient; among others.

\footnotetext{
Research Associate, Dept. of Mechanical Engineering and Mechanics, Member ALAA.

-- Professor and Eminent Scholar, Dept of Mechenical Engineering and Mechanics, Associate Fellow ALAA.

-.. Group Leader, Theoretical Flow Physics Branch, Associare Fellow AIAA.
}

The literature shows a few experimental and computational investigations that attempt to model and analyze wake vortex interaction, merging, decay and their hazardous effects on trailing aircraft. Hallock and Eberle ${ }^{1}$ presented a review of the state of the ant of aircraft wake vortices covering the research efforts in the United States until the mid-seventies. Experimental wind tunnel and airport measurements of the vortex wakes were conducted by Dee and Nicholas ${ }^{2}$ Harvey and Perry ${ }^{3}$, El-Ramly ${ }^{4}$, Wood and McWilliams ${ }^{5}$, Gardoz ${ }^{6}$, Cliffone and Lonzo', Olwi and Ghazi', Liu' and Liu et al $^{10}$.

Mathematical models and computational schemes were developed using inviscid analysis by Chorin and Bernard ${ }^{11}$. Hacket and Evans ${ }^{12}$. Yates ${ }^{13}$. Iversen and Bernstein $^{\text {i4 }}$, and Rossow ${ }^{15}$. Although an inviscid model cannot describe the wake aging including its diffusion, it is still capable of producing the wake shape and its dynamics. The mathematical modeling used in the above references were based on the use of the point vortex method to compute the motion of a finite number of point vortices which model the vorticity behind a wing. The first three-dimensional inviscid model was introduced by Kandil, et. $\mathrm{al}^{16}$, where the nonlinear vortex-lattice, which was also developed by Kandil, et. al ${ }^{17}$. was used to compute the interference flow between wings and the vortex wake hazardous effects.

Viscous modeling of trailing vortices was first introduced by McCormick, et $a^{18}$. Viscous interactions in vortex wakes and the effects of background turbulence, wind shear and ground on two-dimensional vortex pairs were presented by Bilanin, et al $\mathbf{a l}^{19}, 20$. Liu and his co-workers $\mathrm{s}^{21-24}$ studied the interaction, merging and decay of vortices in two-dimensional space and of threedimensional vortex filaments. To estimate the effects of density stratification, turbulence and Reynolds number on vortex wakes, an approximate model was recently developed by Greene ${ }^{25}$. Later on, Greene and his co-workers ${ }^{26}$ presented selected results of aircraft vortices which include a juncture vortex, a lifting-wing vortex and a wake vortex.

It is concluded from the above brief literature survey that the problem contains several vortex flow regions along with several critical parameters influencing the vortex wake flows. The vortex wake flow of interacting-wing problems can be divided into three regions. The first region includes the leading wing and its near-vortex-wake flow. The second region includes the interacting vortices in the wake. The third region includes the far-wake flow along with the trailing-wing flow. In Ref. 27, Kandil, 
Wong and Liu presented results of the near-vortex-wake flow for a large-aspect-ratio rectangular wing using the thin-layer, Reynolds averaged, NS equations on a C-O grid. Next, a small aspect-ratio rectangular wing was introduced in the near-wake of the leading wing and the problem was recomputed to demonstrate the hazardous effects of the vortex wake flow. Two interference cases were considered. The first was called the "along-track penetration through vortex center" and the second was called the "along-track penetration between vortices", see Fig. 1. It was demonstrated that in the first case, the trailing wing experienced large rolling moments and in the second case the trailing wing experienced loss of lift.

In the present paper, the near-vortex-wake flow is recomputed using a finer grid than the grid used in Ref. 27. Moreover, the thin-layer and full NS equations are used along with an upwind scheme, wherein the flux limiter of the solver is tumed on and off. The goal of this part of the paper is to accurately compute the vortex wake flow and to study the effect of the numerical dissipation of the flux limiter on the computed results. In the second part of the paper, alleviation of the strength of the tip vortex without reducing the lift force is demonstrated by using directed flow injection from a slot along a certain length of the wing tip.

\section{FORMULATION}

\section{Full and Thin-Layer Navier-Stokes Equations}

The conservative form of the dimensionless, unsteady, compressible, full Navier-Stokes equations in terms of time-independent, body-conformed coordinates $\xi^{1}, \xi^{2}$ and $\xi^{3}$ is given by

$$
\frac{\partial \overline{\mathrm{Q}}}{\partial \mathrm{t}}+\frac{\partial \overline{\mathrm{E}}_{\mathrm{m}}}{\partial \xi^{\mathrm{m}}}-\frac{\partial\left(\overline{\mathrm{E}}_{\mathrm{v}}\right)_{s}}{\partial \xi^{\mathrm{s}}}=0 ; \mathrm{m}=1-3, \mathrm{~s}=1-3
$$

where

$$
\begin{gathered}
\xi^{\mathrm{m}}=\xi^{\mathrm{m}}\left(\mathrm{x}_{1}, \mathrm{x}_{2}, \mathrm{x}_{3}\right) \\
\because \cdot \\
\overline{\mathrm{Q}}=\frac{\hat{\mathrm{q}}}{\mathrm{J}}=\frac{1}{\mathrm{~J}}\left[\rho, \rho \mathrm{u}_{\mathrm{t}}, \rho \mathrm{u}_{2}, \rho \mathrm{u}_{3}, \rho \mathrm{e}\right]^{\mathrm{t}}
\end{gathered}
$$

$$
\begin{aligned}
\overline{\mathrm{E}}_{\mathrm{m}} & \equiv \text { inviscid flux } \\
& =\frac{1}{\mathrm{~J}}\left[\partial_{\mathrm{k}} \xi^{\mathrm{m}} \hat{\mathrm{E}}_{\mathrm{k}}\right]^{\mathrm{t}} \\
& =\frac{1}{\mathrm{~J}}\left[\rho \mathrm{U}_{\mathrm{m}}, \rho \mathrm{u}_{1} \mathrm{U}_{\mathrm{m}}+\partial_{1} \xi^{\mathrm{m}} \mathrm{p}, \rho \mathrm{u}_{2} \mathrm{U}_{\mathrm{m}}\right. \\
& \left.+\partial_{2} \xi^{\mathrm{m}} \mathrm{p}, \rho \mathrm{u}_{3} \mathrm{U}_{\mathrm{m}}+\partial_{3} \xi^{\mathrm{m}} \mathrm{p},(\rho \mathrm{e}+\mathrm{p}) \mathrm{U}_{\mathrm{m}}\right]^{\mathrm{t}}
\end{aligned}
$$

$$
\begin{aligned}
& \left(\bar{E}_{v}\right)_{8} \equiv \text { viscous and heat - conduction flux in } \xi^{\circ}
\end{aligned}
$$

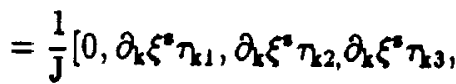

$$
\begin{aligned}
& \left.\partial_{k} \xi^{*}\left(u_{n} \lambda_{k n}-q_{k}\right)\right]^{\imath} ; \quad k=1-3, n=1-3
\end{aligned}
$$

$$
\mathrm{U}_{\mathrm{m}}=\partial_{\mathrm{k}} \xi^{\mathrm{m}} \mathbf{u}_{\mathrm{k}}
$$

The three momentum elements of Eq. (5) are given by

$$
\begin{aligned}
& \partial_{k} \xi^{\bullet} \lambda_{j} \equiv \frac{M_{\infty} \mu}{R e}\left[\left(\partial_{k} \xi^{a} \partial_{j} \xi^{n}-\frac{2}{3} \partial_{j} \xi^{a} \partial_{k} \xi^{a}\right) \frac{\partial u_{k}}{\partial \xi^{n}}\right. \\
& +\partial_{k} \xi^{2} \partial_{k} \xi^{n} \frac{\partial u_{j}}{\partial \xi^{*}} ; j=1-3
\end{aligned}
$$

The last element of Eq. (5) is given by

$$
\begin{aligned}
& \partial_{k} \xi^{*}\left(u_{p} \lambda_{\mathrm{zp}}-q_{k}\right) \equiv \frac{\mathbf{M}_{\propto} \mu}{R e}\left[\left(\partial_{k} \xi^{*} \partial_{p} \xi^{n}\right.\right. \\
& \left.-\frac{2}{3} \partial_{\mathrm{p}} \xi^{\mathrm{s}} \partial_{\mathrm{k}} \xi^{\mathrm{n}}\right) \mathrm{u}_{\mathrm{p}} \frac{\partial \mathrm{u}_{\mathrm{k}}}{\partial \xi^{\mathrm{n}}} \\
& +\partial_{k} \xi^{*} \partial_{k} \xi^{n} u_{p} \frac{\partial u_{p}}{\partial \xi^{n}} \\
& \left.+\frac{1}{(\gamma-1) P_{z}} \partial_{1} \frac{\partial\left(a^{2}\right)}{\partial \xi^{n}}\right] ; p=1-3
\end{aligned}
$$

The single thin-layer approximations of the full NavierStokes equations demand that we only keep the derivatives in the normal direction to the body, $\xi^{2}$, in the viscous and heat fux terms in Eqs. (1), (7) and (8). Thus, we let $s=2$ for the term $\frac{\partial\left(E_{r}\right)}{\partial t}$ in Eq. (1) and $s=2$ and $n=2$ in Eqs. (7) and (8). These equations reduce to

$$
\begin{aligned}
& \frac{\partial \overline{\mathbf{Q}}}{\partial t}+\frac{\partial \overline{\mathrm{E}}_{\mathrm{m}}}{\partial \xi^{\mathrm{m}}}-\frac{\partial\left(\overline{\mathrm{E}}_{\mathrm{v}}\right)_{2}}{\partial \xi^{2}}=0 \\
& \partial_{k} \xi^{2} n_{k \perp} \equiv \frac{M_{\propto} \mu}{R e}\left(\psi \partial_{1} \xi^{2}+\phi \frac{\partial{u_{1}}_{1}}{\partial \xi^{2}}\right) \\
& \partial_{k} \xi^{2}\left(u_{p} \eta_{p p}-q_{k}\right) \equiv \frac{M_{\propto x} \mu}{R e}\{\psi W \\
& +\phi\left[\frac{1}{2} \frac{\partial}{\partial \xi^{2}}\left(u_{1}^{2}+u_{2}^{2}+u_{3}^{2}\right)\right. \\
& \left.\left.+\frac{1}{(\gamma-1) P_{r}} \frac{\partial\left(a^{2}\right)}{\partial \xi^{2}}\right]\right\}
\end{aligned}
$$

where

$$
\phi_{1}=\partial_{k} \xi^{2} \partial_{L} \xi^{2}, \psi=\frac{1}{3} \partial_{k} \xi^{2} \frac{\partial u_{k}}{\partial \xi^{2}}, W=\partial_{p} \xi^{2} u_{p}
$$

In Eqs. (1)-(12), the dimensionless variables are referenced to their appropriate freestream values. The dimensionless density $\rho$, cartesian velocity components $v_{1}, v_{2}$ and $u_{3}$, total energy per unit mass, e, dynamic viscosity, $\mu$ and speed of sound, a, are defined as the ratio of the corresponding physical quantities to those of the freestream; namely, $\rho_{\infty}, a_{\infty}, \rho_{\infty} a_{\infty}^{2}, \mu_{\infty}$ and $a_{\infty}$; respectively. The pressure, $p$, is non-dimensionalized by $\rho_{\infty} a_{\infty}^{2}$, and is related to the lotal energy for a perfect gas by the equation of state

$$
p=(\gamma-1) \rho\left(e-\frac{1}{2} u_{j} u_{j}\right) ; j=1-3
$$


where $\gamma$ is the ratio of specific heats and its value is 1.4. The viscosity, $\mu$, is calculated from the Sutherland law

$$
\mu=\mathrm{T}^{3 / 2}\left(\frac{1+\mathrm{c}}{\mathrm{T}+\mathrm{c}}\right), \mathrm{c}=0.4317
$$

where $T$ is the temperature which is non-dimensionalized by $\mathrm{T}_{x}$. The Prandul number, $\mathrm{P}_{\mathrm{f}}$ is fixed at 0.72 . The Reynolds number is defined as $\mathrm{R}_{e}=\rho_{\infty} \mathrm{U} \times \mathrm{L} / \mu_{\infty}$ and the characteristic length, $L$, is chosen as the root chord of the wing.

In Eqs. (1)-(13), the indicial notation is used for convenience. The subscripts $k, n, p$ and $j$ are summation indices, the superscript or subscript $s$ is a summation index and the superscript or subscript $m$ is a free index. The partial derivative $\frac{\partial}{\partial x_{k}}$ is referred to by $\partial_{k}$.

\section{Turbulent Flow}

For the turbulent flow, the Navier-Stokes equations are transformed to the Reynolds-averaged equations by replacing the coefficient of molecular viscosity, $\mu$, and the coefficient of thermal conductivity $k$ with

$$
\begin{gathered}
\mu_{\mathrm{t}}=\mu+\mu_{\mathrm{t}}=\mu\left(1+\mu_{\mathrm{t}} / \mu\right) \\
\mathrm{k}_{\mathrm{e}}=\mathrm{k}+\mathrm{k}_{\mathrm{t}}=\frac{\mu \mathrm{C}_{\mathrm{P}}}{\mathrm{P}_{\mathrm{I}}}\left(1+\frac{\mu_{\mathrm{t}}}{\mu} \frac{\mathrm{P}_{\mathrm{r}}}{\mathrm{P}_{\mathrm{r}}}\right)
\end{gathered}
$$

where $\mu_{e}$ is the effective viscosity, $k_{e}$ the effective thermal conductivity, $\mu_{r}$ the turbulent viscosity, $P_{r}$ the laminar Prandtl number, $P_{n}$ the turbulent Prandtl number and $C_{p}$ the specific heat under constant pressure. The urbulent viscosity $\mu_{\mathrm{t}}$ is obtained by using the two-layer algebraic eddy viscosity model which was first developed by $\mathrm{Cebeci}^{28}$ for the boundary-layer equations and modified later by Baldwin and Lomax ${ }^{29}$ for the Navier-Stokes equations. The details of the turbulent model is given in Ref. (30) by Wong, Kandil and Liu.

\section{Boundary and Initial Conditions}

Boundary conditions are explicitly implemented. They include the inflow-outflow conditions and the solid-boundary conditions. At the plane of geometric symmetry, periodic conditions are used. The inflowoutfow boundary conditions are implemented using the one-dimensional Riemann-invariant conditions normal to these boundaries. On the solid boundaries, the no-slip and no-penetration conditions are enforced; $u_{1}=u_{2}=u_{3}$ $=0$, and the normal pressure gradient is set equal to zero. For the temperature, the adiabatic boundary condition is enforced at the solid boundaries.

The initial conditions correspond to the freestream conditions with $u_{1}=u_{2}=u_{3}=0$ on the solid boundaries.
The freestream conditions are given by

$$
\begin{aligned}
& \rho_{\propto}=a_{\infty}=T_{\propto}=1, \\
& u_{1 \propto}=M_{\infty} \cos \alpha, \\
& u_{2 \propto}=M_{\infty} \sin \alpha, \\
& u_{3 \propto}=0, \\
& P_{\infty}=1 / \gamma, e_{\propto}=\frac{1}{\gamma(\gamma-1)}+\frac{M_{\propto}^{2}}{2}
\end{aligned}
$$

where $\alpha$ is the angle of artack.

\section{COMPUTATIONAL SCHEME}

The implicit, upwind, flux-difference splitting, finitevolume scheme is used to solve the unsteady, compressible, thin-layer and Full Reynolds-averaged. NavierStokes equations. The scheme uses the flux-difference splitting scheme of Roe which is based on the solution of the approximate Riemann problem. In the Roe scheme, the inviscid flux difference at the interface of computational cells is split into two parts; left and right flux differences. The spliting is accomplished according to the signs of the eigenvalues of the Roe averaged-Jacobian matrix of the inviscid tluxes at the cell interface. The smooth flux limiter is used to eliminate oscillations at locations of large flow gradients. The viscous-and heaflux terms are linearized and the cross-derivative terms are eliminated in the implicit operator. The viscous terms are differenced using a second-order accurate central differencing. The resulting difference equation is approximately factored and is solved in three sweeps in the $\xi^{1}, \xi^{2}$, and $\xi^{3}$ directions. The computational scheme is coded in the computer program "FTNS-3D"31.

\section{COMPUTATIONAL RESULTS AND DISCUSSTONS}

We consider a rectangular wing of aspect ratio, AR $=5.9$ and a NACA 0012 chordal section, at an angle of attack of $\alpha=4.64^{\circ}$. The flow Reynolds number, $R_{e}$ is $3.2 \times 10^{6}$ (based on the root-chord length) and the Mach number, $M_{\infty}$ is taken as 0.3 for low speed flows. The computational domain consists of a hemispherical boundary which is followed by a cylindrical boundary. The hemisphere center is located at the intersection of the wing root-chord and its trailing edge. Its radius is 15 chords and the cylindrical boundary extends 14 chords in the downstream direction beyond the trailing edge. A C$O$ grid of $231 \times 65 \times 65$ grid points in the streamwise wraparound, spanwise and normal directions, respectively, is generated by using transfinite interpolation. The minimum grid specings normal to the wing surface at the leading-edge and tip regions are chosen to be $10^{-5}$. The grid points close to the trailing edge are clustered up to 5 chords in the downstream direction. The reason is that for the present work our interest is focused on the resolution of the near-vortex-wake fow. Figure 2 shows a typical grid for the wing and its near wake. The flow is considered to be laminar until the chord station of 0.05 from the wing leading edge. Thereafter, the turbulent model is 
turned on to mimic the transition to turbulent flow. This location of the transitional flow has been experimentally determined by Yip and Shubert ${ }^{32}$.

\section{Thin-Layer and Full Navier-Stokes Solutions}

The flow around the rectangular wing is solved using the thin-layer NS equations and the full NS equations. The computations are carried out three times using the same grid described above and the implicit upwind scheme. In the first and second times, the thin-layer NS equations are solved using the implicit upwind scheme with and without the smooth flux limiter. In the third time, the full NS equations are solved using the implicit upwind scheme without the smooth flux limiter. Next, we compare the results of these three computions with each other and with the experimental data of Ref. 32.

Figure 3 shows a comparison of the history of the logarithmic residual error and the lift coefficient of these computations. The thin-layer computation with a flux limiter (TL-limiter) shows a drop of the residual error of about five orders of magnitude after 8000 iteration steps. The thin-layer computation without a flux limiter (TL-no limiter) shows a drop of the residual error of the same order of magnitude as that of the TL-limiter computation after 10,800 iteration steps. The full Navier-Stokes computation without a flux limiter (FNS-no limiter) shows a drop of the same order of magnitude as that of the TL-limiter computation after 8,900 iteration steps. The lift coefficient of the three computations is 0.34090 (TLlimiter), 0.35354 (TL-no limiter) and 0.35250 (FNS-no limiter). In the next table, we show comparison of the three computations and the experimental data for the normal force coefficient, $C_{N}$, the pitching-moment coefficient about the leading edge, $C_{M}$, and the drag coefficient, $C_{D}$. The results of the case of next section for the tip-jet control are also included in the table. The results show that

\begin{tabular}{ll|l|l} 
& \multicolumn{1}{c|}{$\mathrm{C}_{\mathrm{N}}$} & \multicolumn{1}{c|}{$\mathrm{C}_{\mathrm{M}}$} & $\mathrm{C}_{\mathrm{D}}$ \\
\cline { 2 - 4 } TL-limiter & 0.34128 & -0.07581 & 0.01857 \\
TL-no limiter & 0.35374 & -0.08161 & 0.01682 \\
FNS-no limiter & 0.35272 & -0.08137 & 0.01702 \\
Experiment & 0.35 & -0.0825 & $\mathrm{~N} / \mathrm{A}$ \\
FNS-no limiter & 0.3682 & -0.08667 & 0.01732 \\
(tip jet control) & & &
\end{tabular}

the $\mathrm{C}_{N}$ of the FNS-no limiter computation is the closest to the experimental $C_{N}$ with an error of $+0.78 \%$. The corresponding error for the TL-no limiter computation is $+1.07 \%$ and for the TL-limiter computation is $-2.49 \%$. The error in the computed $C_{M}$ in comparison with the experimental value is $-1.37 \%$ for the NS-no limiter computation, $-1.08 \%$ for the TL-no limiter computation and $-8.11 \%$ for the TL-limiter computation.

Figure 4 shows a comparison of the computed surfacepressure-coefficient distribution in the chordwise direction at different spanwise stations with the same experimental data. The present computed results are in good agreement with those of the experimental data with the exception of the peak suction pressure at the wing leading edge. This is attributed to the simple modeling of the transitional flow at this location, wherein a simple algebraic turbulent model is tumed on. At the spanwise station of 0.9883 in the tip region, it is noticed that the computed $C_{p}$ using the TL-no limiter and FNS-no limiter computations are in excellent agreement with the experimental data than that of the TL-limiter computation, particulary in the chordwise direction as of $\mathrm{X} / \mathrm{C}=0.3$.

Figure 5 shows a comparison of the spanwise variation of the total pressure-loss contours at chord-stations range of $\mathrm{X} / \mathrm{C}=0.9-5.0$ covering the evolution of vortex wake. The range of the total pressure-loss contours shows that the tip vortex reaches its maximum strength very close to the downstream side of the trailing edge. Thereafter, the tip-vortex core expands due to the viscous diffusion and moves inboards while growing in size. It is also observed that the wake thickness increases and moves upwards. The TL-no limiter results of the vortex core and wake shear layer show less viscous diffusion and more inboard motion than those of the TL-limiter results. The FNS-no limiter results show a little better resolution of the vortex core and wake shear layer than that of the $\pi L$. no limiter results. However, the inboard motion of the vortex core and wake shear layer is the same as that of the TL-no limiter results. Hence, it is concluded that the flux limiter is responsible for the vortex-core and wake shear-layer diffusions and their small inboard motions. In Fig. 6, the particle traces for the wing tip flow and the vortex wake flow are shown for the TL-limiter and FNSno limiter computations. Again, the TL-limiter results show the larger diffusion of the tip vortex than that of the FNS-no limiter results.

\section{Active Injection Control of Tp Vortex}

The hazardous effects of the tip vortex on trailing aircraft is very significant as it has been demonstrated in Ref. 27. Several ideas exist for passive and active control of the tip vortex in order to alleviate its hazardous effects. The challenging issue here is to diffuse the tip vortex without decreasing the lifting force of the leading aircraft. Our first attempt to accomplish these objectives is to use active injection from a slot along the wing tip. The slot is located on the wing-tip surface and extends from the chord station $X / C=0.2$ to the chord station $X / C=0.6$. The direction of the jet blowing is downstream at $45^{\circ}$ which is measured from the $x$-axis. A constant mass-flow-rate coefficient of 0.015 is used for the jet blowing through the wing-tip slot The solution of this case is obtained by using the FNS-no limiter computation. Two types of initial conditions have been used. In the first, the control starts from the uniform flow conditions while in the second, the control starts from the FNS-no limiter solution of the preceding section. The final steady flow

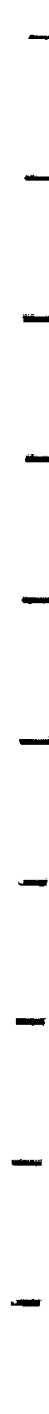

$\rightarrow$

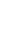

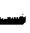

is 
solution for both cases showed the same results. Here, only the results of the first case are shown.

Figure 7 shows the history of the residual error and lift coefficient for 16,000 iteration steps. The spikes shown in the residual error graph are due to the step increases of the mass-flow rate from 0.005 to 0.01 and finally to 0.015 . The residual error drops three orders of magnitude and the lift coefficient shows a value of 0.3680 . The lift coefficient is $4.4 \%$ higher than that of the FNS-no limiter computation without injection. The values of $C_{N}, C_{M}$ and $C_{D}$ are included in the table of the preceding section. The $C_{D}$ coefficient shows $1.76 \%$ increase than that of the FNS-no limiter computation without injection. In Fig. 8, the pressure coefficient is shown at the spanwise stations near the wing tip for comparison with the experimental data of the case without injection. It is observed that only the tip-section $C_{p}$ near the trailing edge shows a slight decrease from that of the experimental data.

Figure 9 shows the spanwise variation of the total pressure-loss contours at chord stations from $X / C=0.95$ to $\mathrm{X} / \mathrm{C}=5.0$. A comparison of these results with those of Fig. 5 reveals the excellent effect of the tip-injection control in dissipating the tip vortex. This is also seen in Fig. 10 of the particle traces in comparison with those of Fig. 6.

Figure 11 shows a blow-up of the total pressureloss contours at the wing tip covering chord stations from $X / C=0.2$ to $X / C=0.9$. It is observed that the tip vortex existing at $\mathrm{X} / \mathrm{C}=0.2$ (at the starting location of the slot) is quickly diffused by the blown jet. However, by the end of the slot length at $X / C=0.6$, the tip vortex is recovering but with small strength. It is concluded that tip-injection control is effective in diffusing the tip vortex and moreover it is very important to study the effect of the injection-slot length in the future. Other parameters need to be included in the future study such as the direction of injection, the mass-flow rate and the width of injection.

\section{CONCLUDING REMARKS}

The thin-layer and full NS equtions have been used to accurately solve for the near-vortex-wake flow around a large-aspect-ratio rectangular wing. The flow has been computed by using the thin-layer equations with and without flux limiters and the NS equations without flux limiters, all on the same grid. The results show the substantial effects of the flux limiter in diffusing the tip vortex and the trailing-edge shear layer and in their small inboard motions. The full Navier-Stokes computation without flux limiters show the best results in comparison with the experimental data. Also, it has been demonstrated that tip-injection control is substantially effective in diffusing the tip vortex without reduction of the wing lift coefficient Although the drag coefficient slightly increased the lift to drag ratio increased. Currently, work is underway to conduct an extensive parametric sudy on the tipinjection control and to study its effect on a trailing wing. Passive-control methods are also being investigated.

\section{ACKNOWLEDGEMENT}

For the first two authors, this research work has been supported by the NASA Langley Research Center under Grant No. NAG-1-994. The computational resources provided by the NASA Langley Research Center and the National Aerodynamics Simulation (NAS) Center also are appreciated.

\section{REFERENCES}

1. Hallock, J. N. and Eberle, W. R., "Aircraft Wake Vortices: A State-of-the-Art Review of the United States R\&D Program," FAA Rept. FAA-RD-77-23, February 1977.

2. Dee, F. W. and Nicholas, O. P.. "Flight Measurements of Wing tip Vortex Motion Near the Ground," CP 1065. British Aeronautical Research Council, London, 1968.

3. Harvey, J. K. and Perry, F. J., "Flowfield Produced by Trailing Vortices in the Vicinity of the Ground," AIAA Journal, Vol. 9, Aug. 1977, pp. 1659-1660.

4. El-Ramly, Z., "Induced Rolling Moment on Trailing Wings," AIAA 10th Fluid \& Plasma-dynamics Conference, Albuquerque, NM. June 1977.

5. Wood, W. D. and McWilliams, T. G., "Wake Turbulence Detection and Economic Impact of Proposed Improvements," SAE Air Transportation Meeting, Washington, D.C., May 1977.

6. Gardoz, L. J., "Federal Aviation Administration FullScale Aircraft Vontex Wake Turbulence Flight Test Investigations: Past, Present, Future," AlAA Paper No. 71-97, January 1971.

7. Cliffone, D. L. and Lonzo, C., Jr., "Flow Visualization of Vortex Interactions in Multiple vortex Wakes Behind Aircraft," NASA TMX 62, 459, June 1975.

8. Olwi, I. A. and Ghazi, M. A., "An Experimental Investigation of the Turbulence Effect of a Leading Wing on a Trailing Aircraft" " ALAA 91-3309-CP, AIAA 10th Applied Aerodynamics Conference, September 1991, pp. 830-837.

9. Liu, H.-T., "Effects of Ambient Turbulence on the Lifespan of a Trailing Vortex Wake," Quest Integrated, Inc., Technical paper No. 268, Kent, Washington, October 1991.

10. Liu, H.-T., Hwang, P.A. and Smsty, R.A., "Physical Modeling of Ground Effects on Vortex Wakes," Quest Integrated, Inc., Technical paper No. 274, Kent, Washington, October 1991.

11. Chorin, A. J. and Bernard, P. S., "Discretization of a Vortex Sheet, with an Example of Roll-up," Journal of Computational Physics, Vol. 13, November 1973, pp. $423-429$. 
12. Hackett, J. E. and Evans, M. R., "Vortex Wakes Behind High Lift Wings," Journal of Aircraft, Vol. 8, May 1971, pp. 334-340.

13. Yates, J. E., "Calculation of Initial Vortex Roll-Up in Aircraft Wakes," Journal of Aircraft, Vol. 11, July 1974, pp. 397-400.

14. Iversen, J. D. and Bernstein, S., "Trailing Vortex Effects on Following Aircraft," Joumal of Aircraft, Vol. 11, January 1974, pp. 60-61.

15. Rossow, V. J., "Inviscid Modeling of Aircraft Trailing Vortices," Proceedings of NASA Symposium on Wake Vortex Minimization," Washington, D.C., February 1976 , pp. 4-54.

16. Kandil, O. A., Mook, D. T. and Nayfeh, A. H., “Application of the Nonlinear vortex-Lattice Concept to aircraft-Interference Problems," Advances in Engineering Science, NASA CP-2001, Vol. 4, November 1976, pp. 1321-1326.

17. Kandil, O. A., Mook, D. T. and Nayfeh, A. H., "Nonlinear Prediction of the Aerodynamics Loads on Lifting Surface," Journal of Aircraft, Vol. 13, No. 1, January 1976, pp. 22-28.

18. McCormick, B. W., Tangler, J. L. and Sherrieb, H. E., "Structure of Trailing vortices," Journal of Aircraft, Vol. 5, No. 3, May-June 1968, pp. 260-267.

19. Bilanin, A. J., Teske, M. E. and Williamson, G. G., "Vortex Interactions and Decay in Aircraft Wakes," AIAA Journal, Vol. 15, No. 2, Feb. 1977, pp. $250-260$.

20. Bilanin, A. J., Teske, M. E. and Hirsh, J. E., "Neutral Atmospheric Effects on the Dissipation of Aircraft Vortex Wakes," AlAA Journal, Vol. 16, No. 9, September 1978, pp. 956-961.

21. Liu, C. H. and Ting, L., "Interaction of Decaying Trailing vortices in spanwise Shear Flow," Computer and Fluids, Vol. 15, No. 1, 1987, pp. 77-92.
22. Weston, R. P., Ting, L. and Liu, C. H., "Numerical Studies of the Merging Vortices," ALAA 86-0557, January 1986.

23. Liu, C. H., Tavantzis, J. and Ting, L., "flumerical Studies of Motion and Decay of vortex Filaments," AIAA Journal, Vol. 24, No. 8, August 1986, pp. 1290-1297.

24. Liu, C. H., Krause, E. and Ting. L., "VortexDominated Flow with Viscous Core Structure," AIAA 85-1556, invited paper, July 1985.

25. Greene, G. C., "An Approximate Model of Vortex Decay in the Amosphere," Joumal of Aircraft, Vol. 23, No. 7, July 1986, pp. 566-573.

26. Greene, G. C., Lamar, J. E. and Kubendran, L. R., "Aircraft Vortices: Juncture, Wing and Wake," AIAA 88-3743, July 1988.

27. Kandil, O. A., Wong, T. C. and Liu, C. H., "Analysis and Computations of Trailing Vortices and their Hazardous Effects," WAKE VORTEX SYMPOSIUM, FAA, Washington, D.C., October 1991.

28. Cebeci, T., "Calculation of Compressible Turbulent Boundary Layers with heat and Mass Transfer," AlAA Paper 70-741, June 1970.

29. Baldwin, B. S. and Lomax, H., "Thin-Layer Approximation and AJgebraic Model for Separated Turbulent Flows," AIAA Paper 78-257, January 1978.

30. Wong, T. C. Kandil, O. A. and Liu, C. H., "NavierStokes Computations of Separated Vortical Flows Past Prolate Spheroid at Incidence," AIAA 89-0553, January 1989.

31. Kandil, O. A., Kandil, H. A. and Liu, C. H., "Supersonic Quasi-Axisymmetric Vortex Breakdown," AIAA 91-3311-CP, Sept 1991, Vol. II, pp. $851-863$.

32. Yip, L. P. and Shubert, G. L., "Pressure Distributions on a 1-By 3-Meter Semispan Wing at Sweep Angles from $00^{\circ}-40^{\circ}$ in Subsonic Flow," NASA TN D-8307, December 1976. 
Figure 1. Types of interference of Trailing Vortex Wake; (1) cross-track penetration, (2) along-track penetration between tip vortices, and (3) along-track penetration through tip vortex center.

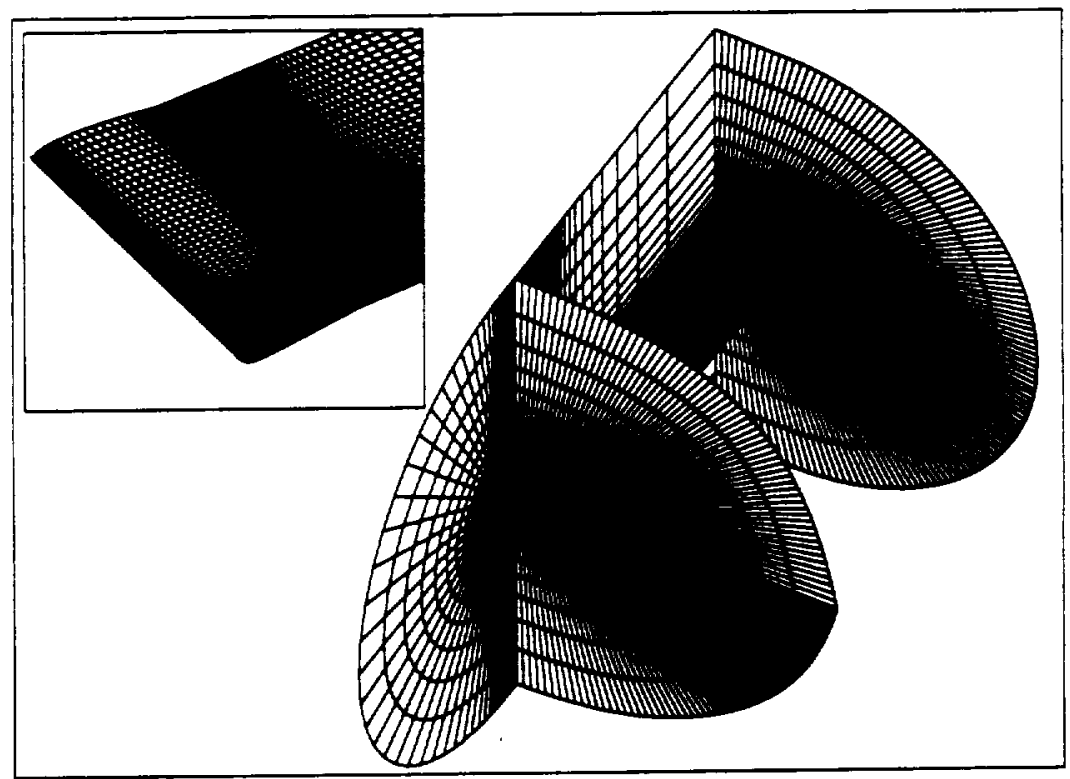

Figure 2. Typical computational grid for the wing 

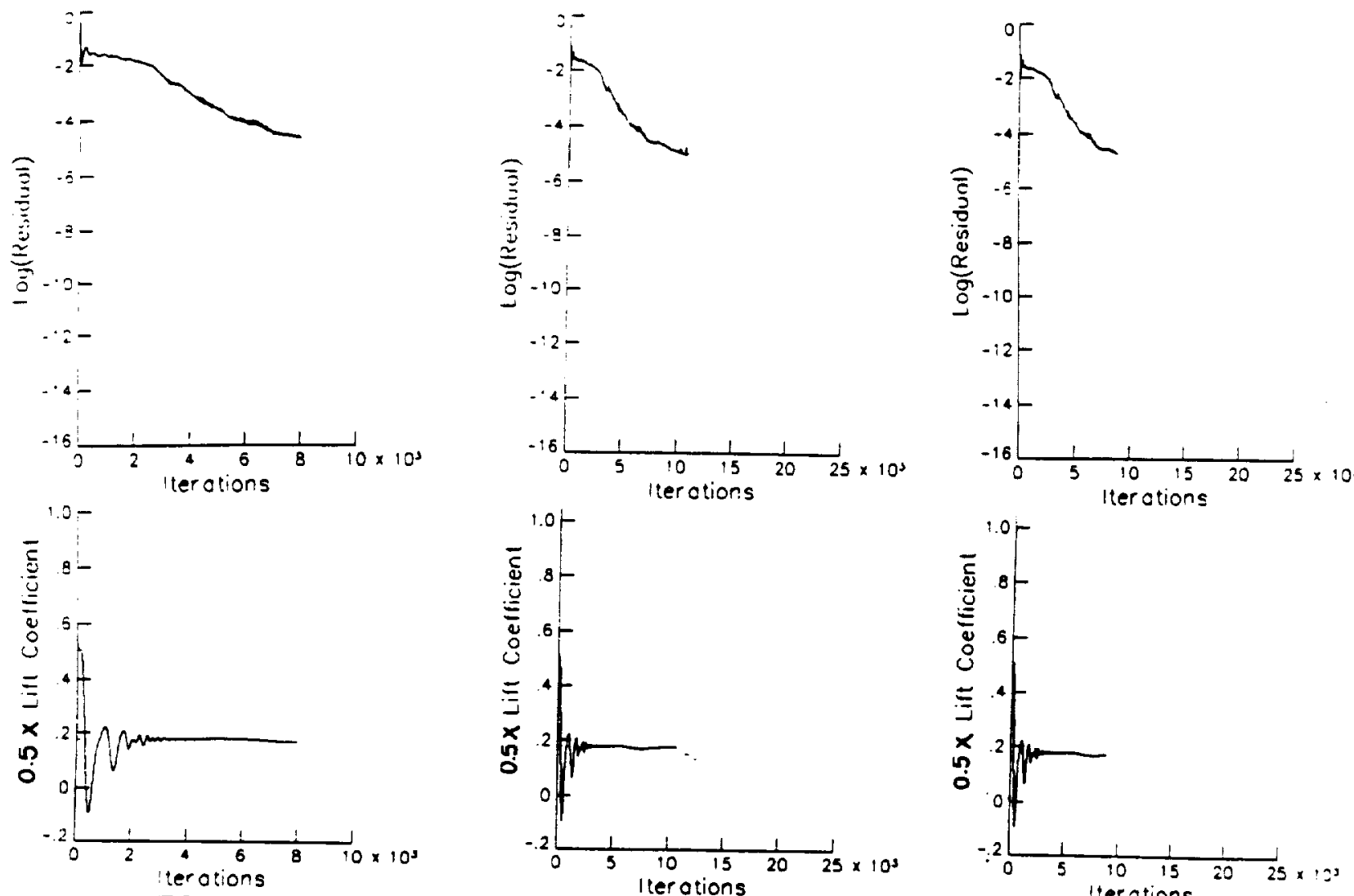

T.L.-limiter

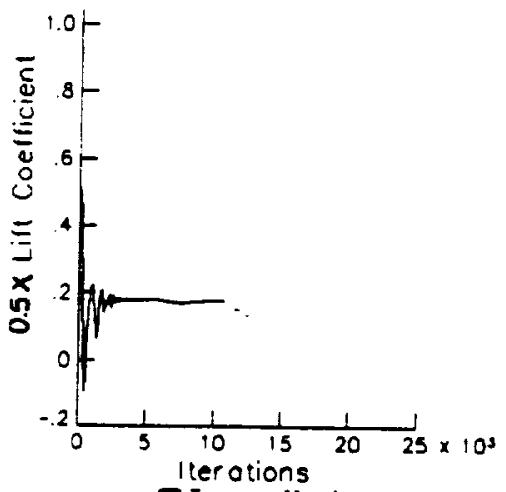

T.L.-no limiter

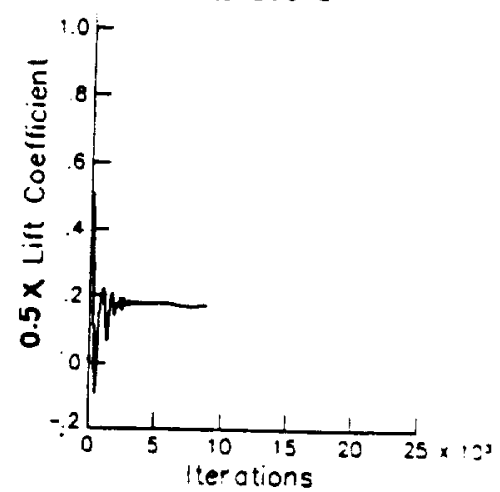

F.N.S.-no limiter

Figure 3. Iteration history of residual error and lift coefficient.
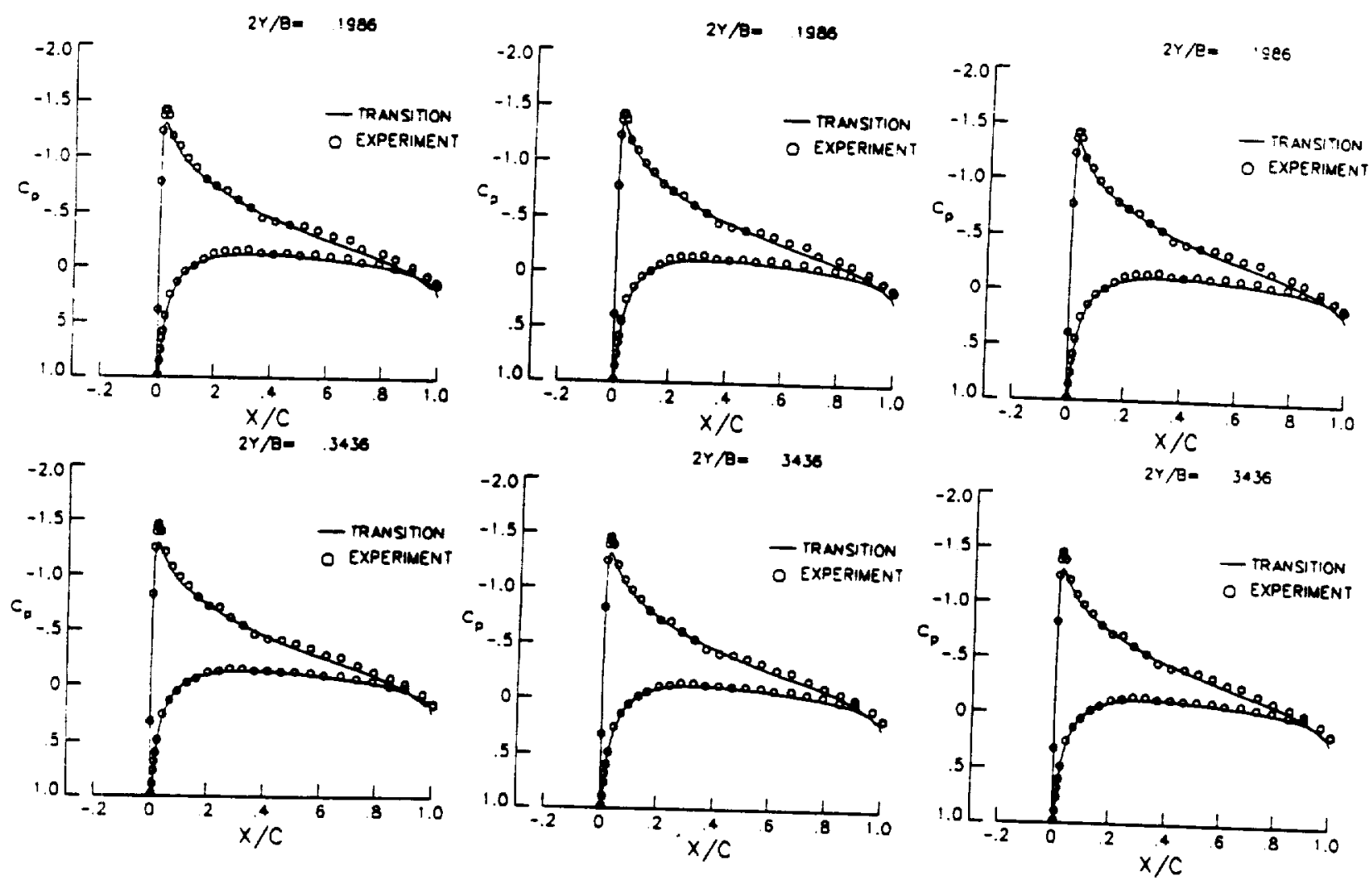

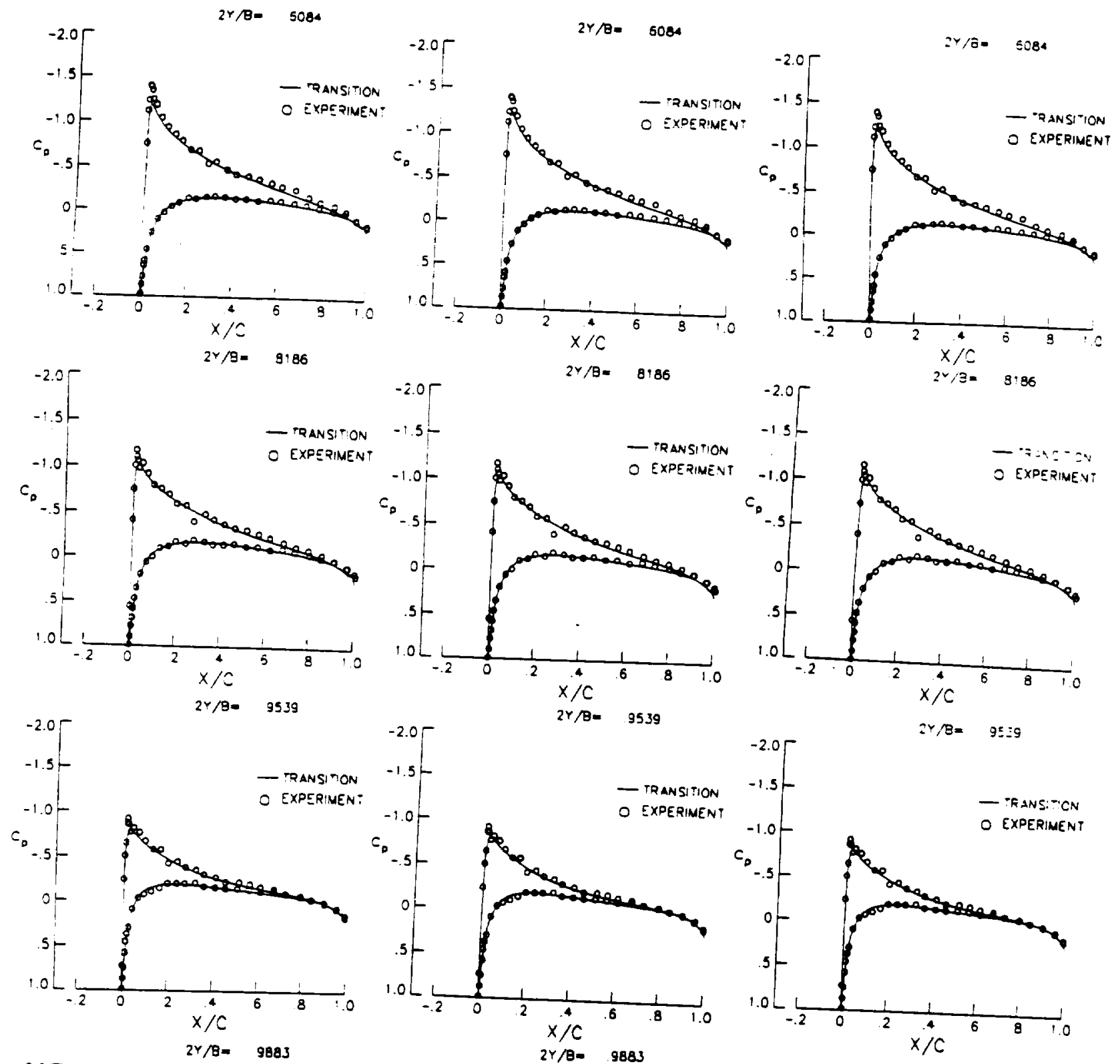

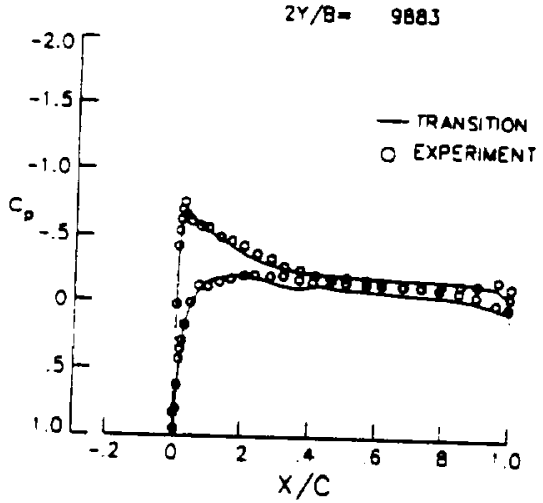

T.L.-limiter

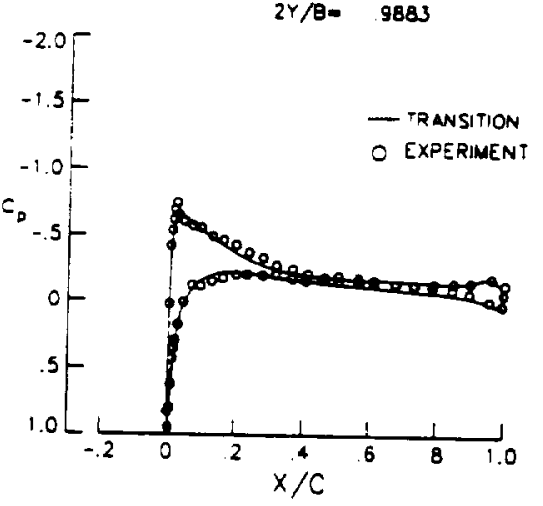

T.L.-no limiter

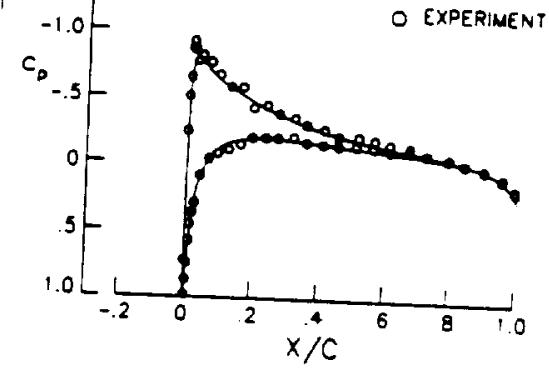

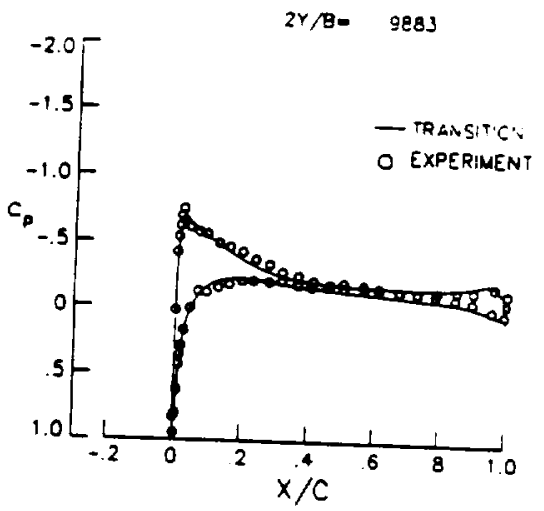

F.N.S.-no limiter

Figure 4. Chordwise surface-pressure distribution at spanwise stations for an isolated wing and comparison with experimental data. 

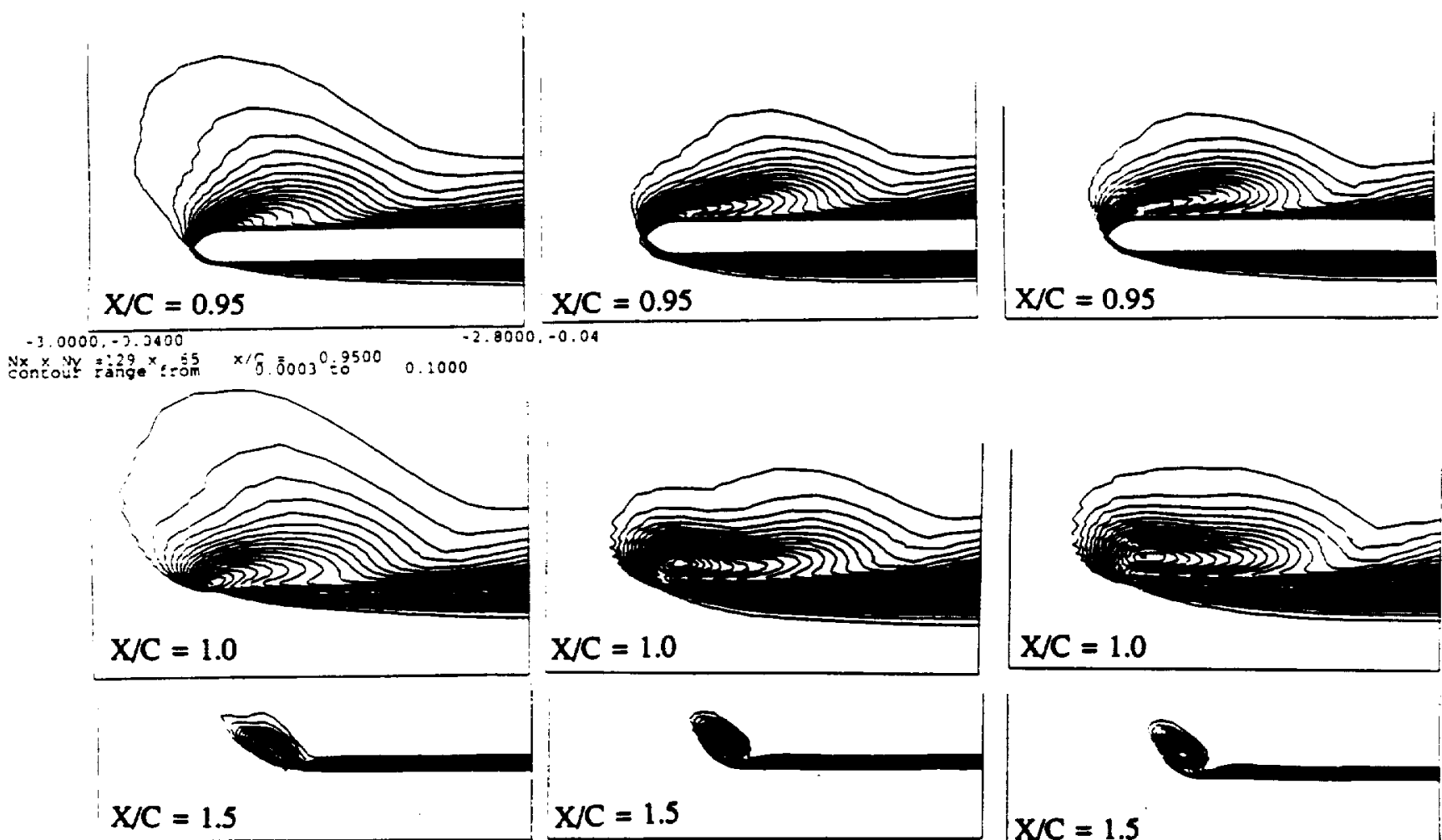

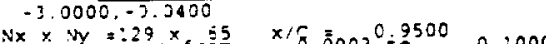

$-3.5000,-0.2500$

$\mathrm{X} / \mathrm{C}=1.5$

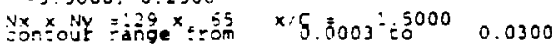

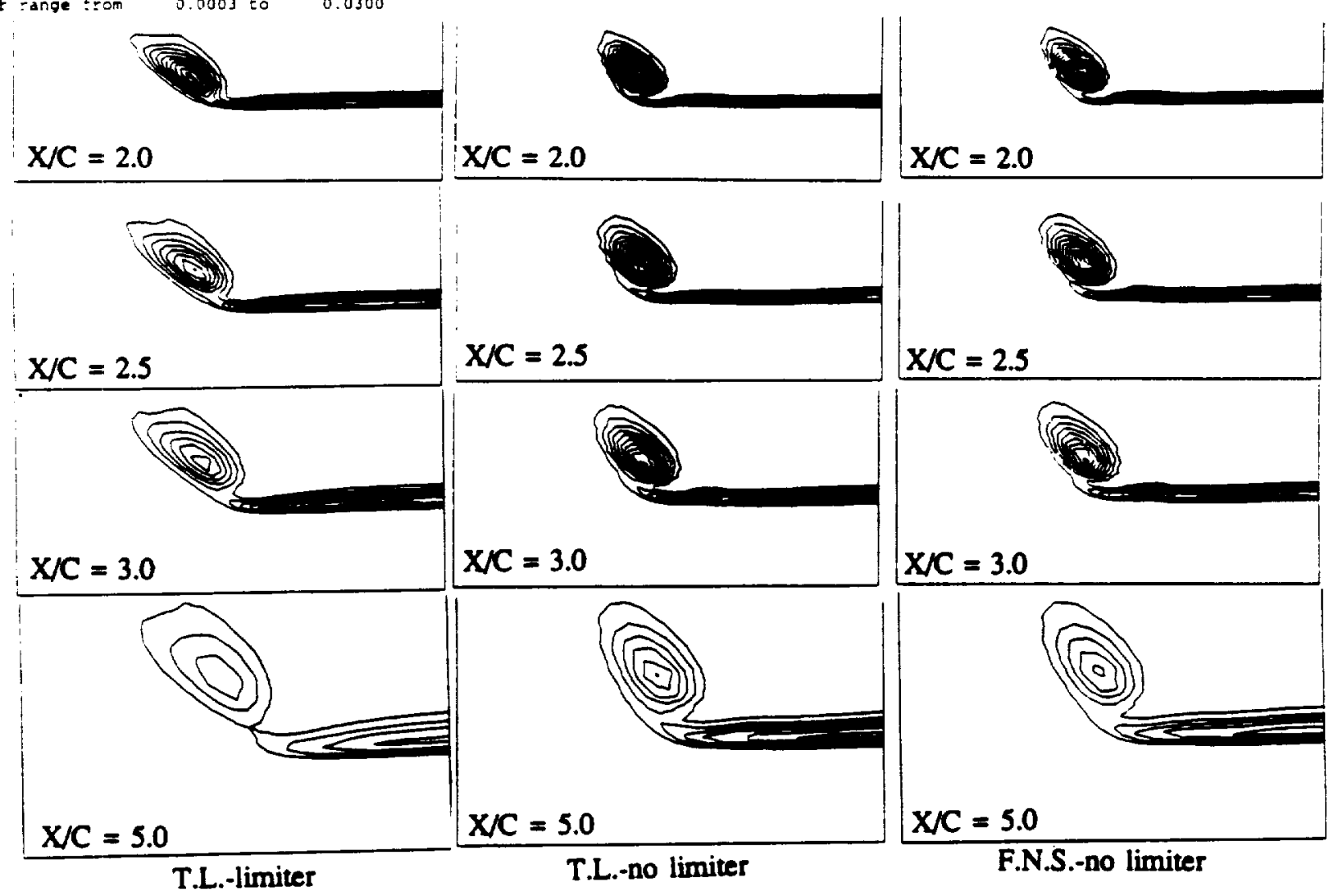

T.L.-limiter

T.L.-no limiter

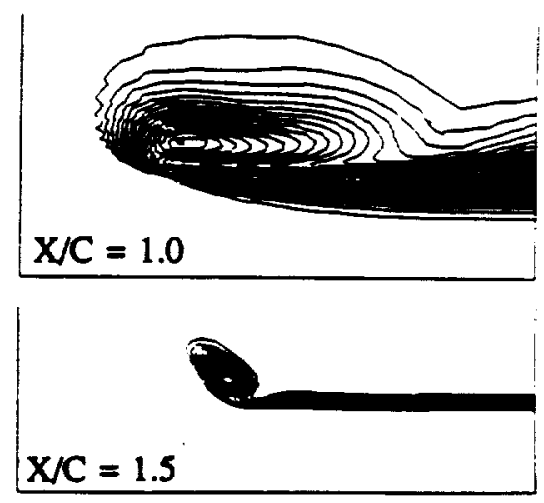

Figure 5. Spanwise variation of total pressure-loss contours at chordwise stations on the wing and its vortex wake. 

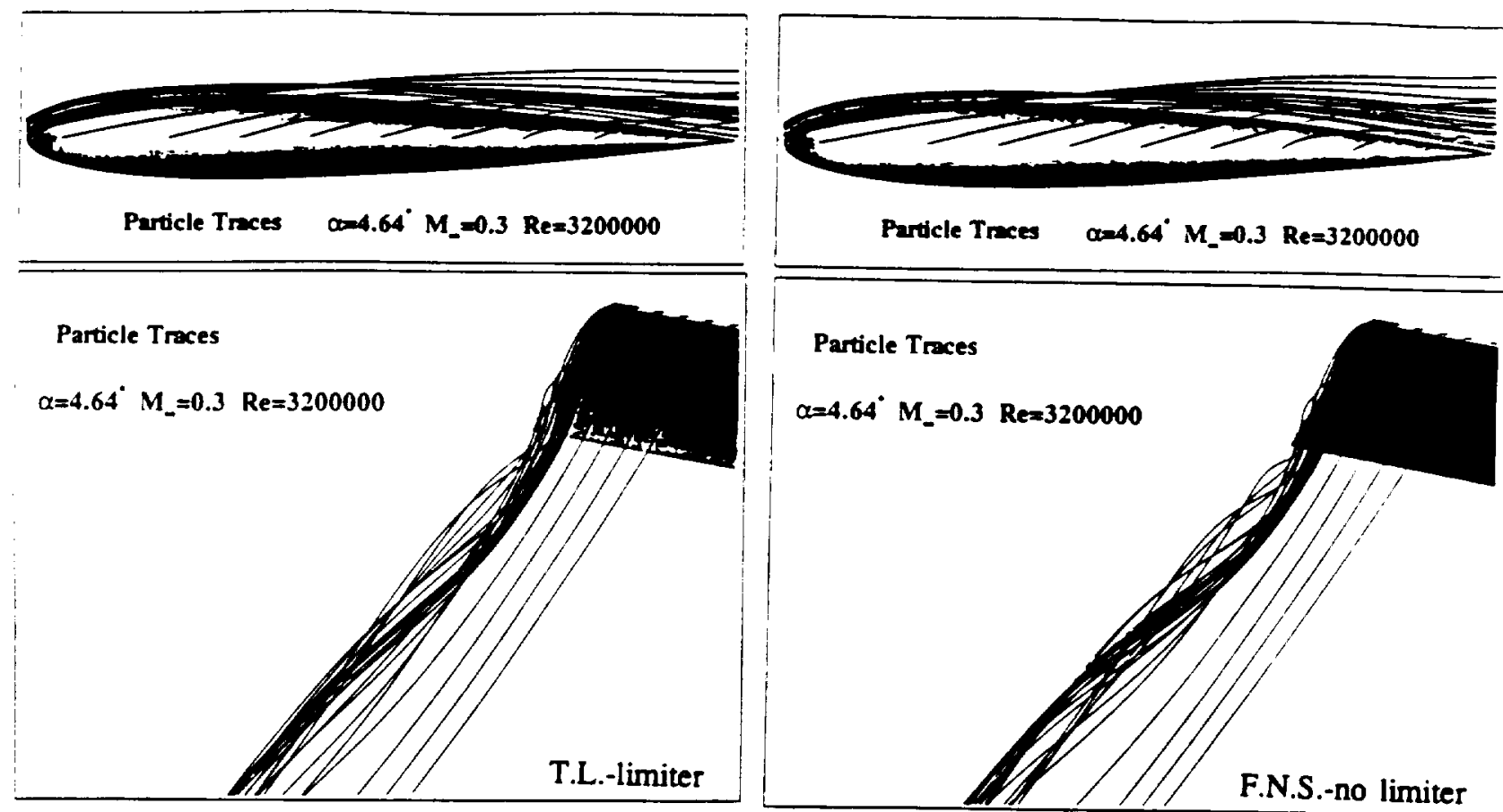

Figure 6. Particle traces for the wing tip and its vortex wake.
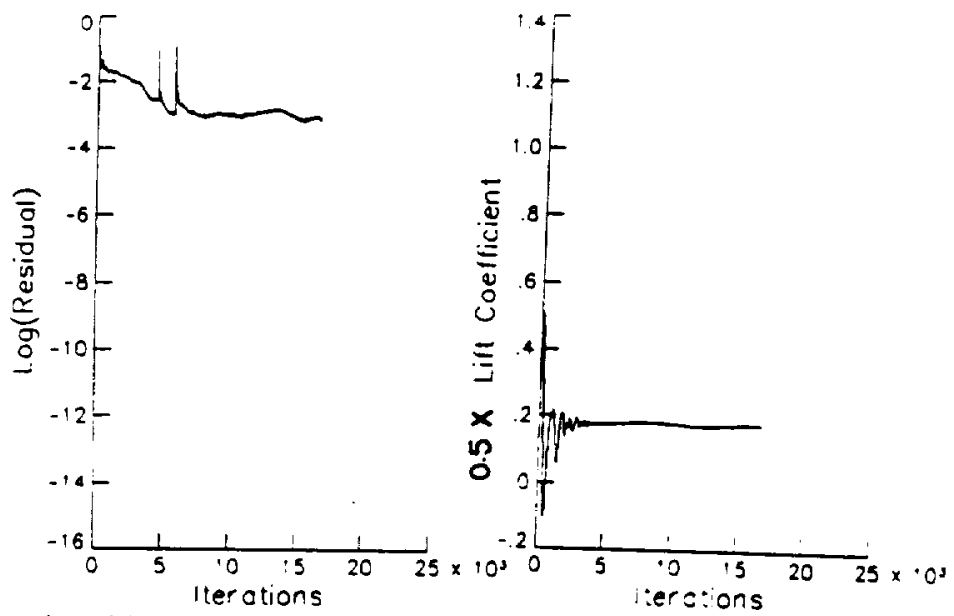

Figure 7. Iteration history of residual error and lift coefficient for the wing with tipinjection control (F.N.S. no limiter).
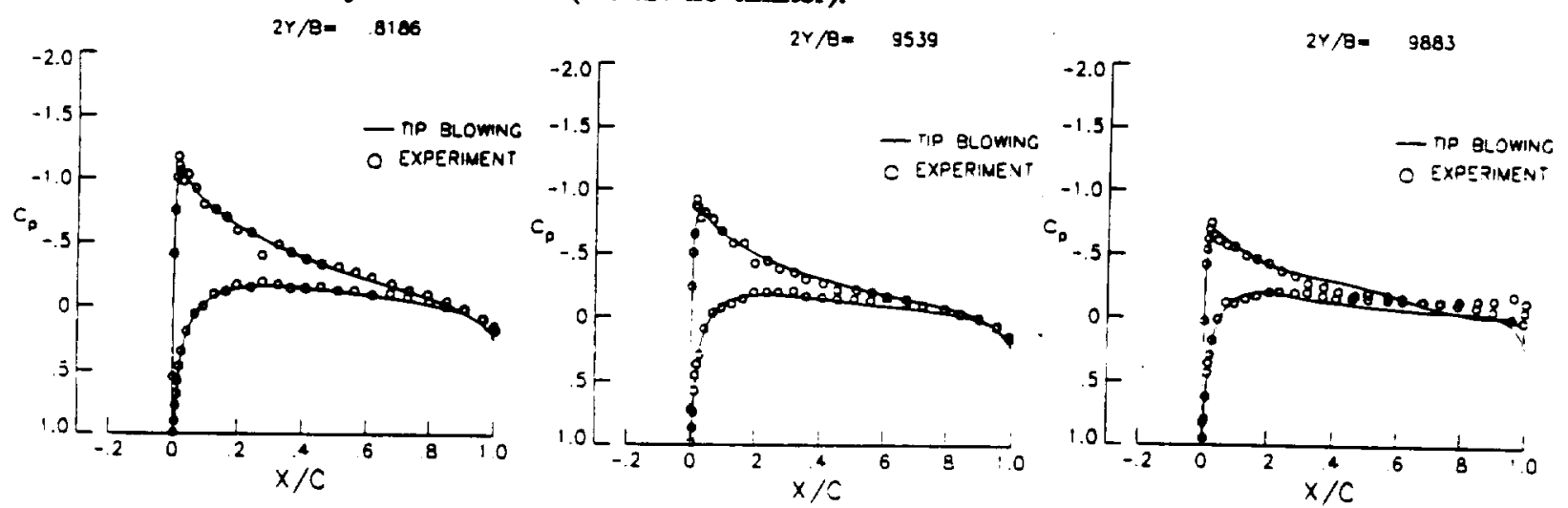

Figure 8. Chordwise surface-pressure distribution at spanwise stations for an isolated wing with tip-injection control (F.N.S. no limiter). 


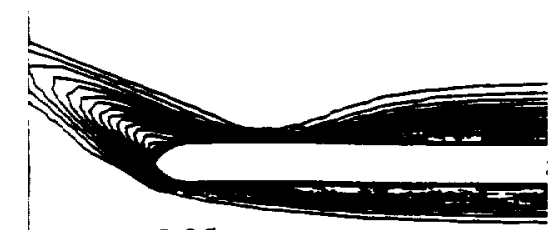

$X / C=0.95$

$-3.0000,-0.0400 \quad-2.350 .-2.2: 2$

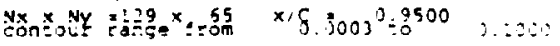

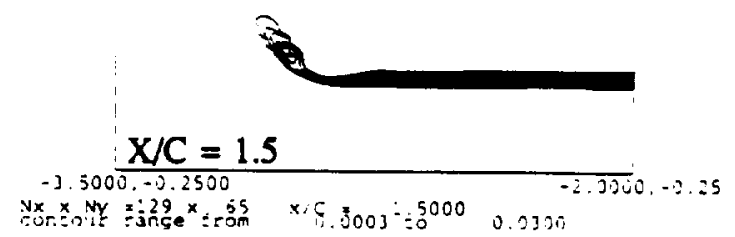

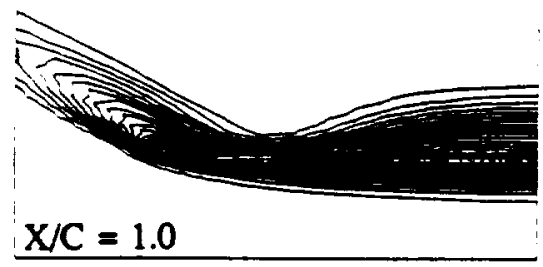

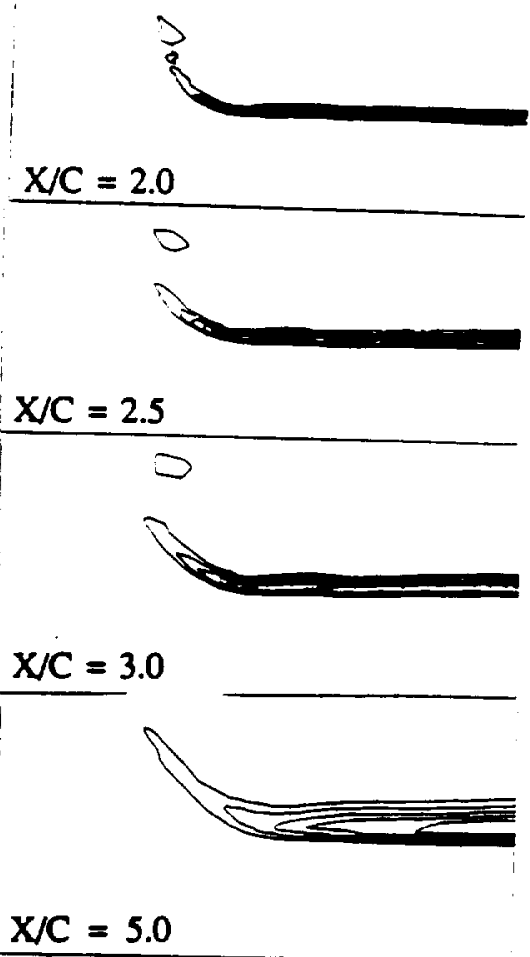

Figure 9. Spanwise variation of total pressure-loss contours at chordwise stations on the wing with tip-injection control and its vortex wake (F.N.S.-no limiter).

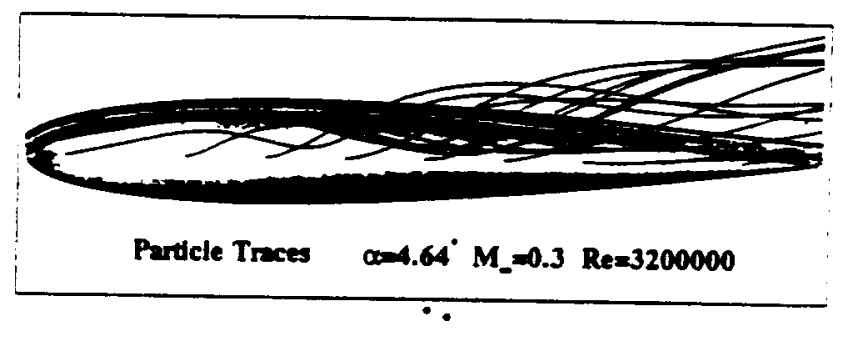

Figure 10. Particle traces for the wing tip with tip-injection control and its vortex wake

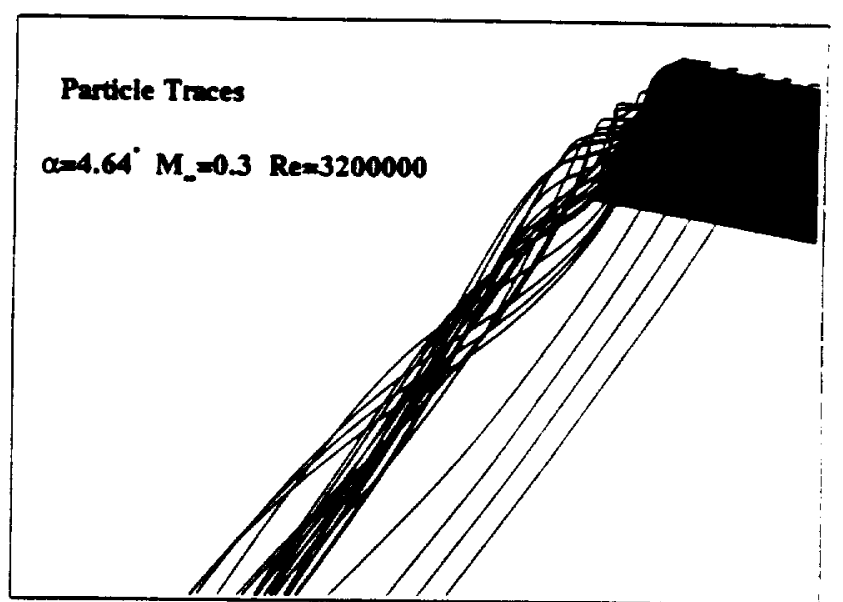

(F.N.S.-no limiter). 


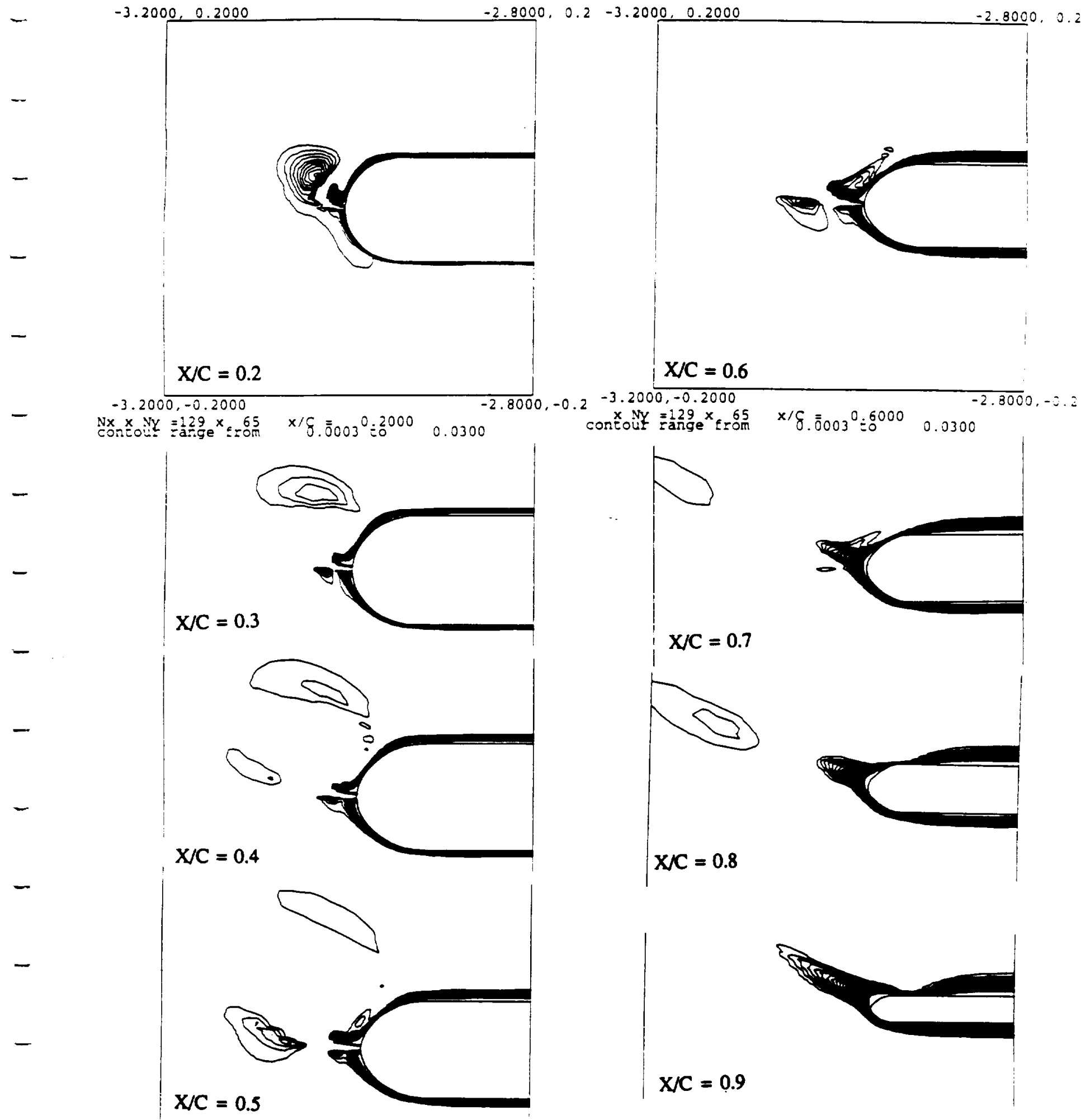

Figure 11. Spanwise variation of total pressure-loss contours at chordwise stations on the wing with tip-injection control (F.N.S.-no limiter). 


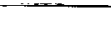


:

AIAA-92-4426-CP

\section{ACTIVE CONTROL OF ASYMMETRIC VORTICAL FLOWS AROUND CONES USING INJECTION AND HEATING}

Osama A. Kandil and Hazem H. Sharaf

Old Dominion University, Norfolk, VA 23529

C. H. Liu

NASA Langley Research Center, Hampton, VA 23665

\section{AIAA Atmospheric Flight Mechanics Conference Hilton Head, SC, August 10-12, 1992}

- For permission to copy or republish, contact the American Institute of Aeronautics and Astronautics 370 L'Entant Promenade, S.W., Washington, D.C. 20024 



\author{
Osame A. Kandil" and Hezem H. Sharaf* \\ Old Dominion University, Norfolk, VA 23529 \\ and \\ C.H. Liu \\ NASA Langley Research Center, Hampton VA 23665
}

\begin{abstract}
The effectiveness of certain active-control methods for asymmetric flows around circular cones is investigated by using computational solution of the unsteady, compressible full Navier-Stokes equations. Two main methods of active control which include flow injection and surface heating are used. For the flow-injection-control method, flow injection is used either in the normal direction to the surface or in the tangential direction to the surface. For the surface-heating-control method, the temperature of the cone surface is increased. The effectiveness of a hybrid method of flow control which combines normal injection with surface heating has also been studied. The Navier-Stokes equations, subjected to various surface boundary conditions, are solved by using an implicit, upwind, flux-difference splitting, finite-volume scheme for locally-conical flow solutions.
\end{abstract}

\section{INTRODUCTION}

The problems of prediction, analysis and control of asymmetric vortical flows around slender pointed bodies are of vital importance to the dynamic stability and controllability of missiles and fighter aircraft. The onset of flow asymmetry occurs when the relative incidence (ratio of angle of attack to nose semi-apex angle) of pointed forebodies exceeds certain critical values. At these critical values of relative incidence, flow asymmetry develops due to natural and/or forced disurbances. The origin of natural disturbances may be a transient side slip, an acoustic disturbance, or similar disturbance of short duration. The origin of forced disturbances is geometric perturbations due to imperfections in the nose geometric symmetry ar similar disturbances of permanent nature. In addition to the relative incidence as one of the influential parameters for the onset of flow asymmetry, the freestream Mach number, Reynolds number and shape of the body-cross sectional area are also important param-
eters.

In several recent papers by Kandil et al. ${ }^{14}$, the unsteady, thin-layer, compressible Navier-Stokes equations have been used to simulate steady and unsteady, asymmetric vortex flows, including their passive control,

\footnotetext{
Professor and Eminent Scholer, Dept. of Mechnnical Engineering and Mechenica, Aseociste Fellow AlAA.

-.. Gradunte Rescarch Assistox, Member AMA.

-.. Group Leader, Theoretical Flow Physica Branch, Aleocime Fellow AIAA.

Copyright 1992 by Osama Kandil, Publishod by The American Instiute of Aemonaties and Astronautics, Inc., with permission.
}

around cones with different cross-sectional shapes. The emphasis of these papers was extensive computational studies of the parameters which influence the asymmetric flow phenomenon and its passive control. Since the computational cost associated with the solution of threedimensional-flow problems at reasonable flow resolution is very expensive, all the computational solutions were obtained using a locally-conical flow assumption. Such an assumption reduces the problem solution to that on two conical planes, which are in close proximity of each other, and hence it reduces the computational cost by an order of magniude. Moreover, such solutions still provide extensive understanding of the flow physics since one can use very fine grids for reasonable flow resolution. These studies showed that asymmetric flow solutions were unique irrespective of the type of flow disturbance; a random disturbance in the form of a machine round-off error or a controlled disturbance in the form of a short-duration side-slip disturbance. Unsteady asymmetric flow solutions with perfectly periodic vortex shedding were successfully simulated, and the solutions were unique irrespective of the computational scheme used. It has also been shown that as the Mach number was increased, the flow asymmetry was decreased, and as the Reynolds number was increased the flow asymmetry was increased. Moreover, the cross-sectional shape of the cone has been shown to be a very influential parameter on the flow asymmetry. Circular sections produced very strong flow asymmetry and diamond sections produced relatively-weaker flow asymmetry.

In a later paper, by Kandil et a.. ${ }^{5}$, the full NavierStokes solutions were compared with the thin-layer Navier-Stokes solutions. It was shown that the full Navier-Stokes solutions produced thicker free-shear layers and more vortex-core resolution as compared with those of the thin-layer Navier-Stokes equations. In Ref. 5, a few tentative three-dimensional flow solutions were also presented.

Substantial research efforts have recently been devoted for eliminating or alleviating flow asymmetry and its corresponding side force. In the experimental area, several passive-control methods ${ }^{6-3}$ and activecontrol methods ${ }^{9-13}$ have been investigated. Computational simulations have also been used to investigate the effectiveness of several passive-control methods ${ }^{1.5}$ and active-control methods ${ }^{12}$ 14, 15. Various methods of passive control were demonstrated in the above references which include the use of vertical fins along the leeward plane of geometric symmetry, thin and thick side strakes 
with different orientations, and rotamable forebody tips which have variable cross section (from a circular shape at its base to an elliptic shape at its tip). It was shown by Kandil et al. ${ }^{4}$ that side-strakes control is more prectical than the vertical-fin control since the former was more effective over a wide range of angle of attack than the latter. Moreover, side-strake control provided an additional lifting force. However, the effectiveness of the side-strake control terminates at very high angles of attack for the considered strake geometry and flow conditions.

Various active-control methods have been used which include forebody blowing and movable forebody strakes. The forebody blowing methods include forward blowing. normal blowing, aft blowing and tangential blowing. The main concept of forebody blowing is to control fow separation on the forebody and to create yawing forces and moments which can be utilized in controlling the body.

In the present paper, we investigate the effectiveness of two main methods of active control which include flow injection and surface heating. The study of flow-injection control covers normal and tangetial injection. Moreover. a hybrid method of flow control which combines surface heating and normal injection methods is also investigated. At this stage of research, the flow control is aiming at either rendering the vortical asymmetric flow symmetric or rendering the surface-pressure distribution symmetric. Computational solution of the unsteady, compressible, full Navier-Stokes equations is used for the present work with the exception of the tangential injection. For the tangential injection, the thin-layer NS equations are used. The computational applications are focused on circularsection cones, and locally-conical flow assumptions are used to substantially reduce the computational cost.

\section{FORMULATION AND COMPUTATIONAL SCHEME}

\section{Full Navier-Stokes Equations}

The conservative form of the dimensionless, unsteady, compressible, full Navier-Stoikes equations in terms of time-independent, body-conformed coordinates $\xi^{1}, \xi^{2}$ and $\xi^{3}$ is given by

$$
\begin{aligned}
& \frac{\partial \overline{\mathrm{Q}}}{\partial \mathrm{t}}+\frac{\partial \overline{\mathrm{E}}_{\mathrm{m}}}{\partial \xi^{\mathrm{m}}}-\frac{\partial\left(\overline{\mathrm{E}}_{\mathrm{v}}\right)_{\mathrm{s}}}{\partial \xi^{\mathrm{t}}}=0 ; \mathrm{m}=1-3, \mathrm{~s}=1-3 \\
& \xi^{\mathbf{m}}=\xi^{\mathbf{m}}\left(x_{1}, x_{2}, x_{3}\right) \\
& \vec{Q}=\frac{\hat{q}}{J}=\frac{1}{j}\left[\rho, \rho u_{1}, \rho u_{2}, \rho u_{3}, \rho e\right]^{t} \\
& \overline{\mathrm{E}}_{\mathbf{m}} \equiv \text { inviscid flux } \\
& =\frac{1}{J}\left[2 \xi^{m} \hat{E}_{k}\right]^{2} \\
& =\frac{1}{j}\left[\rho \mathrm{U}_{m}, \rho \mathrm{u}_{1} \mathrm{U}_{m}+\partial_{1} \xi^{\mathbf{m}} \mathrm{p}, \rho \mathrm{u}_{2} \mathrm{U}_{\mathrm{m}}\right. \\
& \left.+\partial_{2} \xi^{\mathrm{m}} \mathrm{p}, \rho \mathrm{u}_{3} \mathrm{U}_{\mathrm{m}}+\partial_{3} \xi^{\mathrm{m}} \mathrm{p},(\rho \mathrm{e}+\mathrm{p}) \mathrm{U}_{\mathrm{m}}\right]^{\mathrm{s}}
\end{aligned}
$$

$$
\begin{aligned}
& \left(\bar{E}_{v}\right)_{s} \equiv \text { viscous and heat-conduction } f u x \text { in } \xi^{*} \\
& \text { direction }
\end{aligned}
$$

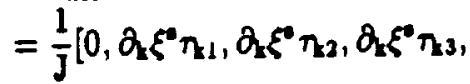

$$
\begin{aligned}
& \left.\partial_{k} \xi^{*}\left(u_{n} n_{k}-q_{k}\right)\right]^{i} ; \quad k=1-3, n=1-3 \\
& \mathrm{U}_{\mathrm{m}}=2 \xi^{\mathrm{m}} \mathrm{u}_{\mathrm{k}}
\end{aligned}
$$

The three momentum elements of Eq. (5) are given by

$$
\begin{aligned}
\partial_{k} \xi^{*} n_{j j} & \equiv \frac{M_{\propto \mu} \mu}{R e}\left[\left(\partial_{k} \xi^{2} \partial_{j} \xi^{n}-\frac{2}{3} \partial_{j} \xi^{*} \partial_{k} \xi^{n}\right) \frac{\partial u_{k}}{\partial \xi^{n}}\right. \\
& \left.+\partial_{k} \xi^{\bullet} \partial_{k} \xi^{ \pm} \frac{\partial u_{j}}{\partial \xi^{\natural}}\right]
\end{aligned}
$$

The last element of Eq. (5) is given by

$\partial_{k} \xi^{\prime}\left(u_{p} \tau_{k p}-q_{k}\right) \equiv \frac{M_{\infty \alpha} \mu}{R e}\left[\left(\partial_{k} \xi^{\bullet} \partial_{p} \xi^{n}-\frac{2}{3} \partial_{\alpha} \xi^{\prime} \partial_{k} \xi^{n}\right) u_{,} \frac{\partial u_{k}}{\partial \xi^{n}}\right.$

$\left.+\partial_{k} \xi^{*} \partial_{k} \xi^{n} u_{p} \frac{\partial u_{p}}{\partial \xi^{n}}+\frac{1}{(\gamma-1) P_{r}} \partial_{k} \xi^{*} \frac{\partial\left(a^{2}\right)}{\partial \xi^{n}}\right] ; p=1-3(8)$

The reference parameters for the dimensionless form of the equations are $L, a_{\infty}, L / a_{\infty}, \rho_{\infty}$ and $\mu_{\infty}$ for the length, velocity, time, density and molecular viscosity, respectively. The Reynolds number is defined as $\mathrm{Re}=$ $P_{\propto} V_{\propto} \mathrm{L} / \mu_{\infty}$, where the characteristic length, $L$, is the body length. The pressure, $p$, is related to the total energy per unit mass and density by the gas equation

$$
p=(\gamma-1) \rho\left[e-\frac{1}{2}\left(u_{1}^{2}+u_{2}^{2}+u_{3}^{2}\right)\right]
$$

The viscosity is calculated from the Sutherland law

$$
\mu=T^{3 / 2}\left(\frac{1+C}{T+C}\right), C=0.4317
$$

and the Prandu number $P_{r}=0.72$. In Eqs. (1)-(10), the indicial notation is used for convenience.

\section{Boundary and Initial Conditions}

The boundary and initial conditions vary according to the problem under consideration. The boundary conditions are explicitly satisfied. In general, they include inflow-outfiow conditions and solid-boundary conditions. For problems of flow asymmetry, where the flow is solved throughout the whole computational domain, periodic boundary conditions are used at the plane of geometric symmetry of the problem.

For the asymmetric flow problems around slender bodies and for supersonic inflow-outflow boundary, the Riemann-invariant boundary conditions are used. They require that the inflow variables be at the freestream conditions, and the conical shock enclosing the body be 
captured as part of the solution. For supersonic outflow boundary, the Riemann-invariant boundary conditions require that all flow variables be extrapolated from the interior cells. On the solid boundary, without injection or heating, the no-slip and no-penetration conditions are enforced. Moreover, the zero normal-pressure gradient and adiabatic boundary conditions are enforced. For the present active control problems, the mass-flow rate is specified at the body surface for the normal injection control and the temperature distribution is specified at the surface for the heating control. For the tangential flow injection the mass flow rate and velocity profile are specified at the lip exit (shown in Fig. 10 of next section).

The initial conditions correspond to the uniform flow conditions with $u_{1}=u_{2}=u_{3}=0$ on the solid boundary. These conditions are used to obtain the asymmetric flow solution. Next, the flow control conditions are enforced and the previously obtained asymmetric solution is used for the initial conditions of the active control problem.

\section{Computational Scheme}

The implicit, upwind, flux-difference splitting, finitevolume scheme is used to solve the unsteady, compressible, full Navier-Stokes equations. The scheme uses the flux-difference splituing scheme of Roe which is based on the solution of the approximate Riemann problem. In the Roe scheme, the inviscid flux difference at the interface of computational cells is split into two parts; left and right flux differences. The splitting is accomplished according to the signs of the eigenvalues of the Roe averagedJacobian matrix of the inviscid fluxes at the cell interface. The smooth flux limiter is used to eliminate oscillations at locations of large flow gradients. The viscous-and heatflux terms are linearized and the cross-derivative terms are eliminated in the implicit operator. The viscous terms are differenced using a second-order accurate central differencing. The resulting difference equation is approximately factored and is solved in three sweeps in the $\xi^{1}$. $\xi^{2}$, and $\xi^{3}$ directions. The computational scheme is coded in the computer program "FTNS3D".

For the locally-conical flow solutions, an axial station of $x_{1}=1.0$ is selected and the components of the flowtield vector are forced to be equal between this axial station and another axial station in close proximity to $x_{1}=1.0$. This ensures that the flow variables are locally independent of the axial direction at $x_{1}=1.0$ (Kandil et al. ${ }^{5}$ ).

\section{COMPUTATIONAL APPLICATIONS AND DISCUSSION}

For all the computational applications shown in this section, a $5^{\circ}$-semiapex angle circular cone at 1.8 Mach number and $10^{5}$ Reynolds number is considered. These flow conditions were considered earlier (Kandil et al. ${ }^{4,5}$ ) for the same cone for asymmetric-flow prediction and passive-control methods. The grid is $241 \times 81 \times 2$ points in the wrap around, normal directions and axial direction, respectively. The grid is generated using a modified
Joukowski transformation with a minimum grid size of $10^{-4}$ in the $\xi^{2}$ direction at the body surface. For the tangential flow injection a muldi-block grid has been used and it is explained later on.

\section{Asymmetric Flow, $\alpha=20^{\circ}$}

Figure 1 shows the locally-conical flow solution without any control around the cone at an angle of attack of $20^{\circ}$. The figure shows the total-pressure-loss (TPL) contours and the surface-pressure (SP) coefficient The surface pressure is presented versus the angle $\theta$, which is measured in the clockwise direction from the leeward plane of geometric symmetry. This stable asymmetric flow solution is obtained after 6,000 iteration steps. The source of flow disturbance at these critical flow conditions is the truncation error. As it has been shown earlier (Kandil et al. ${ }^{1}$ ), this solution is unique irrespective of the type of source of the flow disturbance.

Normal Injection Control, $\theta=-22.5^{\circ} \rightarrow-67.5^{\circ}$, I $=$ constant $=0.03, \alpha=20^{\circ}$

Next, a constant mass-fiow injection of $\mathrm{m}=0.03$ is applied normal to the cone surface. The circumferential range of injection ports extends over $\theta=-22.5^{\circ} \rightarrow-67.5^{\circ}$. The solution of the previous asymmetric flow case is used as initial conditions along with modified surface boundary conditions. Figure 2 shows the TPL contours and the SP coefficient from the solution of the controlled flow. Although the vortical flow is still asymmetric, as it is seen from the TPL contours, the SP curve shows a symmetric distribution resulting in a zero side force. The TPL contours show that a primary vortex of smaller height (in comparison to the case of Fig. 1) is still existing on the right side and it is connected through a free-shear layer with the left side of the body surface. The injection flow on the left side of the body decreases the suction pressure on that side making its distribution equal to that on the right side. Normal Injection Control, $\theta=0^{\circ} \rightarrow-67.5^{\circ}$,
由 $=$ constant $=0.03, \alpha=20^{\circ}$

In this case, the constant mass-flow injection of th $=0.03$ is kept fixed while the circumferential range of injection ports is extended to cover the range of $\theta=0^{\circ}$ $\rightarrow-67.5^{\circ}$. Figure 3 shows the TPL contours and SP coefficient of the solution of the controlled flow. The vortical flow is still asymmetric but the SP curve shows a symmetric distribution, which results in a zero side force. It is also noticed that the primary vortex on the right side moves to a larger height (in comparison with the cases of Figs. 1 and 2) and the left-side free-shear layer also moves to the same height level. The SP coefficient curve shows less negative pressure coefficient on the leeward side in comparison with the case of Fig. 2, which produces smaller lifting force. 
Normal Injection Control, $\theta=-675^{\circ} \rightarrow+675^{\circ}$, Variable Masa-Flow Rate, thex $=0.03, \alpha=20^{\circ}$

In this case, the circumferential range of injection ports is extended to cover a symmetric range of $\theta=-$ $67.5^{\circ} \rightarrow+67.5^{\circ}$. Moreover, the injected mass-flow rate is made proportional to the difference in the surface pressure between corresponding points on the right and left side of the body (within $\theta=-67.5^{\circ} \rightarrow+67.5^{\circ}$ ). The maximum injected mass-flow rate, thmax, which corresponds to the maximum pressure difference is restricted to $\mathrm{th}_{\max }=$ 0.03 . Practically, this control method can be achieved by sensing the pressure difference between the right and left ports and using it as a feedback control in order to inject a mass-flow rate which is proportional to that pressure difference. Figure 4 shows the results of this controlled flow. The history of the residual error versus the number of iterations shows the asymmetric solution up to 6,000 iterations and the evolution of the symmetric controlledflow solution up to 7,200 iterations. The corresponding curve of the history of the side force shows that the side force becomes zero at the end of 7,200 iterations. Snapshots of the evolution of the symmetric controlled flow solution, in terms of the TPL contours, are given at the 6,$200 ; 6,400 ; 6,600 ; 6,800 ; 7,000$ and 7,200 iteration step. The vortical flow is rendered symmetric using the present method and the SP coefficient curve shows a symmetric distribution.

Surface Heating Control, $\theta=0 \rightarrow-180^{\circ}$, $T_{1}=7 T_{\infty}, \alpha=20^{\circ}$

For the heating control, the left surface temperature of the cone, in the circumferential range of $\theta=0^{\circ} \rightarrow$ $-180^{\circ}$, has been changed from the adiabatic condition to a constant temperature condition of surface temperabure, $T_{a}=7 T_{\infty}$, where $T_{\infty}$ is the freestream temperamure. Starting from the stable asymmetric flow solution at the 12,000 iteration step, the left surface temperature is raised to $T_{a}=5 T_{\infty}$ and then it is raised further to $T_{1}=7 T_{\infty}$. Figure 5 shows the results of this case which include the history of the residual error and the corresponding history of the side force along with the TPL contours after 24,000 iterations. The side-force curve shows that the side force decreases as the temperature increases until it vanishes for $T_{1}=7 T_{\alpha}$. Although the side force is zero, the vortical flow is still asymmetric. This case shows the effect of increasing the gas viscosity with heating in order to equalize the surface pressure distribution on the right and left sides of the body. This is the first time, we know of, that such an active control method has been applied to control asymmetric flows.
Injection Flow Controh, Varlable Man-Flow

Rate, $\alpha=20^{\circ}-30^{\circ}$

In this case, the effectiveness of the normal injection control of the case of Fig. $4\left(\mathrm{th}=0.03, \alpha=20^{\circ}, \theta=\right.$ $-67.5^{\circ} \rightarrow 67.5^{\circ}$ ) is studied for higher angles of ausck. Figure 6 shows the results of this study which includes the history of the residual error versus the number of iterations, the corresponding history of the side force and the TPL contours along with the SP-coefficient curves for $\alpha=22^{\circ}, 24^{\circ}, 26^{\circ}, 28^{\circ}$ and $30^{\circ}$. The residual error and the side-force curves show the responses for the following history: First, no control is applied until the 12,000 iteration step; second, injection is applied at $\alpha=20^{\circ}$ for 2,000 iterations; third, the angle of attack is increased $2^{\circ}$ each 500 iterations until $\alpha=28^{\circ}$; fourth, at $\alpha=30^{\circ}$ injection is continued for 2,000 iterations. The side-force curve shows zero value in the angle of attack range of $\alpha=$ $20^{\circ}-28^{\circ}$ (corresponding to the iterations range of 14,000 $-16,000)$. When the angle of attack reaches $30^{\circ}$, the normal injection control becomes incapable of achieving flow symmetry. It is interesting to study the snapshots of the TPL contours at these angles of attack. It is noticed that the primary vortices increase in size in the normal direction and their inner boundaries approach each other. At $\alpha=28^{\circ}$, the inner boundaries of the primary vortices wouch each other, and thereafter at $\alpha=30^{\circ}$ the primary vortices become asymmetric. The asymmetric response of the primary vortices at $\alpha=30^{\circ}$ is believed to be due to a strong instability arising due to the strong interaction of the inner shear layers of the primary vortices.

At $\alpha=30^{\circ}$, the injected mass-flow rate in the normal direction to the body surface is increased to $\mathrm{t}_{\max }=0.05$, 0.06 and 0.07 in order to recover the flow asymmetry. Figure 7 shows the results of these tests. Both the TPL contours and the SP-coefficient curves show that the flow is asymmetric and the side force does not vanish. It is seen that the flow asymmetry changes from the right to the left sides and so does the side force. Therefore, it is apparent that normal-injection control loses its effectiveness at high angles of attack for the considered conditions.

\section{Hybrid Beating-Injection Control $\alpha=20^{\circ}-42^{\circ}$}

In this case, the surface-heating control is followed by normal-injection control. Figure 8 shows the results of this study. The control process is applied as follows: First, the whole surface is heated to $T_{1}=5 T_{\infty}$ and the heating control started $a \alpha=20^{\circ}$, where the number of iterations is 12,000 . Next, the angle of attack is increased $4^{\circ}$ until symmetric flow is recovered at the 18,000 iterations. The angle of atrack is then increased $2^{\circ}$ each 1000 iteration until the angle of attack reaches $38^{\circ}$. The flow symmetry has been continuously recovered until the angle of attack of $36^{\circ}$ where a slight flow asymmetry appears in the solution. At $\alpha=38^{\circ}$, the flow asymmetry becomes stronger and the heating control loses its effectiveness for $T_{a}=5 T_{\infty}$. 
At $\alpha=38^{\circ}$, normal-injection control is applied with $\mathrm{m}_{\max }=0.05$ and $\theta=-67.5^{\circ} \rightarrow 67.5^{\circ}$. The angle of attack is then increased $2^{\circ}$ each 3000 iterations. The history of the residual error and the corresponding zero side force is shown in Fig. 8. Figure 9 shows snapshots of the TPL contours and SP-coefficient curves at $\alpha=38^{\circ}$, $40^{\circ}$ and $42^{\circ}$. The TPL contours show asymmetric flows but the SP-coefficient curves show symmetry resulting in zero side force. Thus, it is concluded that a hybrid control of heating and normal injection is very effective in eliminating the side force at very high angles of attack.

\section{Tangential-Injection Control, $\mathrm{m}_{\operatorname{mar}}=\mathbf{0 . 2}$}

In this case, the circular section of the lower portion of the cone has a raidus larger than that of the upper portion. The lower local radius is 1.05 that of the upper one. Flow is injected from the lip of the lower portion in a direction tangent to the surface of the upper portion. The maximum mass flow rate, $\mathrm{m}_{\max }$, is 0.2 and the velocity profile at the lip exit is a parabolic one. Figure 10 shows the grid used for the thin-layer computations. Three grid blocks are used for the present computation. The first block is $121 \times 19 \times 2$, the second is $121 \times 63 \times 2$ and the third is $121 \times 63 \times 2$ all in the circumferential, normal and axial directions, respectively. The tangential flow control starts from the $20^{\circ}$ angle of attack. The computations are carried out until a symmetric flow solution is obcained. Thereafter, the angle of attack is increased to $30^{\circ}$ with a step of $2^{\circ}$. Figure 10 also shows the total pressureloss contours for the controled, symmetric fow solutions at $\alpha=22^{\circ}, 26^{\circ}$ and $30^{\circ}$. It is nociced that as the angle of attack increases the inner shear layers of the controled symmetric primary vortices approach each other and hence flow asymmetry might develop again.

\section{CONCLUDING REMARKS}

The computational solution of the unsteady, compressible, full Navier-Stokes equations has been used to study the effectiveness of two active control methods for asymmetric flows around circular pointed cones. Locallyconical flow assumptions have been used in order to reduce the computational cost of this study by an order of magnitude in comparison with that of three-dimensional flow solutions. The first active control method is applied by injecting air normal to the body surface from ports that are circumferentially distributed within a certain angle. The injected mass-flow rate is either constant or variable. The variable injected mass-flow rate is proportional to the surface-pressure difference between corresponding points on the right and left sides of the body surface. The results of this part of the study show that use of normal injection alone loses its effectiveness at very high angles of attack. The second active control method is applied by heating the body surface either partially or totally. The results of this part of the suddy show that surface-heating control is much more effective in comparison with normal-injection control at very high angles of attack. Next, a hybrid control method which includes surface-heating control followed by normal-injection control is applied. The results of this part of the study shows the superior control effectiveness of the hybrid method Active control using tangential flow injection is very promising. Work is still underway to exploit its effectiveness. Simultaneous application of passive control and the present active control methods is also investigated.

\section{ACKNOWLEDGEMENT}

For the first two authors, this research work has been supported by the NASA Langley Research Center under Grant No. NAG-1-994. The computational resources provided by the NASA Langley Research are approciated.

\section{REFERENCES}

1. Kandil, O. A., Wong, T-C. and Liu, C. H., "Prediction of Steady and Unsteady Asymmetric Vortical Flow Around Cones," AIAA 90-0598, 1990. Also in AIAA Joumal, Vol. 29, No. 12, pp. 1269-1278, 1991.

2. Kandil, O. A., Wong, T-C. and Liu, C. H., "Asymmetric Flow Around Cones with Noncircular Sections," AGARD Symposium on Missile Aerodynamics, AGARD CP No. 493, Friedrickshafen, Germany, pp. 16.1-16.11, 1990.

3. Kandil, O. A., Wong, T-C. and Liu, C. H., "Numerical Simulation of Steady and Unsteady Asymmetric Vortical Flows," ASME Symposium on Non-Steady Fluid Dynamics, FED-Vol. 92, Toronto, Canada, pp. 99108, 1990. To appear in the Journal of Fluids and Structures, 1991.

4. Kandil, O. A., Wong, T-C., Kandil, H., A. and Liu, C. H., "Computation and Control of Asymmetric Vortex Flow Around Circular Cones Using Navier-Stokes Equations," ICAS Paper No. 3.5.3, Vol. 2, Stockholm, Sweden, pp. 883-893, 1990.

5. Kandil, O. A., Wong, T-C., Kandil, H. A. and Liu, C. H., "Thin-Layer and Full Navier-Stokes, LocallyConical and Three-Dimensional Asymmetric Solutions," AIAA 91-0547, 1991.

6. Stahl, W., "Suppression of Asymmetry of Vonex Flow Behind a Circular Cone at High Incidence," Proceedings of the AIAA Atmospheric Flight Mechanics Conference, Boston, MA, AlAA 39-3372-CP, pp. 231-236, 1989.

7. Ng, T. T., "On Leading Edge Vortex and Its Control," Proceedings of the AIAA Atmospheric Flight Mechanics Conference, Boston, MA, AIAA 89-3346CP, pp. 1-15, 1989.

8. Moskovitz, C., Hall, R. and DeJarnette, "Experimental Investigation of a New Device to Control the Asymmetric Flowfield on Forebodies at Large Angles of Attack," AIAA 90-0069, 1990. 
9. Skow, A. M. and Peake, D. J., "Control of the Forebody Vortex Orientation by Asymmetric Air Injection, (Part B) - Detnils of the Flow Structure," AGARD-LS-121, High Angle-of-Attack Aerodynamics, pp. 10.1-10.22, 1982.

10. Ng, T. T., Suarez, C. J. and Malcolm, N., "Forebody Vortex Control Using Slot Blowing," Proceedings of the AIAA 9th Applied Aerodynamics Conference, Baltimore, Maryland, ALAA 91-3254-CP, pp. 412421, 1991.

11. Gittner, M. N. and Chokani, N., “An Experimental Study of the Effects of Aft Blowing on a $3.0 \mathrm{Cal}$ iber Tangent Ogive Body at High Angles of Attack," Proceedings of the AIAA 9th Applied Aerodynamics Conference, Baltimore, Maryland, AIAA 91-3252CP, pp. 390-399, 1991.
12. Font, G. I., Celik, Z. Z. and Roberts, L., “A Numerical and Experimental Sudy of Tangential Jet Blowing Applied to Bodies at High Angles of Attack," Proceedings of the AIAA 9th Applied Acrodynamics Conference, Baltimore, Maryland, AlAA 91-3253CP, pp. 400-411, 1991.

13. Ng, T. T., "Aerodynamic Control of NASP-Type Vehicles Through Vortex Manipulation," AIAA 90-0594, 1990.

14. Tavella, D. A., Schiff, L. B. and Cummings, R. M., "Pneumatic Vortical Flow Control at High Angles of Attack," AIAA 90-0098, 1990.

15. Gee, K., Tavella, D. and Schiff, L. S., "Comptutational Optimization of a Pneumatic Forebody Flow Control Concept" Proceedings of the AIAA 9th Applied Aerodynamics Conference, Baltimore, Maryland, AIAA 91-3249-CP, pp. 370-380, 1991. 
A

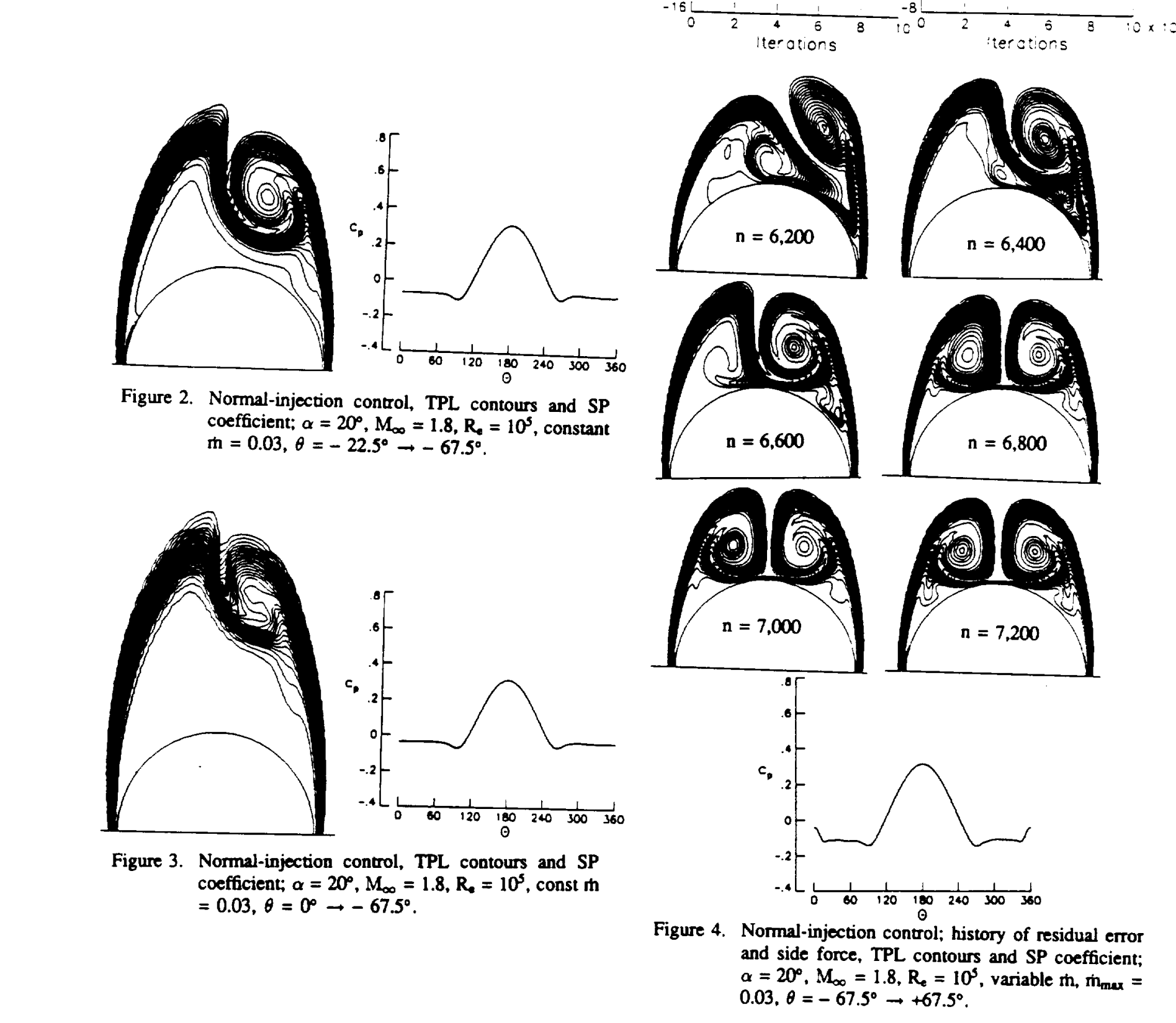



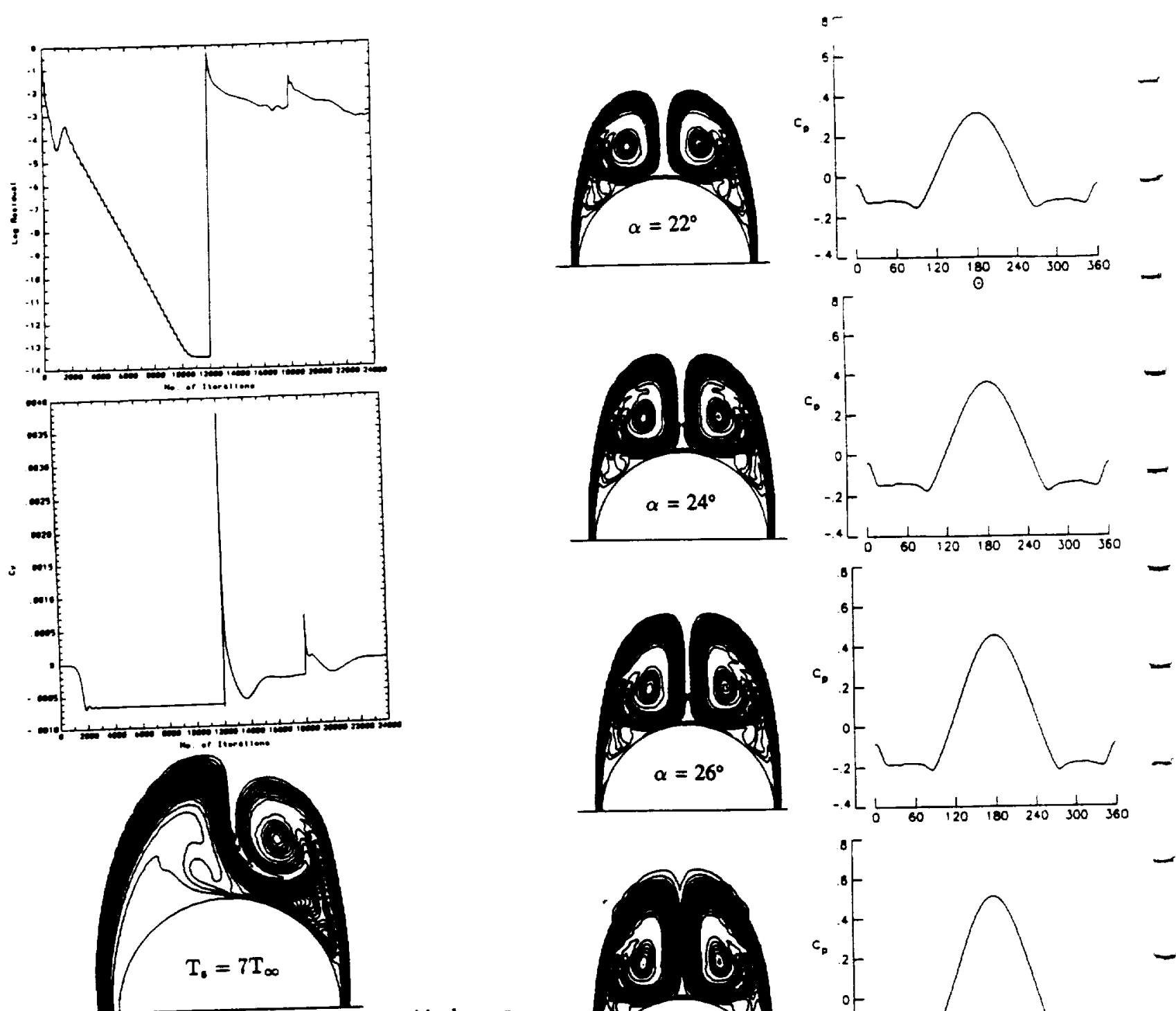

Figure 5. Surface-heating control, history of residual entor and side force and TPL contours; $\alpha=20^{\circ}, \mathrm{M}_{\infty}$ $=1.8, R_{e}=10^{5}, T_{s}=5 \mathrm{~T}_{\infty} \rightarrow 7 \mathrm{~T}_{\infty}, \theta=0 \rightarrow-$ $180^{\circ}$.
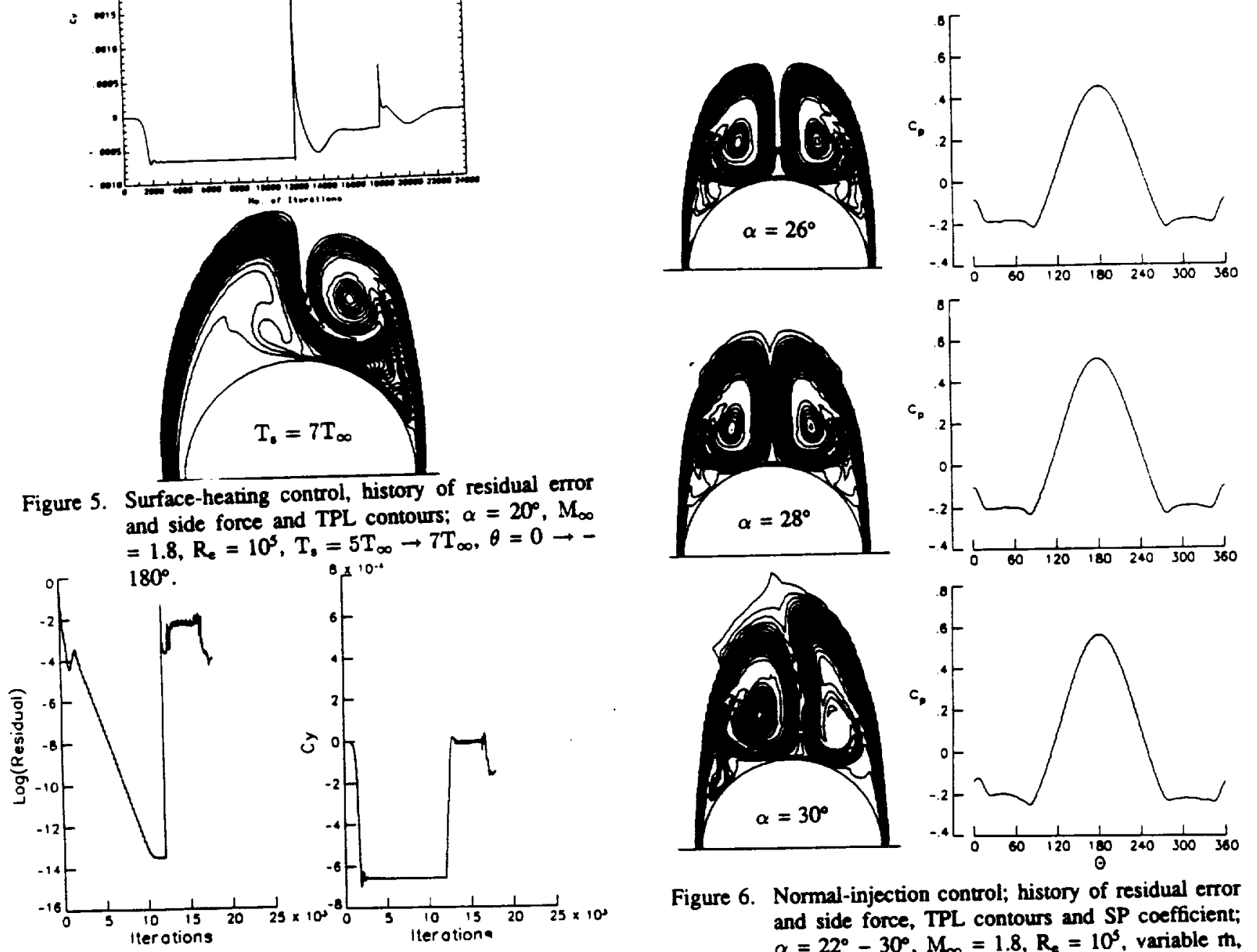

Figure 6. Normal-injection control; history of residual enror and side force, TPL contours and SP coefficient; $\alpha=22^{\circ}-30^{\circ}, M_{\infty}=1.8, R_{e}=10^{5}$, variable th. $m_{\max }=0.03, \theta=-67.5^{\circ}-+67.5^{\circ}$. 


$$
\begin{aligned}
& A \quad M \\
& 2 \quad M \\
& A
\end{aligned}
$$



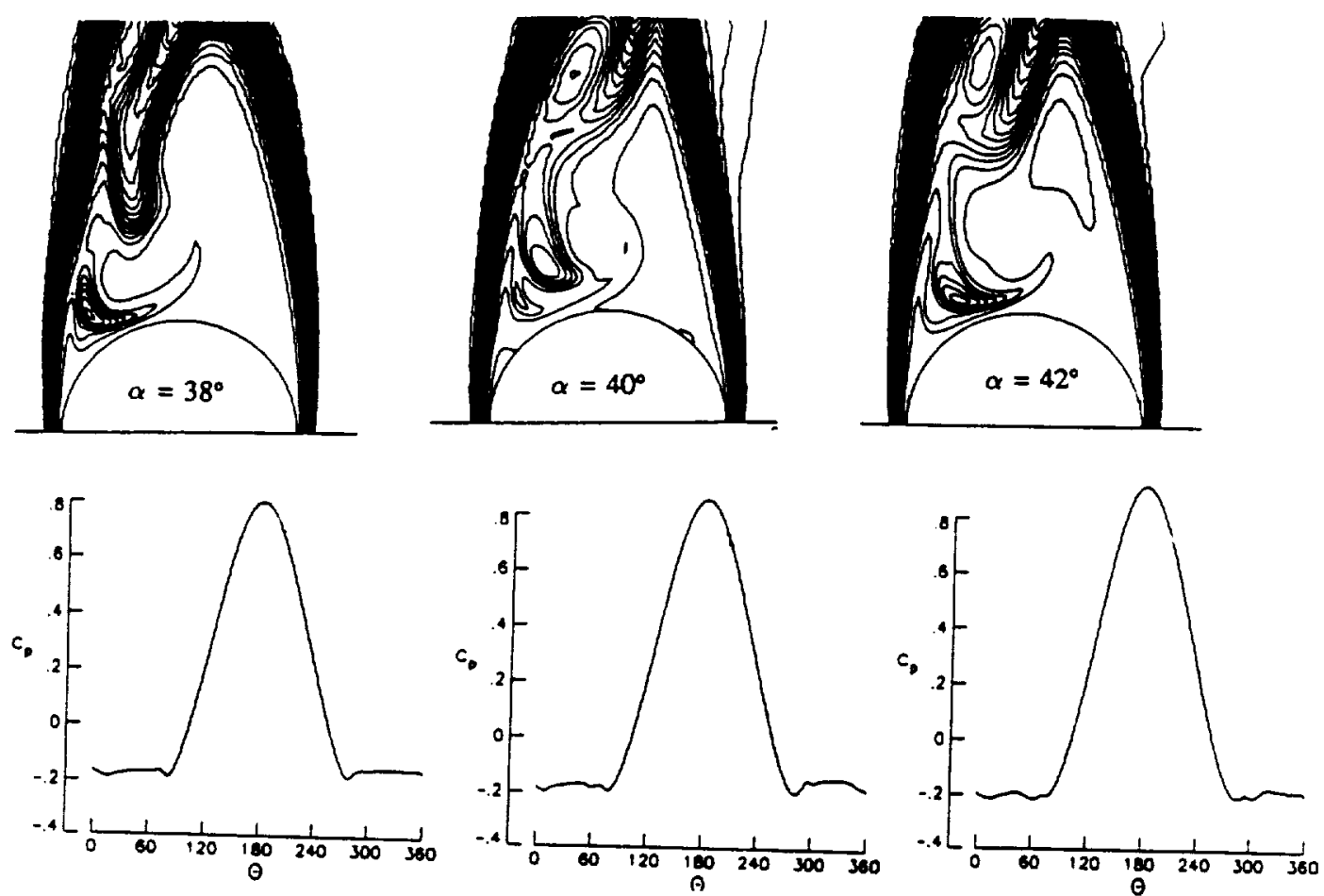

Figure 9. Hybrid heating-injection control (injection part); TPL contours and SP coefficient; $\alpha=38^{\circ}-42^{\circ}$, $M_{\infty}=1.8, R_{0}=10^{5}$, variable $\mathrm{h}, \mathrm{m}_{\max }=0.05, \theta=$ $-67.5^{\circ}-+67.5^{\circ}$.
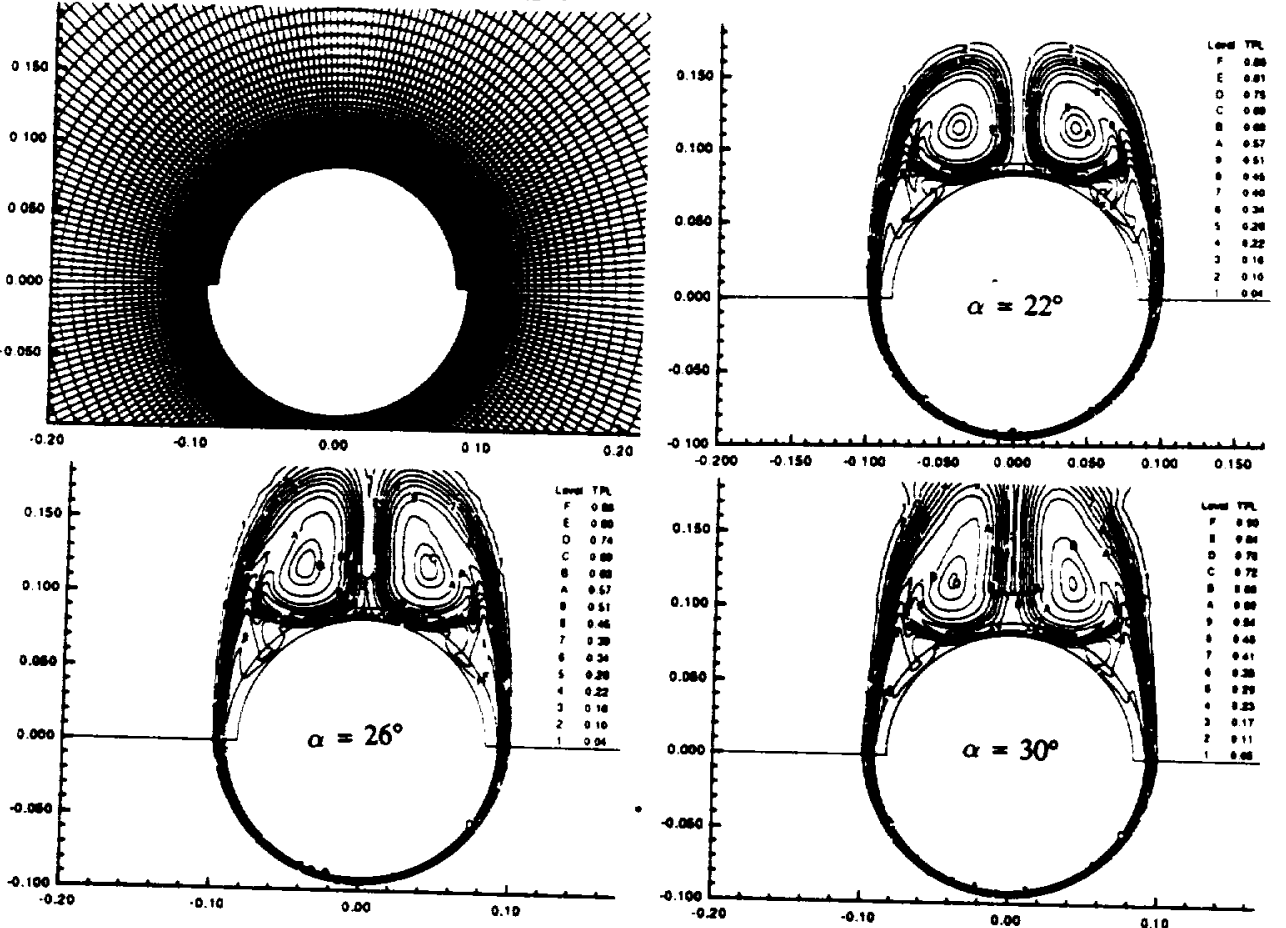

Figure 10. Typical multi-block grid and total pressure-loss contours for the tangential-flow injection control; $\alpha=22^{\circ}, 26^{\circ}, 30^{\circ} ; M_{\infty}=1.8, R_{e}$ $=10^{5}$, parabolic velocity profile at lip exit, $\dot{\mathrm{m}}_{\max }=0.2$ 


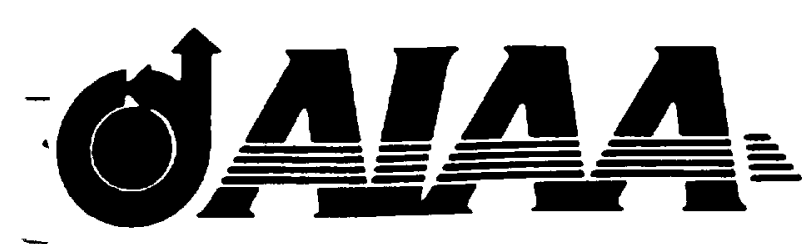

AIAA-92-2601-CP

\section{CRITICAL EFFECTS OF DOWNSTREAM BOUNDARY CONDITIONS ON VORTEX BREAKDOWN}

Osama A. Kandil and Hamdy A. Kandil Old Dominion University, Norfolk, VA 23529

C. H. Liu NASA Langley Research Center, Hampton, VA 23665

\section{AIAA 10th Applied Aerodynamics Conference Palo Alto, CA, June 22-25, 1992}

- For permision to copy or republth, contect the Amorken Instltute of Acronoultes and Astronoutles 370 L'Entent Promenods, S.W., Woinington, D.C. 20024 


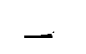




\title{
CRITICAL EFFECTS OF DOWNSTREAM BOUNDARY CONDITIONS ON VORTEX BREAKDOWN
}

\author{
Osama A. Kandil* and Hamdy A. Kandil** \\ Old Dominion University, Norfolk, VA 23529 \\ and \\ C. H. Liu*** \\ NASA Langley Research Center, Hampton, VA 23665
}

\begin{abstract}
The unsteady, compressible, full Navier-Stokes (NS) cquations are used to study the critical effects of the downstream boundary conditions on the supersonic vortex brcakdown. The present study is applied to two supersonic vortex breakdown cases. In the first case, quasiaxisymmetric supersonic swirling flow is considered in a configured circular duct, and in the second case, quasiaxisymmetric supersonic swirling jet, that is issued from a nozzle into a supersonic jet of lower Mach number, is considered. For the configured duct flow, four different types of downstream boundary conditions are used, and for the swirling jet how from the nozzle, two types of downstream boundary conditions are used. The solutions are time accurate which are obtained using an implicit, upwind, flux-difference splitting, finite-volume scheme.
\end{abstract}

\section{INTRODUCTION}

Numerical simulation of vortex breakdown has been focused in most of the existing literature on incompressible flows. Quasi-axisymmctric, vortex-breakdown simulations of incompressiblc swirling hows have been prescnicd by Grabowski and Berger ${ }^{1}$, Hafez, et al. ${ }^{2}$, Salas and Kuruvilla ${ }^{3}$, Menne ${ }^{4}$, Wu and Hwang ${ }^{5}$ and Menne and $\mathrm{Liu}^{6}$. Three-dimensional, vortex-breakdown simulations of incompressible swirling flows have been presented by Spall, et al. ${ }^{7}$, Hanel $^{8}$, and Krause 9.10 . Discussion of the effects of side boundary conditions and downstream boundary conditions on vortex breakdown was presented by Krause in Ref. 10.

Longitudinal vortex/transverse shock-wave interactions are typical applications which appear in transonic and supersonic hows over a strake-wing configuration at modcrate-high angles of attack, at a supersonic inlct injesting a vortex and inside a supersonic combustor where fuel is injected in a swirling jet to enhance fuelair mixing ${ }^{11-13}$. For the strake-wing configuration, vortex brcakdown is undesirable since it results in the stall phenomenon, and hence its occurrence need to be delayed. On the other hand, vortex breakdown for the other two applications is desirable since it enhances mixing and stability of the flame $e^{14,15}$, and hence its occurrence need to be

\footnotetext{
- Profescor and Eminent Scholar, Deparment of Mechanical Engineering and Mechenics, Associste Fellow ALA

- Graduate Rescarch Assistant, Member AUA

- "Group Leader. Theoretical Flow Physica Branch, Areociate Fellow ALAA

Conyright $\odot 1992$ by Osama Kandid. Published by The American Institute of Acronautics and Astronautics, Ine., with permission.
}

controlled for the optimum performance. Unfortunately, the literature lacks this type of analysis with the exception of the preliminary work of Liu, Krause and Menne ${ }^{16}$, Copening and Anderson ${ }^{17}$. Delery, et al, ${ }^{11}$ Kandil and Kandil $^{18}$ and Meadows, Kumer and Hussaini ${ }^{19}$.

The first time-accurate NS solution for a supersonic vortex breakdown was developed by the present authors in Ref. 20. We considered a supersonic quasiaxisymmetric vortex flow in a configured circular duct. The lime-accurate solution of the unsteady, compressible NS equations was obtained using an implicit, upwind, nux-difference splitting finite-volume scheme. A shock wave has been generated near the duct inlet and unsteady vortex breakdown has been predicted behind the shock. The predicted flow was characterized by the evolution, convection and shedding of vortex breakdown bubbles. The Euler equations were also used to solve the same problem. The Euler solution showed larger size and number of vortex-breakdown bubbles in comparison with those of the NS solutions. The time-accurate solution was carricd out for 3,200 times steps which are equivalent to a dimensionless time of 16 . Only one value of Reynolds number of 10,000 was considered in Ref. 20.

In a later paper ${ }^{21}$, we expanded our study of this flow using time-accurate computations of the NS equations with a fine grid in the shock-vortex interaction region and for long computational times. Several issues were addressed in that study. First, we showed the effect of Reynolds number on the temporal evolution and persistence of vortex-breakdown bubbles behind the shock. In that stage of computations, the conditions at the downstream exit were obtained by extrapolating the components of the flowfield vector from the interior cell centers. Although the flow was supersonic over a large portion of the duct exit, subsonic flow existed over a small portion of the exit around the duct centerline. Therefore, selected now cases were computed using a Riemann-invarianttype boundary conditions at subsonic points of the duct exit. Finally, the effect of swirl ratio at the duct inlet was investigated.

In the present paper, we address the problem of specifying the downstream boundary conditions and their critical effects on the supersonic vortex breakdown problem for internal and external flows. For this purpose, the unstcady, compressible, full NS equations are used along with an implicit, upwind, flux-difference splituing, finitevolume scheme for the time-accurate solutions. For the 
intemal how case, supersonic swirling flow in a config. ured duct is considered along with four types of downstream boundary conditions. Keeping the duct geometry and the upstream flow conditions fixed, the exit boundary conditions are varied. The four exit boundary conditions include extrapolation of all the five variables from the intcrior cell centers, specifying the downstream pressure and extrapolating the other now conditions from the intcrior cell centers, specifying the downstream pressure gradient and extrapolating the other four conditions from the interior cell centers, and using a disk of specified radius at the exit section. For the external flow case, supcrsonic swirling jet is issued from a nozzle into a supersonic non-swirling jet of Mach number lower than that of the swirling jet. Two types of downstream boundary conditions are considered. In the first type, extrapolation of all the five variables from the interior cell centers is uscd, while in the second type, the standard Riemanninvariant type boundary condition is used. All the results presented in this paper have been produced using our full NS solver which is known as the "FTNS3D" code. The CRAY-YMP computer of NASA Langley Research Centcr is uscd for the computations.

\section{HIGHLIGHTS OF THE FORMULATION AND COMPUTATIONAL SCHEME}

The conservative, unsteady, compressible, full NavierStokcs equations in terms of time-independent, bodyconformed coordinates $\xi^{1}, \xi^{2}$ and $\xi^{3}$ are used to solve the problem. The equations are given in Ref. 21 and hence they are not shown here. Along with these equations, boundary conditions are specified at the computationaldomain inlet, side wall and downstream exit. The downstrcam cxit boundary conditions will be presented in the nexl section of the computational results. The initial conditions will also be presented in the next section.

The computational scheme used to solve the unsteady, compressible full NS equations is an implicit, upwind, flux-difference splitting, finite-volume scheme. It employs the flux-difference spliting scheme of Roe which is based on the solution of the approximate one-dimensional Riemann problem in each of the three directions. In the Roe scheme, the inviscid flux difference at the interface of a computational cell is split into left and right flux diffcrences. The splitting is accomplished according to the signs of the eigenvalues of the Roe averaged-Jacobian matrix of the inviscid flux at the cell interface. The smooth limiter is used to eliminate oscillations in the shock region. The viscous and heat-flux terms are lincarized and the cross-derivative terms of the viscous Jacobians are dropped in the implicit operator. These terms are differenced using second-order spatially accurate central differencing. The resulting difference equation is approximately factored and is solved in three sweeps in the $\xi^{1} . \xi^{2}$ and $\xi^{3}$ directions. The scheme is used for third. order spatial accuracy and first-order temporal accuracy.
The scheme is coded in the computer program which is called "FTNS3D".

The quasi-axisymmetric solutions are obtained using the three-dimensional code by forcing the flowfield vector to be equal on two axial planes, which are in close proximity of each other.

\section{COMPUTATIONAL RESULTS AND DISCUSSION}

\section{Vortex Breakdown in Configured Circular Duct}

Figure 1 shows a configured circular duct which consists of a short, straight cylindrical part at the inlet which is followed by a short divergent cylindrical part until the axial length of 0.74 . The divergence angle is $6^{\circ}$. The duct radius is then kept constant and a convergent-divergent nozzle with a throat radius of 0.95 is attached. The duct exit radius is 0.98 and its total length is 2.9 . The divergent part of the duct ensures the stability of the formed shock in the inlet region. The configuration of the duct is intended to ensure that the supersonic inflow will become supersonic at the exit. As the computations will show, a small portion of the duct exit near its centerline becomes subsonic at certain times for the specified inflow conditions. This configured duct has also been used by Delery, ct al. " for their Euler equations computations of supersonic vortcx breakdown in an attempt to computationally model an experimental set up.

The NS solver is used to compute all the following flow cases by using a grid of $221 \times 51$ on two axial planes, where 221 points are in the axial direction and 51 points are in the radial direction. In the inlet region up to the 0.74 axial station, 100 grid points are used and the remaining 121 points are used in the remaining part of the duct. The grid is also clustered at the centerline (CL) and the wall. The minimum radial grid size at the $C L$ is 0.002 . The two axial planes are spaced circumferentially at a certain angle so that the aspect ratio of the minimum grid size will be less than 2. The upstream Mach number is kept at 1.75 and the Reynolds number is kept at 100,000. The initial profile for the tangential velocity is given by

$$
\frac{w}{U_{\infty}}=\frac{k_{e}}{r}\left[1-\exp \left(-\frac{r^{2}}{r_{m}^{2}}\right)\right]
$$

where $U_{\infty}=1.74, r_{m}=0.2$ and $k_{\mathrm{e}}=0.1$. The $\max$ imum $\frac{w}{t}$, swirl ratio $\beta$, is at $r=0.224$ and its value is kept at 0.32 . The radial velocity, $v$, at the initial station is set equal to zero and the radial momentum equation is integrated to obtain the initial pressure profile. Finally, the density $\rho$ is obtained from the definition of the speed of sound for the inlet flow. With these compatible set of profiles, the computations are carried out accurately in time with $\Delta t=0.0025$. The wall boundary conditions follow the typical Navier-Stokes solid-boundary conditions. These computations have been carried out on the CRAY YMP of the NASA Langley Research Center. The CPU time is $40 \mu \mathrm{s} / \mathrm{grid}$ point/iteration for the NS calculation.
$-$
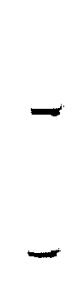

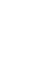$$
-
$$$$
-
$$

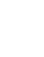$$
-
$$

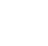$$
\text { - }
$$
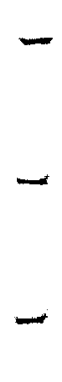
$\mathrm{Ncxt}$, we present the results of the computational study of this case which covers four types of exit boundary conditions.

\subsection{Extrapolation from Interior Cell Centers}

Figure 2 shows snapshots of the streamlines and Mach contours for this case. The downstream boundary conditions are obtained by extrapolating all the flow variables from the cell centers at the exit. The streamlines snapshots show multi-bubble vortex breakdown evolution, convection, merging and shedding. The time-accurate integration was carried out up to $t=200$ and the solution showed periodic multi-frequency cycles of vortexbreakdown bubbles ${ }^{21}$. An example of the merging of vortex breakdown bubbles of same sign of voricity is shown at $t=17$. An example of convection and shedding of vortex breakdown bubbles is shown at $t=25$. Comparing the streamlines solutions at $t=25$ and $t=$ 89 , it is scen that the solutions are almost the same which conclusivcly show that the breakdown process is periodic. The Mach-contours show the dynamics of inlet shock motion. In the time range of $t=3-8$, the inlet shock moves upstream toward the inlet and its central portion exists outside the inlet section at $t=8$. In the time range of $t=8-25$, the inlet shock moves downstream with corresponding evolution, convection, merging and shedding of brcakdown bubbles. In the time range of $t=25-45$, the inlet shock maintains its motion in the downstream direction at a slower rate than before, while another shock, which is downstream of the inlet shock, appears and also moves in the downstream direction. The evolution, convecuion and shedding slowly continues until $t=66$. In the time range of $t=66-78$, the downstream shock disappears and a large vortex-breakdown bubble appears and moves upstrcam. This motion of the bubble is accompanicd by upstream motion of the inlet shock $(t=78)$. Later the inlet shock again moves in the downstream direction and the process is repeated. An animation movie has been produced for the lotal dimensionless time of $t$ $=200$. Figure 3 shows a blow-up of two snapshots of the strcamlines solutions at $t=84$ and $t=87$. At $t=84$, we recognize five vortex breakdown bubbles which spatially alternate in their sign of vontex strength. It is also noticed that six stagnation points exist at the axis. At t $=87$, we recognize seven vortex breakdown bubbles and scven stagnation points. The figure shows the merging of two bubbles of same vorticity sign.

\subsection{Riemann Invariant Boundary Conditions with $p_{b}=p_{\infty}$}

In this case, the back pressure at the subsonic points of the duct exist, $p_{b}$, is specified to be equal to $p_{\infty}$ and the other four variables are extrapolated form the interior cell centers. The computations have repeated on the same grid and for the same flow conditions as that of the previous case. Figure 4 shows snapshots of the streamlines and Mach contours of the solution. Comparing the present solution with the previous case (Fig. 2), it is seen that the two solutions are the same until $t=35$. Thereafter, for $\mathrm{t}>35$, the inlet shock continuously moves in the downstream direction with the vortex-breakdown bubbles moving ahead of the shock. The shock and vortex bubbles are shed and disappears from the duct at advanced levels of time. The breakdown mode is termed as "a transient multi-bubble vortex breakdown." The reason behind disappearance of the shock-vortex-breakdown-bubble system is that the back pressure is so low that it cannot support the inlet shock keeping it in the inlet region. Moreover, the Riemann-invariant type conditions at subsonic points let the downstream effects propagate upstream as time increases.

\subsection{Riemann Invariant Boundary Conditions with $p=2 p_{\infty}$}

In this case, the back pressure at the subsonic points of the duct exit is specified to be $p_{b}=2 p_{\infty}$ and the other four variables are extrapolated from the interior cell centers. Figure 5 shows snapshots of the streamlines and Mach contours of the solution. Comparing the present solution with the solution of the first case (Fig. 2), it is seen that the two solutions are similar with the exception that the present solution lags that of the first case in time. The reason behind this behavior is that the back pressure $p_{b}$ is larger than that of the first case. Moreover, the Riemann invariant type conditions at subsonic points let the downstream effects propagate upstream as time increases. The existence of the large back pressure which is felt upstream supports the inlet shock and keeps it in the inlet region.

\section{I.4. Riemann Invariant Boundary Conditions with $\frac{\partial p}{\partial z}=$ constant}

In this case, the back pressure is obtained from the condition $\frac{\partial p}{\partial x}=$ constant at the subsonic points of the duct exit. The other flow variables are extrapolated from the interior cell centers. Figure 6 shows snapshots of the streamlines and Mach contours of the solution. Comparing the present solution with the solution of the first case (Fig. 2), it is seen that the two solutions are similar until $t=22$. Thereafter, for $t>22$, the inlet shock continuously moves in the downstream direction with the vortexbreakdown bubbles moving ahead of the shock. Again, as in the case of Fig. 4, the shock and vortex bubbles are shed and disappear from the duct at advanced levels of time. The breakdown is termed as "a transient multibubble vortex breakdown." The reason behind disappearance of the shock-vortex-breakdown-bubble system is that the back pressure obtained from $\frac{\partial_{p}}{\partial x}=$ constant condition is so low that it cannot support the inlet shock keeping it in the inlet region. Moreover, the Riemann-invariant type conditions at subsonic points let the downstream effects propagate upstream as time increases. 


\section{I.5. Placing a Disk at the Exit with $r=0.333$}

In this case, a circular solid disk of radius $r=0.333$ is placed at the duct exit and solid-boundary conditions are applied on the disk surface. For the remaining portion of the exit, the boundary conditions are obtained by cxtrapolation from the interior cell centers. Figure 7 shows snapshots of the streamlines and Mach contours of the solution. It is noticed from the Mach contours that most of the exit points are subsonic. The streamlines show that the shock-vortex-breakown-bubble system first appears behind the duct exit. Thereafter, they move upstreams until the inlet shock moves outside of the inlet. The vortex-breakdown bubbles are then locked between the shock outside of the inlet and the circular disk at the exit.

\section{Supersonic Swirling Jet from a Nozzle}

Figure 8 shows the computational domain and a typical grid for this external flow case. The dimensions of the computational domain is $7 \times 3.5$ in the axial and radial directions, respectively. The grid is $221 \times 51 \times 2$ points in the axial, radial and tangential directions, respectively. The grid is clustered at the nozzle exit $(x=0.0)$ and at the domain centerline. The dimensionless nozzle radius is unity, where a supersonic jet of $M_{\infty}=3.0$ is issued, and outside the nozzle another jet is issued at $M_{\infty}=2.0$. The freestream Reynolds number is 296,000 .

Figure 9 shows the inlet flow profiles of the axial velocity, swirl velocity, radial velocity, pressure and density, which are taken from the experimental data of Ref. 12. The initial profiles are used as quasiaxisymmetric profiles for the present computations. On the cylindrical boundary (side wall) of the flow at $r=3.5$, freestream conditions are imposed corresponding to $M_{\alpha}$, $=2.0$. The initial conditions in the computational domain are also taken as those corresponding to the freestream conditions at $M_{\alpha}=2.0$. The problem is solved using two types of exit boundary conditions at $x=7.0$; first extrapolation of all five variables from the interior cell center and second using the Riemnn-invariant-type boundary conditions.

\section{II.1. Extrapolation from Interior Cell Centers}

Figure 10 shows snapshots of streamlines and Mach contours of the solution. The streamlines show multibubble breakdown at the early levels of time. These bubblcs develop due to the shock system formed at the nozzle cxit in the vicinity of the centerline. It is noticed that a strong portion of the shock exists at the centerline which splits into two oblique shocks, one is a weak shock and the other is a strong shock. Behind the strong shock, the vortex breakdown bubbles exist. Thereafter, for $t>5$, the oblique shocks move slowly in the downstream direction and breakdown the vortex bubble stays in its place. At $t$ $>55$, the shock system moves upstream and so does the vortex breakdown bubble. The slow motion of the shock system and the vortex breakdown bubble continues back and forth between these two locations. No vonex shedding has been captured during the computations of this case. It is also noticed that most of the exit points are continuously supersonic and hence no downstream effects exist with the exception of a very thin-layer around the centerline.

\section{II.2. Riemann Invariant Boundary Conditions}

Next, the boundary conditions at the exit are replaced by using the Riemann-invariant-type boundary conditions with $p_{b}=p_{\infty}$ at the subsonic points. Figure 11 shows snapshots of the streamlines and Mach contours of the solution. By comparing the present solution with the previous case of Fig. 10, we see that there is very slight effect of the present boundary condition on the solution. This is understood since the subsonic region at the exit is very small and moreover, the exit boundary is far from the nozzle exit.

Figure 12 shows a blow-up of the Mach contours at $t$ $=55$ for the llow case of Fig. 10. The shock system near the nozzle exit is clearly seen.

\section{Concluding Remarks}

The unsteady, compressible full Navier-Stokes equations are used to study the critical effects of the downstream boundary conditions on the supersonic vortex breakdown. In the present study, two supersonic swirling flow cases are considered. The first one is for a supersonic swirling flow in a configured circular duct, where four types of exit boundary conditions are used. The second one is for a supersonic swirling jet issued from a nozzle into another supersonic jet of lower Mach number. This flow case is a computational simulation to the experimental case of Ref. 12 .

In the first flow case, we have shown that the downstream exit boundary conditions produce substantial changes in the vortex breakdown modes, particularly at advanced time levels. This is due to the effect of the subsonic downstream points on the flow upstream. In the second flow case, we have shown that the downstream exit boundary conditions do not produce substantial changes in the vortex breakdown modes. This is due to the very thin subsonic region at the exit and the large distance of the downstream exit from the nozzle exit.

\section{Acknowledgement}

For the first two authors, this research work has been supported by NASA Langley Research Center under Grant No. NAG-1-994. 


\section{References}

1. Grabowski, W. J. and Berger, S. A., "Solutions of the Navier-Stokes Equations for Vortex Breakdown," Journal of Fluid Mechanics, Vol. 75, Part 3, 1976, pp. 525-544.

2. Hafez, M., Kuruvila, G. and Salas, M. D., "Numerical Study of Vortex Breakdown," Joumal of Applied Numerical Mathematics, No. 2, 1987, pp. 291-302.

3. Salas, M. D. and Kuruvila, G.. "Vortex Breakdown Simulation" A Circumspect Study of the Steady, Laminar, Axisymmetric Model," Computers and Fluids, Vol. 17, No. 1, 1989, pp. 247-262.

4. Menne, S., "Vortex Breakdown in an Axisymmetric Flow," AIAA 88-0506, January 1988.

5. Wu, J. C. and Hwang, S., "Computational Study of Vortex Breakdown in Circular Tube," AIAA 91-1820, June 1991.

6. Menne, S. and Liu, C. H., "Numerical Simulation of a Three-Dimensional Vortex Breakdown," Z. Flugwiss. Weltraumforsch. 14, 1990, pp. 301-308.

7. Spall, R. E., Gatski, T. B. and Ash, R. L., "The Structur and Dynamics of Bubble-Type Vortex Breakdown," Proc. R. Soc., London, A429, 1990, pp. 613637.

8. Brever, M. and Hanel, D., "Solution of the 3-D Incompressible Navier-Stokes Equations for the Simulation of Vorex Breakdown," Eight GAMM Conference, Delft, Netherlands, September 27-29, 1989.

9. Krause, E., "Vortex Breakdown: Physical Issues and Computational Simulation," Third International Congress of Fluid Mechanics, Cairo, Egypt, Janury 1990, Vol. 1, pp. 335-344.

10. Krause, E., "The Solution of the Problem of Vortex Breakdown," Invited paper, Intermational Conference on Numerical Methods for Fluid Dynamics, Oxford, England, June 1990.
11. Delery, J., Horowitz, E., Leuchter, O. and Solignac, J. L., "Fundamental Studies of Vortex Flows," La Recherche Aerospatiale, No. 1984-2, 1984, pp. 1-24.

12. Metwally, O., Setules, G. and Horstman, C., "An Experimentally Study of Shock Wave/Vortex Interaction," AIAA 89-0082, January 1989.

13. Cutler, A.D. and Levey, B. S., "Vortex Breakdown in a Supersonic Jet," AIAA 91-1815, June 1991.

14. Rhode, D. L., Lilley, D. G. and McLaughlin, D. K., "On the Prediction of Swirling Flowfields Found in Axisymmetric Combustor Geometries," Transactions of ASME, Vol. 104, September 1982, pp. 378-384.

15. Altegeld, H., Jones, W. P. and Wilhelmi, "Velocity Measurements in a Confined Swirl Driven Recirculating Flow," Experiments in Fluids, Springer Verlag. Vol. 1, 1983, pp. 73-78.

16. Liu, C. H., Krause, E. and Menne, S., "Admissible Upstream Conditions for Slender Compressible Vortices," AIAA 86-1093, 1986.

17. Copening, G. and Anderson, J., "Numerical Solutions to Three-Dimensional Shock/Vortex Interaction at Hypersonic Speeds," AIAA 89-0674, January 1989.

18. Kandil, O. A. and Kandil, H. A., "Computation of Compressible Quasi-Axisymmetric Slender Vortex Flow and Breakdown," IMACS ist International Conference on Computational Physics, University of Colorado, Boulder, June 1990, pp. 46-51. Also in Computer Physics Communications, Vol. 65, NorthHolland, March 1991, pp. 164-172.

19. Meadows, K., Kumar, A. and Hussaini, M., “A Computational Study on the Interaction Between a Vortex and a Shock Wave," AIAA 89-1043, April 1989."

20. Kandil, O. A., Kandil, H.A. and Liu, C. H., "Computation of Steady and Unsteady Compressible QuasiAxisymmetric Vonex Flow and Breakdown," AIAA 91-0752, January 1991.

21. Kandil, O. A., Kandil, H. A. and Liu, C. H., "Supersonic Quasi-Axisymmetric Vortex Breakdown," AIAA 91-3311-CP, September 1991, pp. 851-863. 
$r_{\text {th }}=0.95, \mathrm{sex}_{\mathrm{ex}}=0.98$

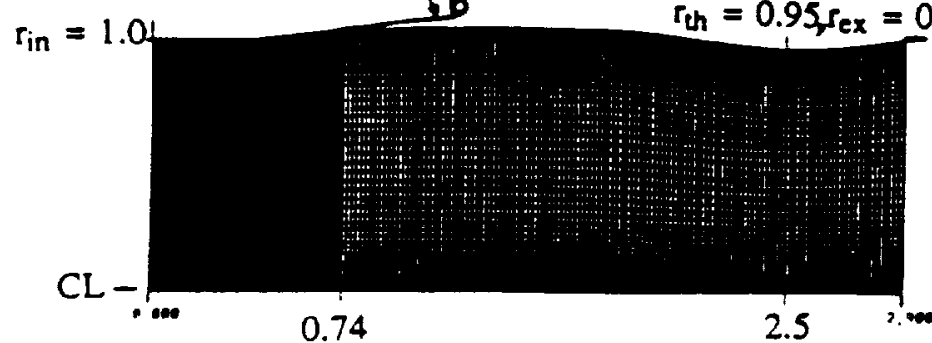

Figure 1. Typical grid for a supersonic configured circular duct; $221 \times 51 \times 2$

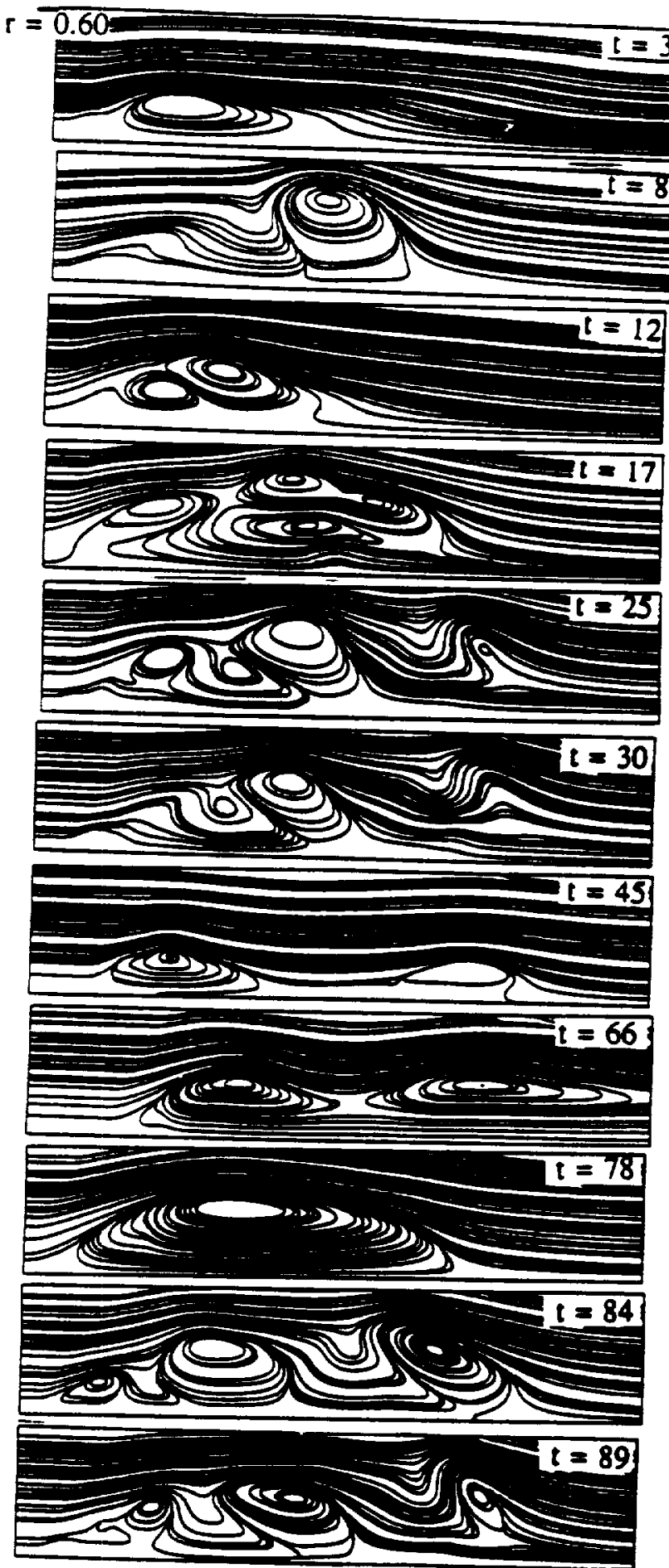

Figure 2. Streamlines and Mach contours for a swirling flow with periodic multibubble, multifrequency vortex breakdown, extrapolation from interior.
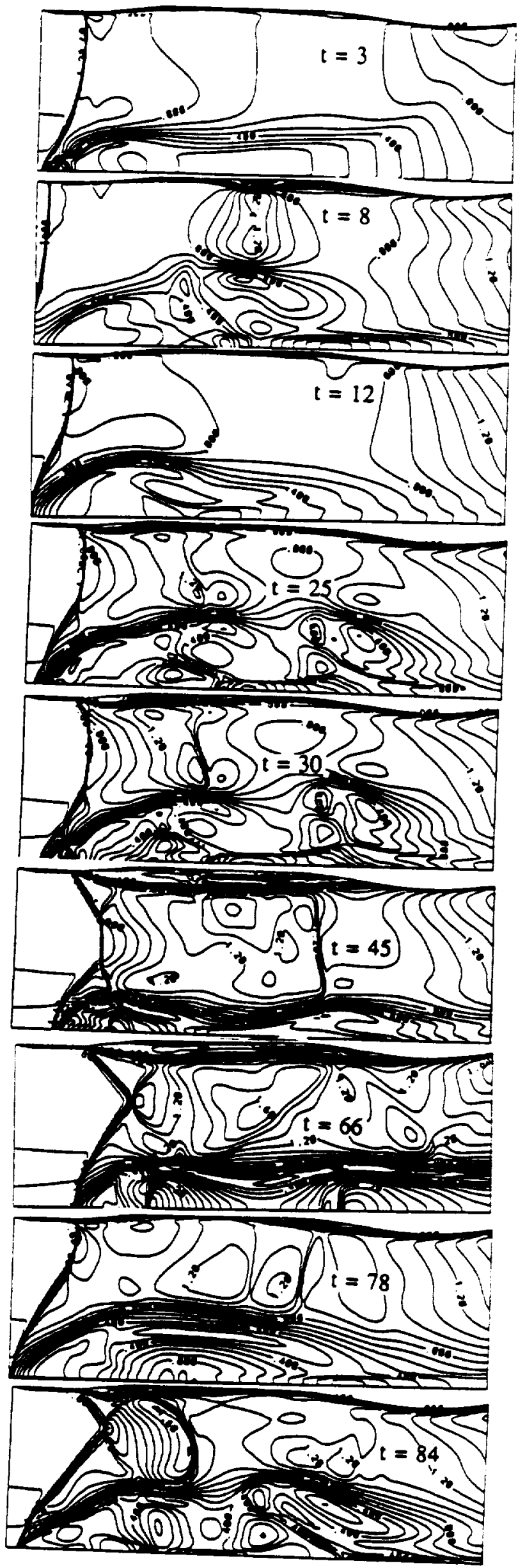

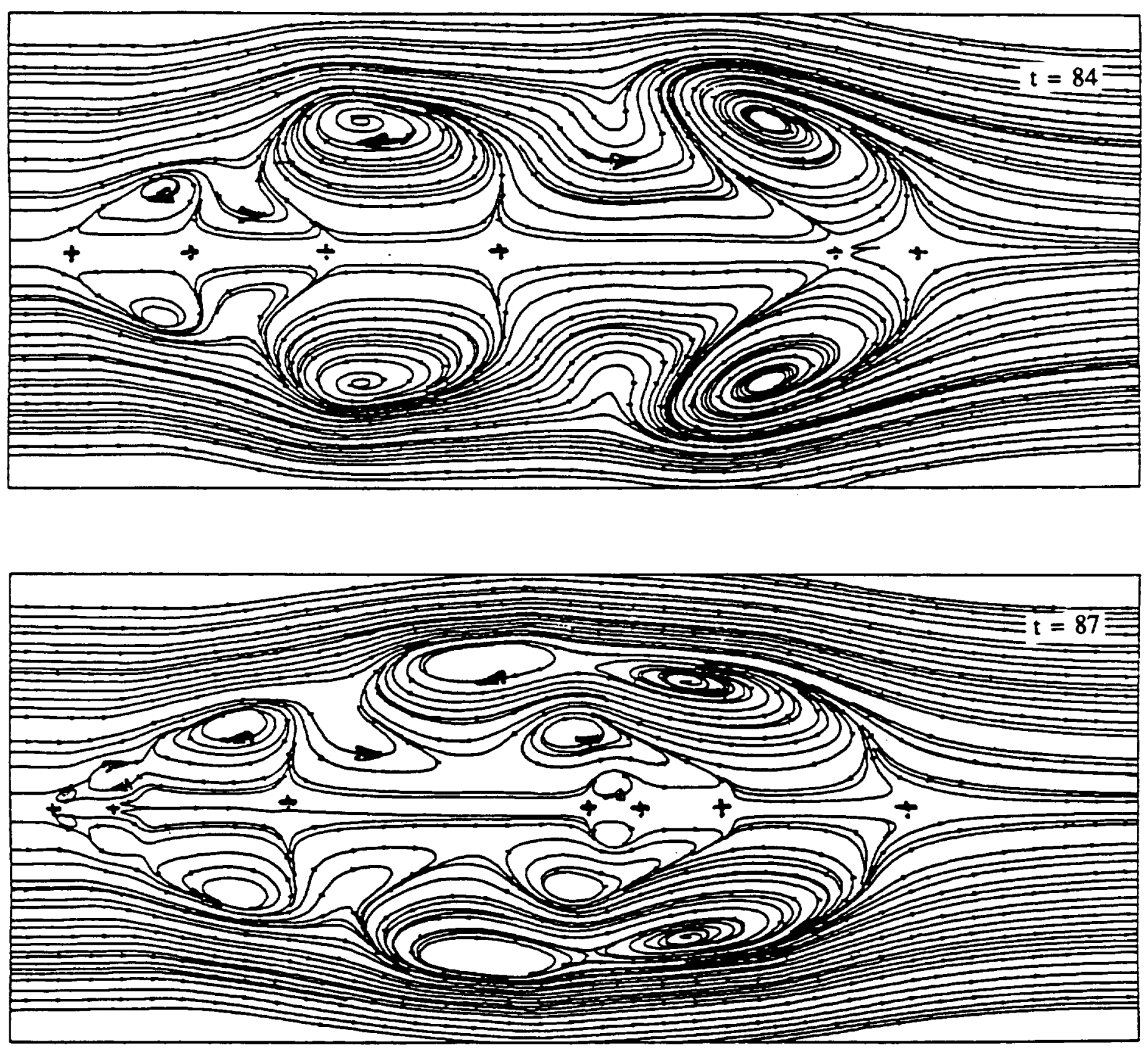

Figure 3. Blow-up of streamlines of periodic multibubble, multifrequency vortex breakdown, extrapolation from interior B.C. 
$r=0.6$
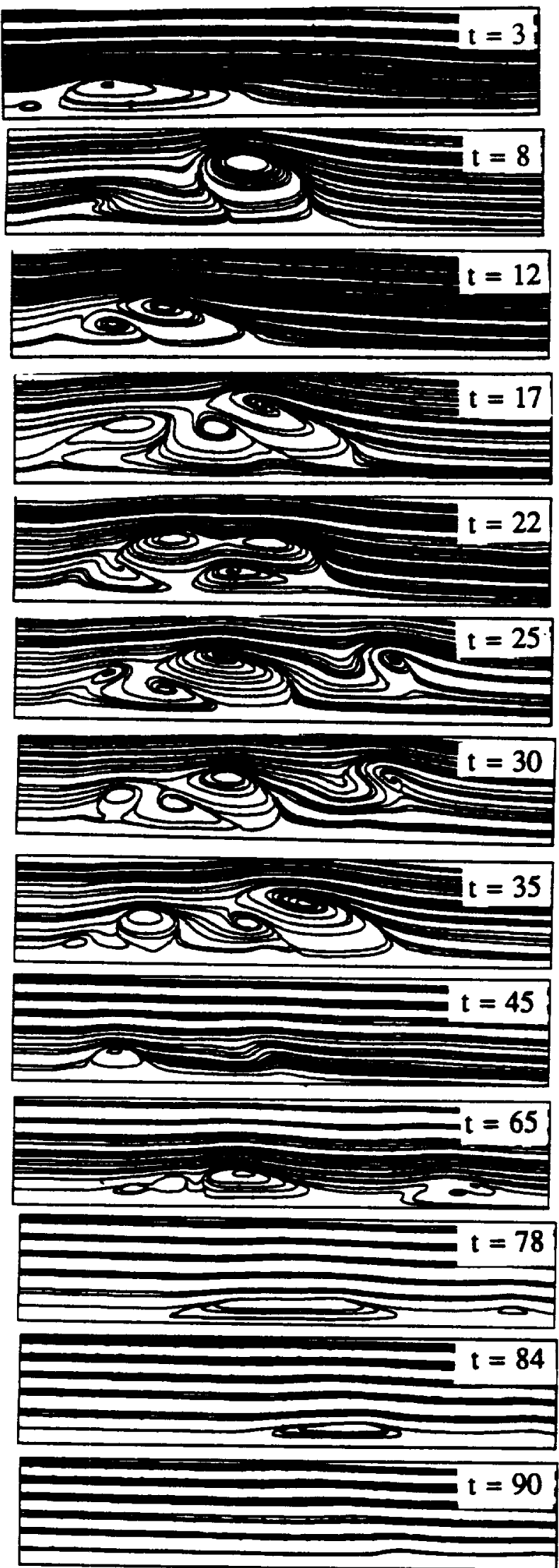

Figure 4. Streamlines and Mach contours for a swirling flow with transient multibubble vortex breakdown, $P_{b}=P_{\infty}$, Riemann invariant B.C.
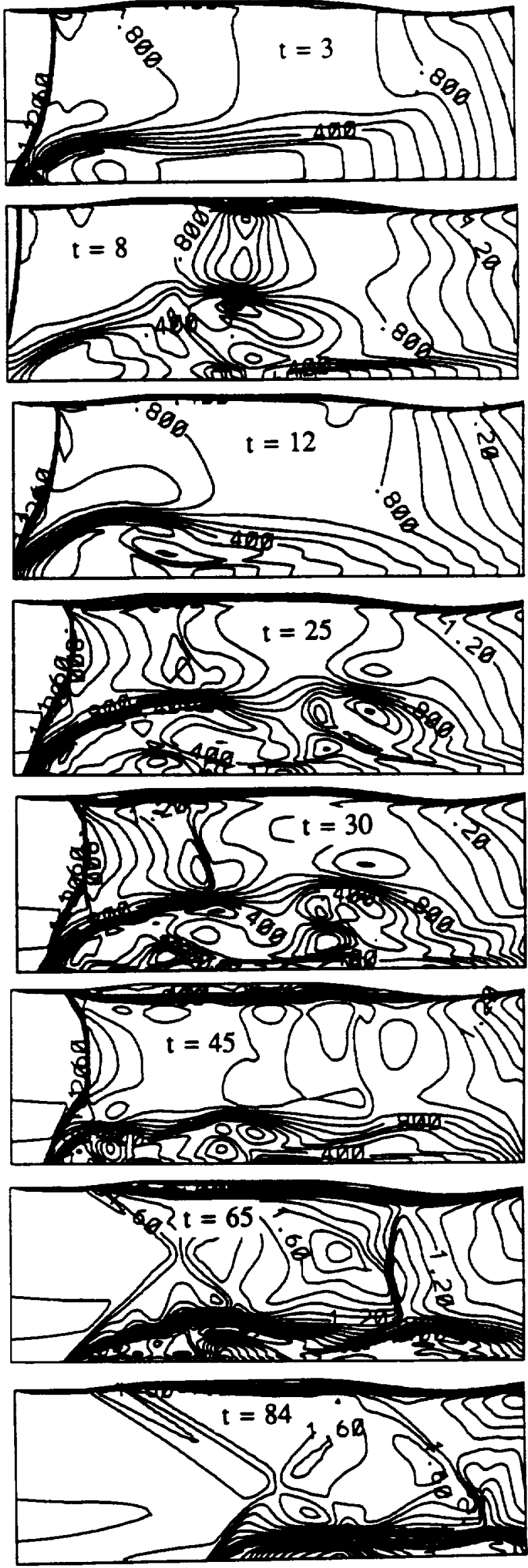


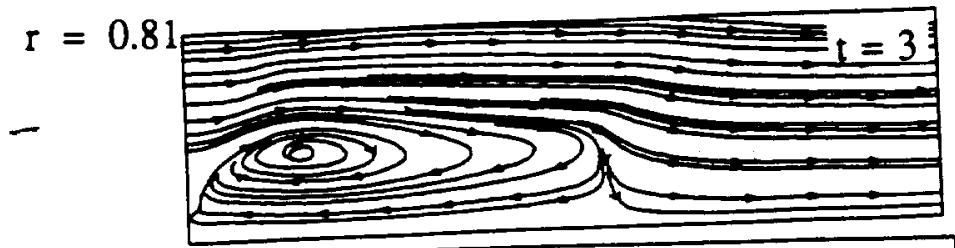

$-\quad 08$ $\Longrightarrow$ $\Longrightarrow$ 00 $\Longrightarrow \mathrm{t}$ $\Longrightarrow \Longrightarrow$ $\Longrightarrow 20$

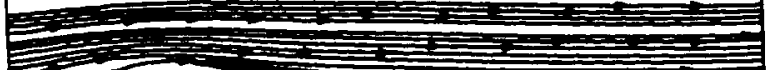
02020
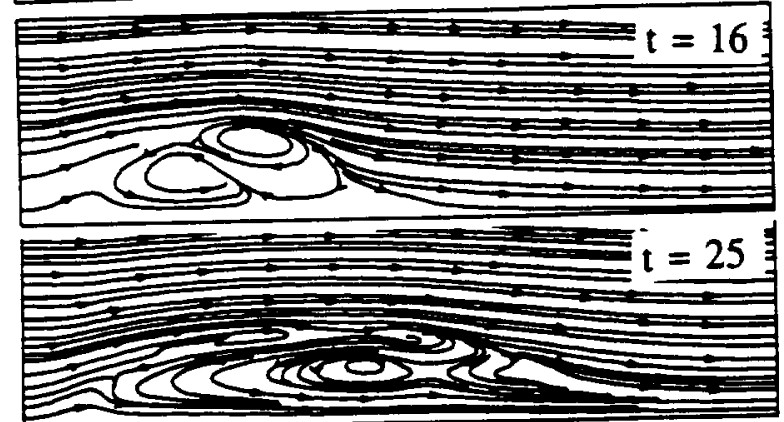

$\Longrightarrow \mathrm{C}=30$ $\Longrightarrow 200=20$

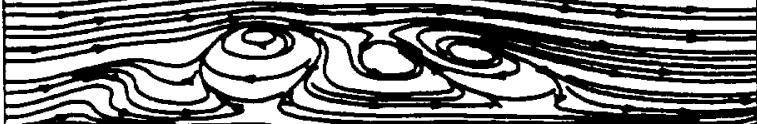

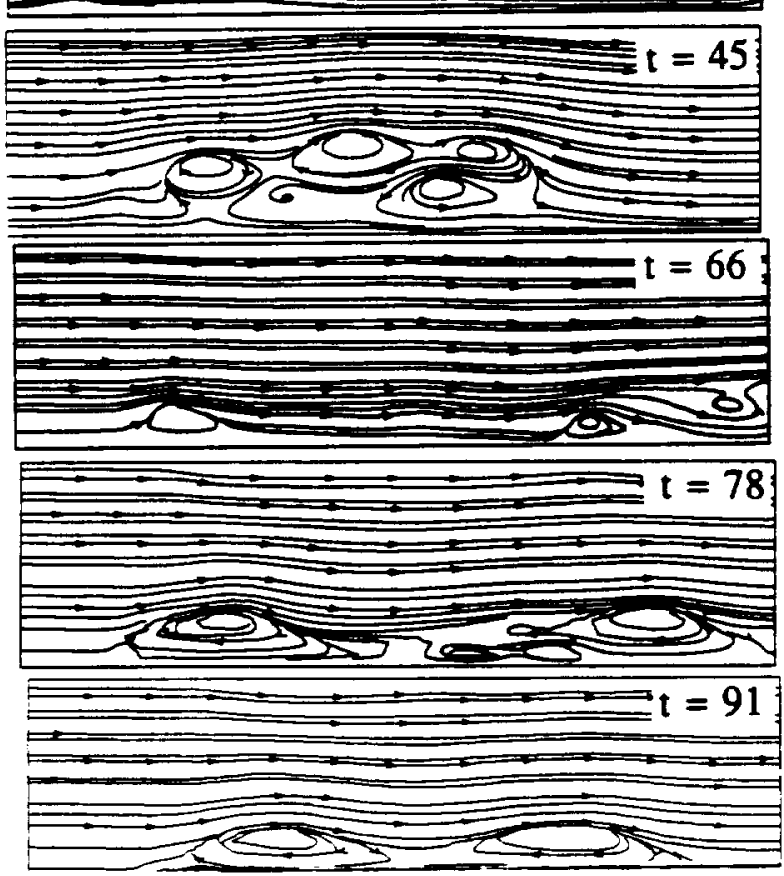

Figure 5. Streamlines and Mach contours for a swirling flow with periodic multibubble, multifrequency vortex breakdown, $P_{b}=2 P_{\infty}$, Riemann invariant B.C.
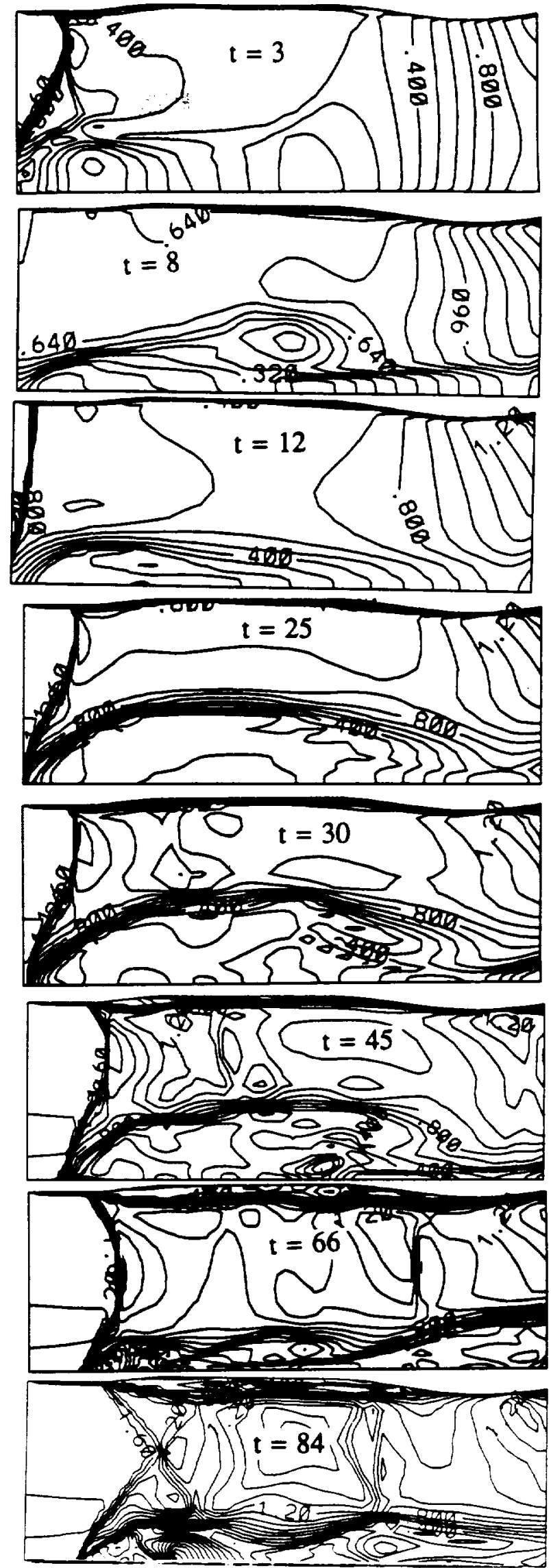

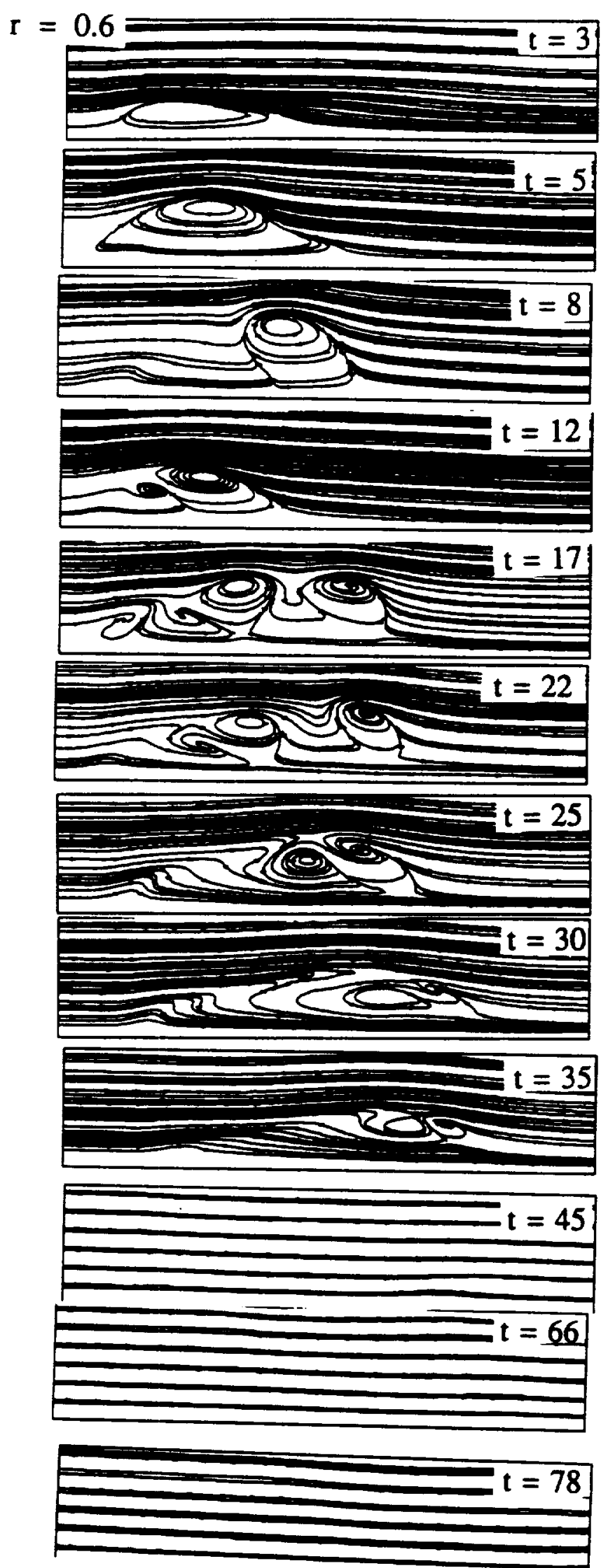

Figure 6. Streamlines and Mach contours for a swirting flow with transient multibubble vortex breakdown, $\frac{\partial p}{\partial x}=c$, Riemann invariant B.C.
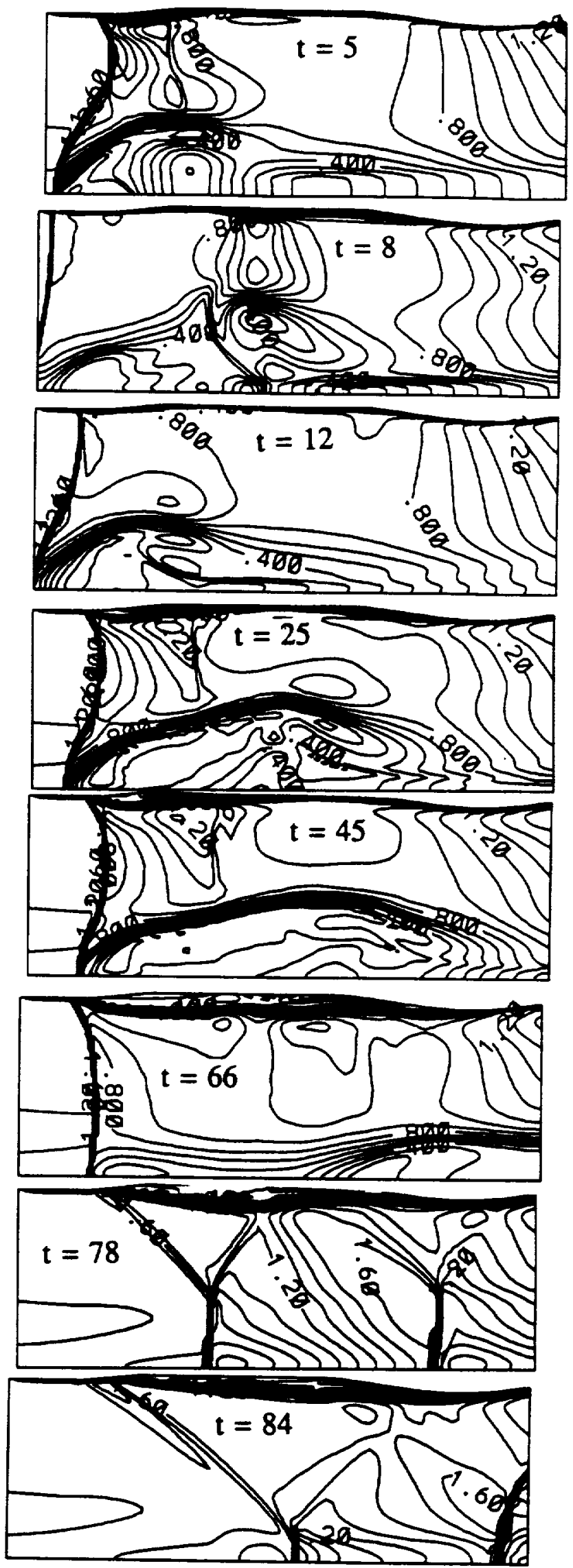

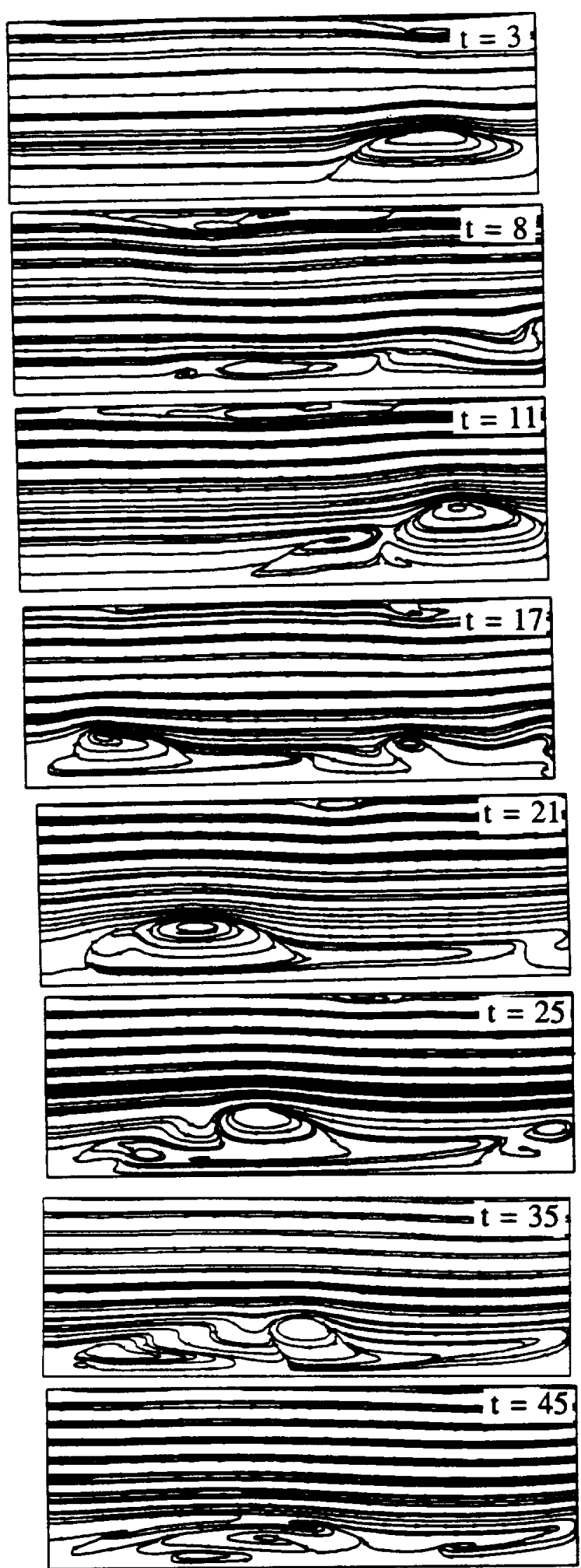
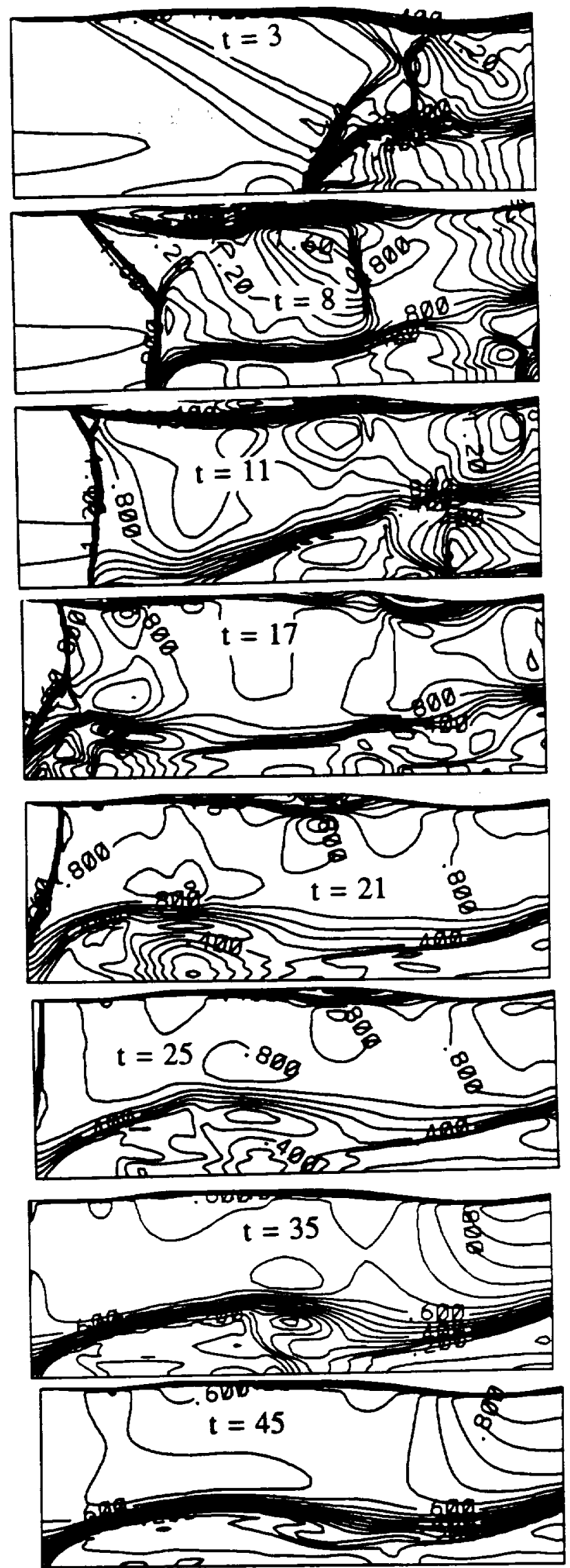

Figure 7. Streamlines and Mach contours for a swirling flow with quasi-steady multibubble vortex breakdown, downstream disk of $r=0.333$. 


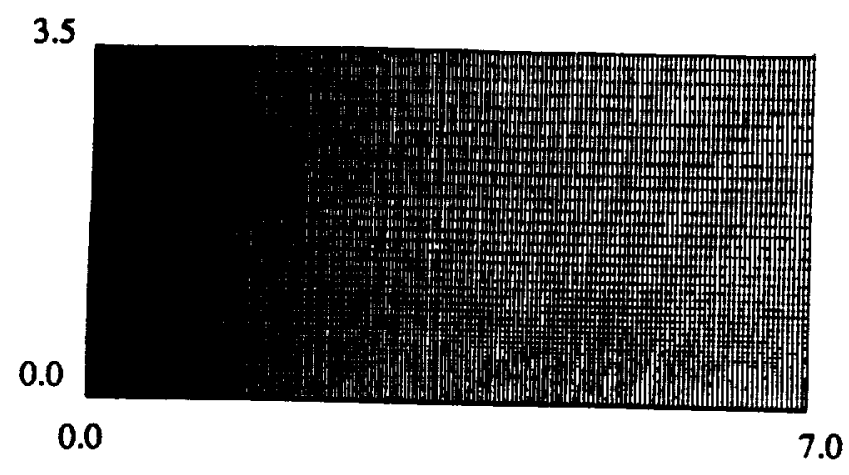

Figure 8. Typical grid for supersonic swirling jet from a nozzle, $221 \times 51 \times 2$
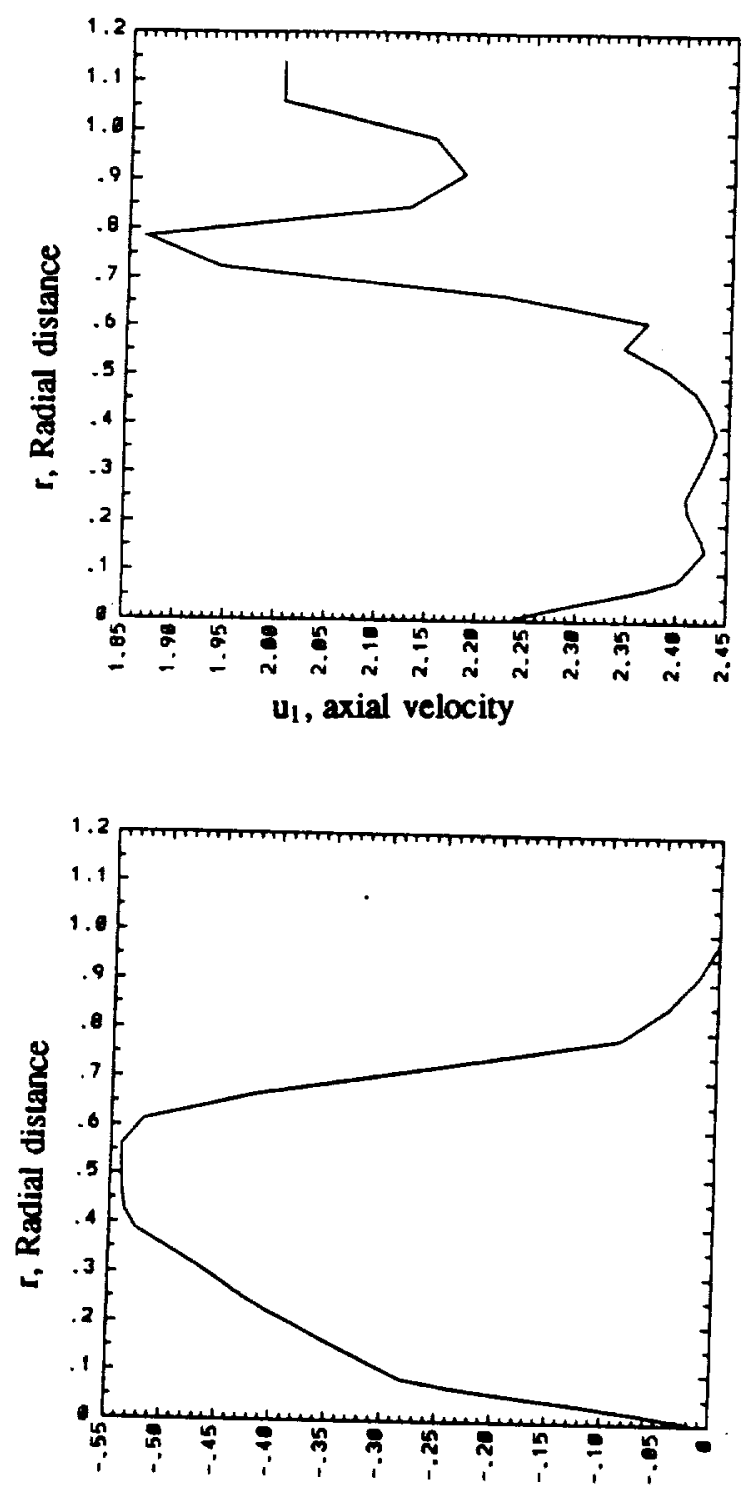

$u_{2}$, Tangential velocity
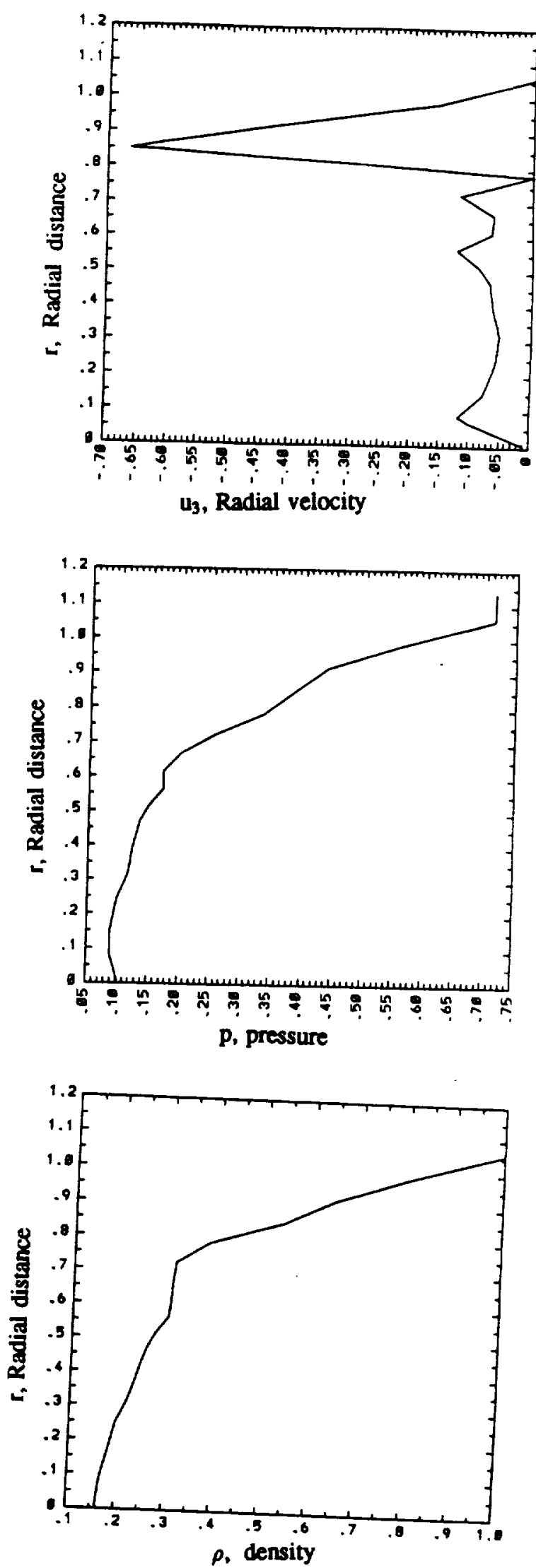

Figure 9. Quasi-axisymmetric flow profiles at $x=0.0$ for supersonic swirling jet from a nozzle. 
$r=0.695$
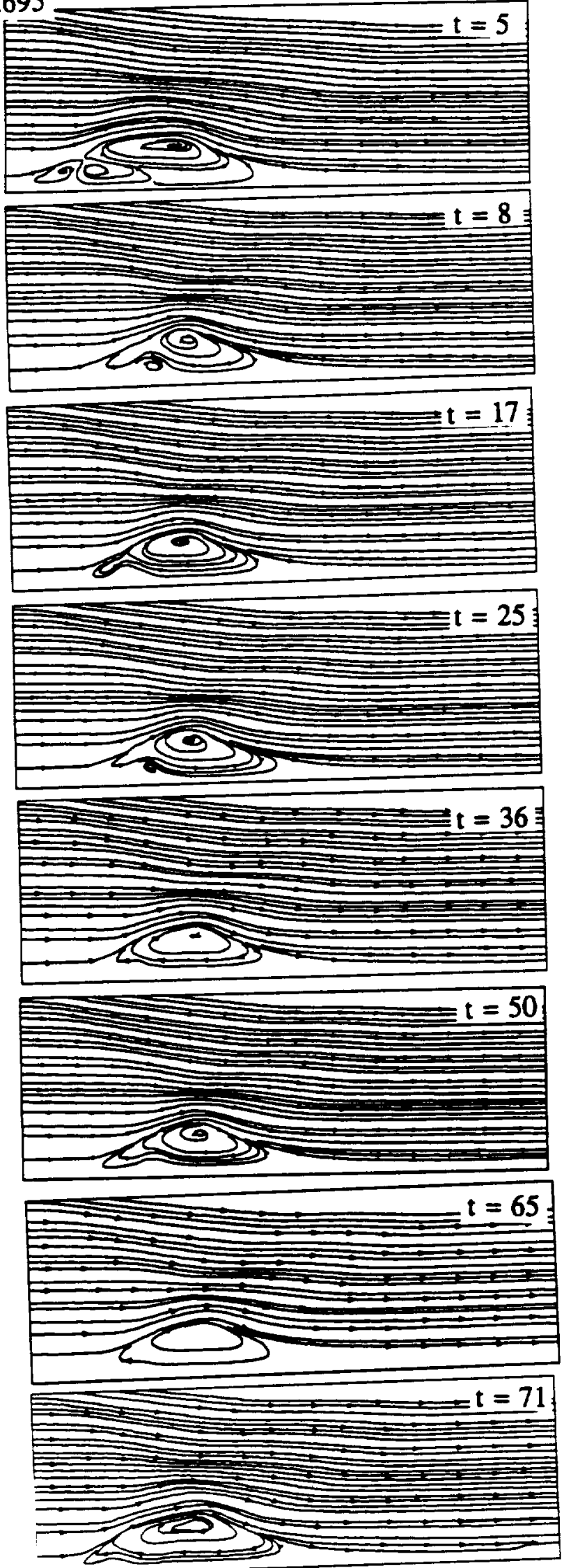
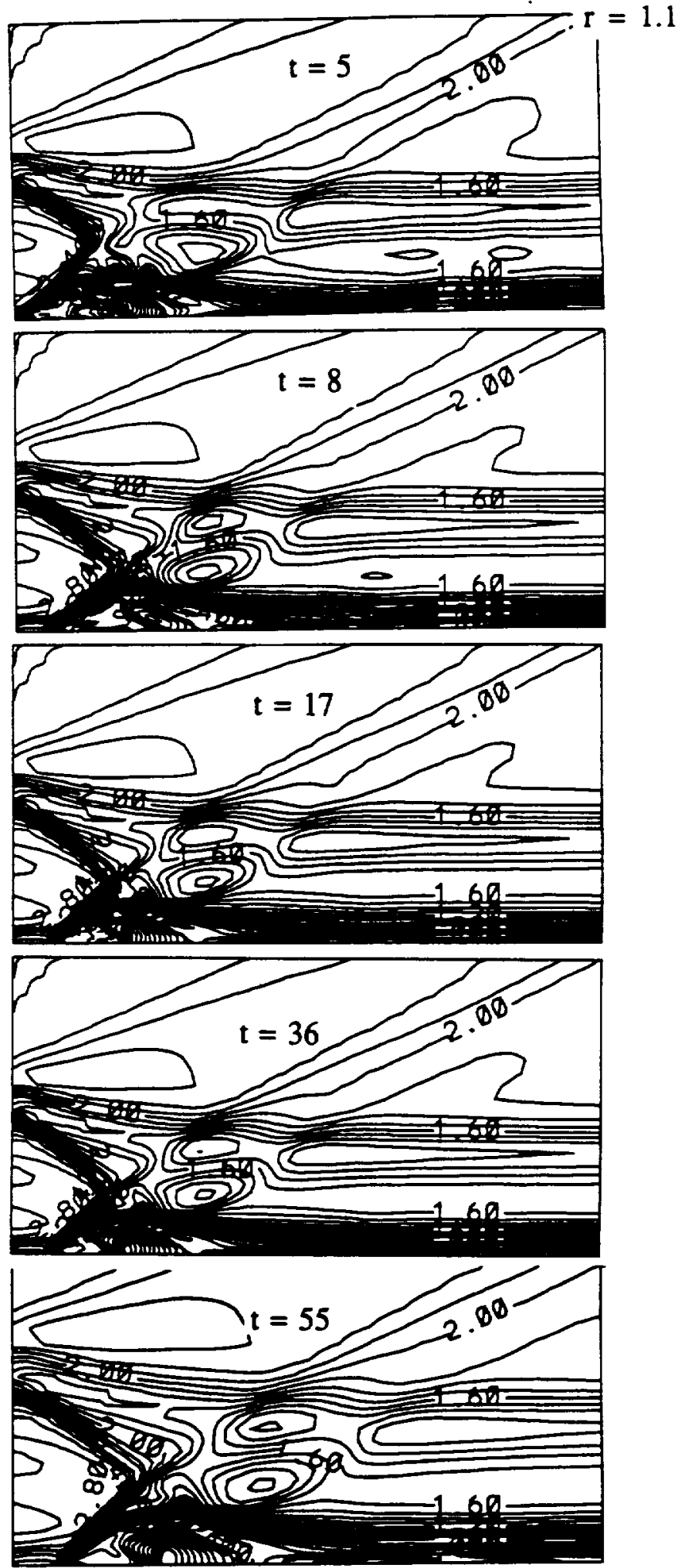

Figure 10. Streamlines and Mach contours for supersonic swirling jet from a nozzle with lowfrequency almost single bubble vortex breakdown, extrapolation from interior B.C. 
$\mathrm{r}=0.695$
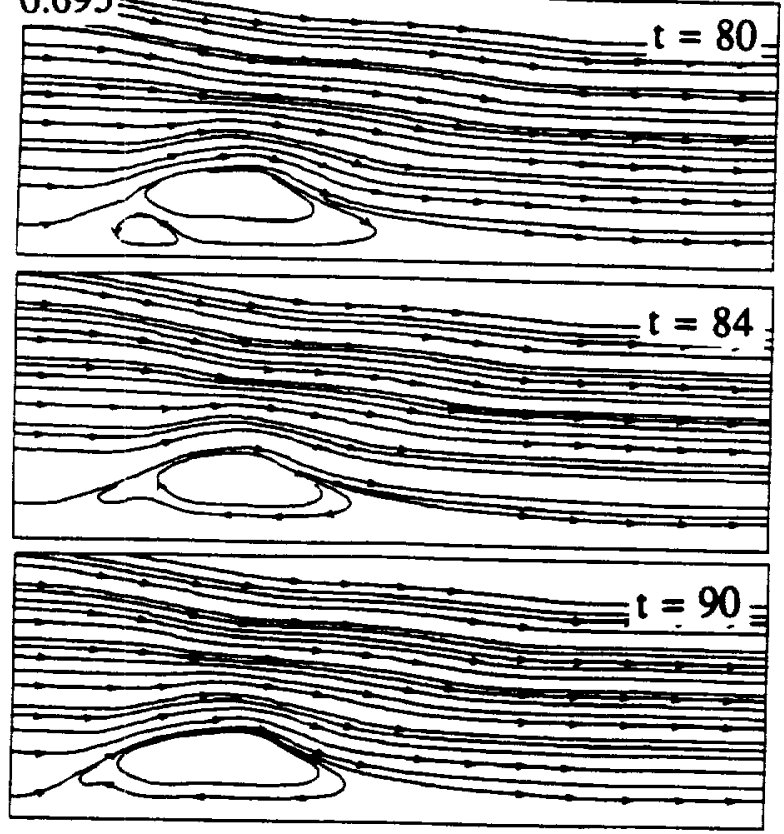
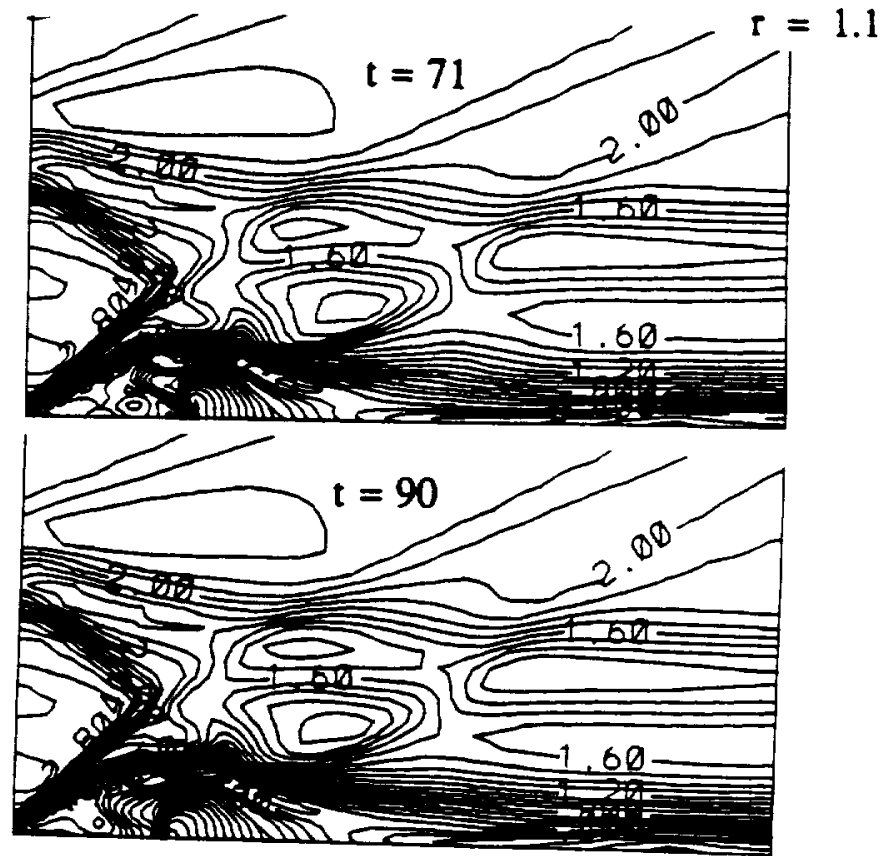

Figure 10. Continued

\section{$r=0.695$}
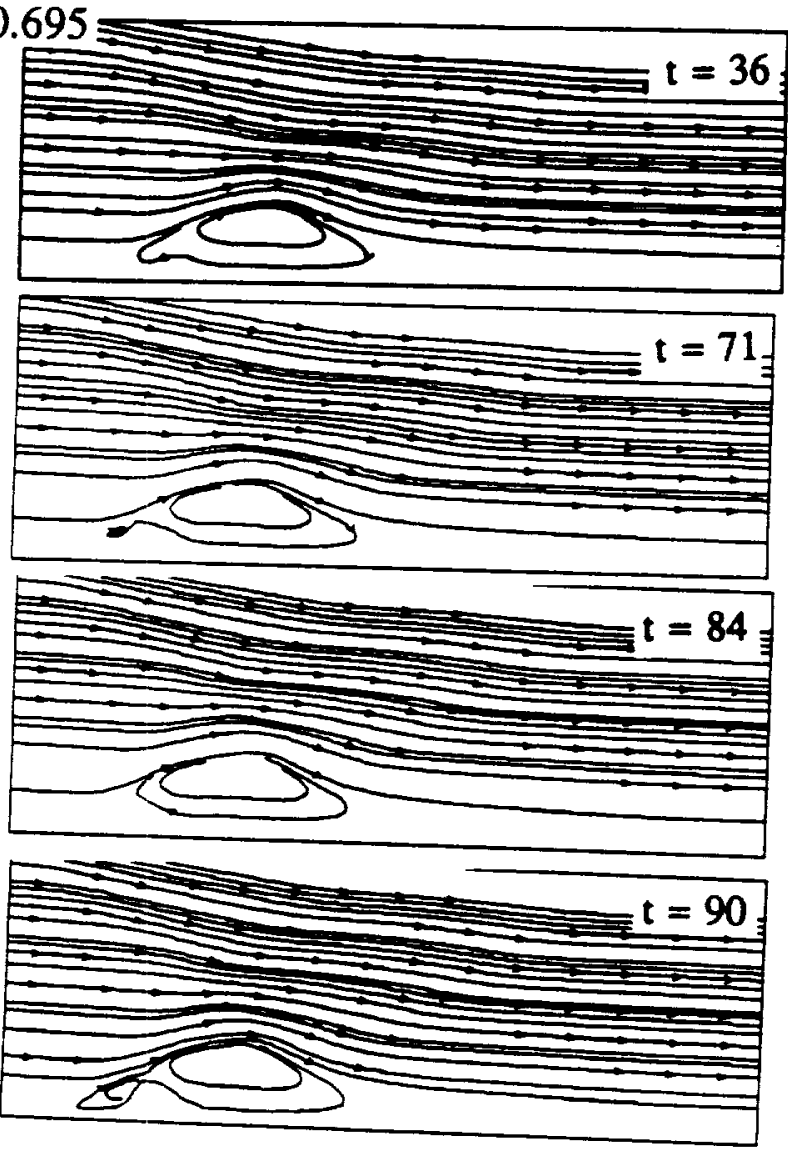
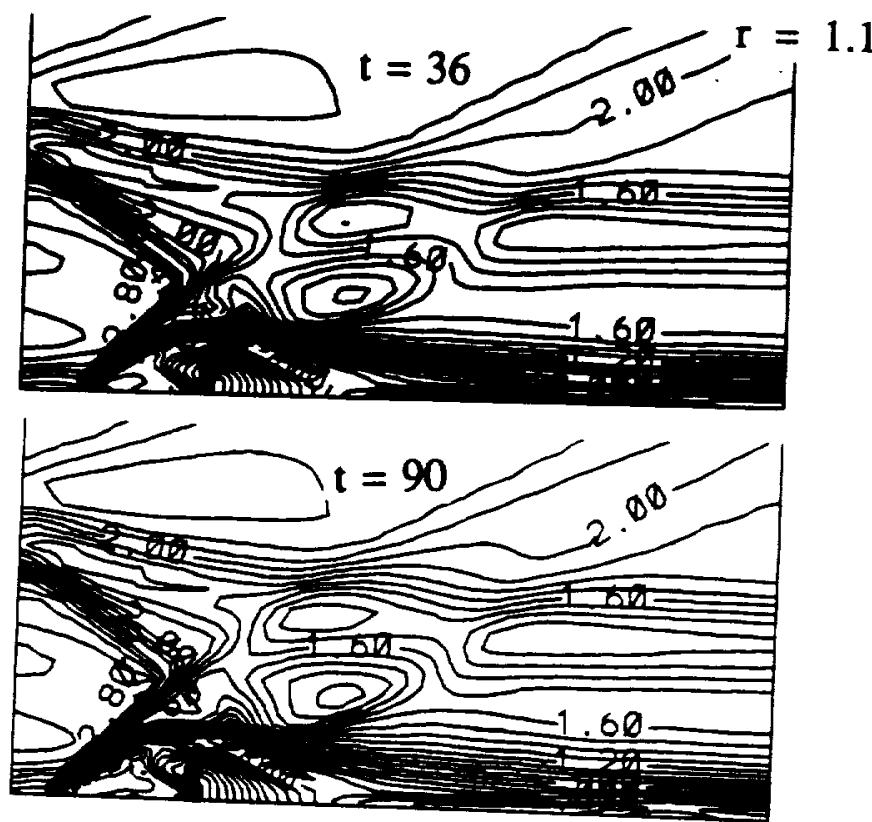

Figure 11. Streamlines and Mach contours for supersonic swirling jet from a nozzle with low frequency almost single bubble vortex breakdown, Riemann invariant B.C. 


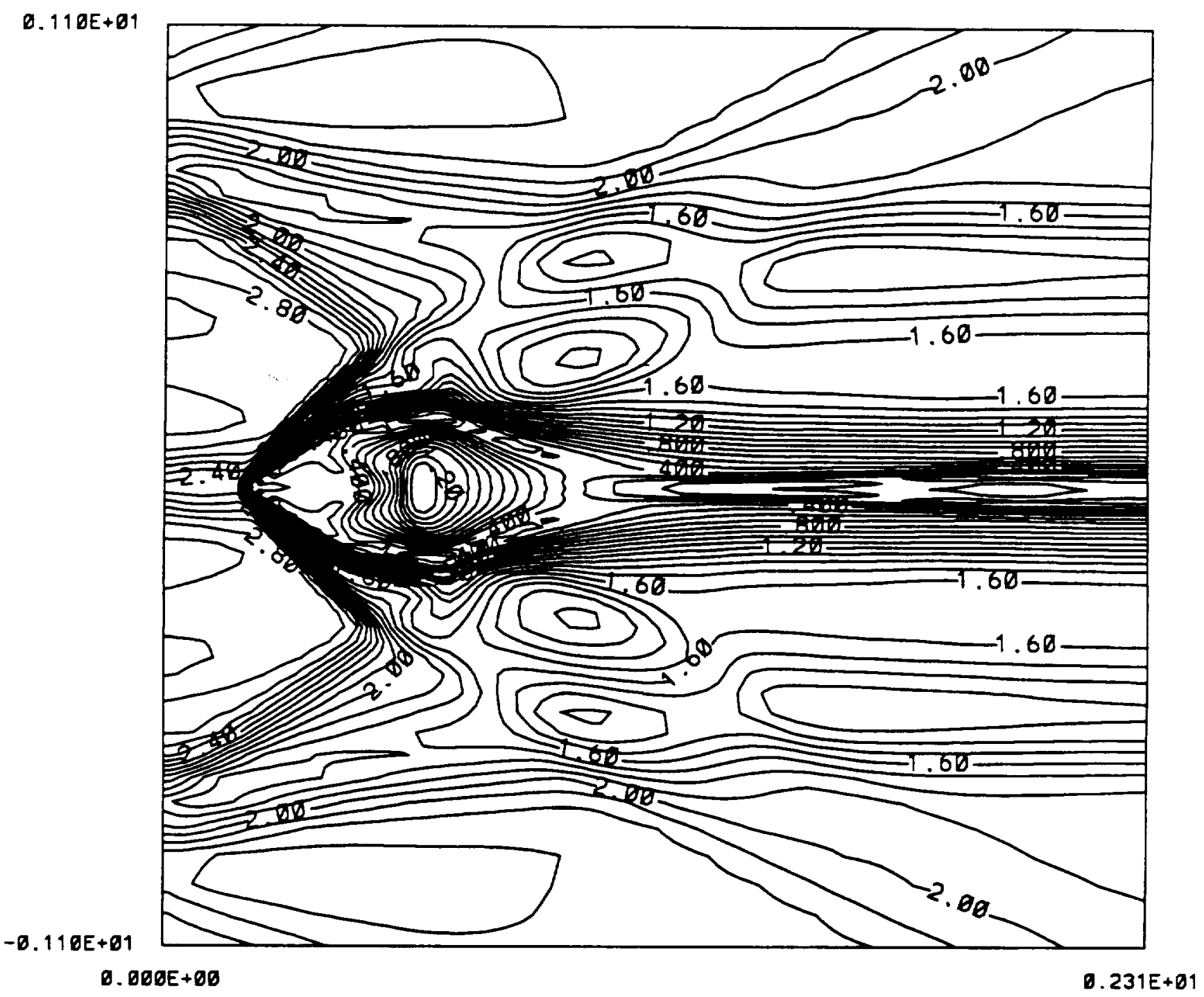

Figure 12. Blow-up of Mach contours at $t=55$ for supersonic swirling jet from a nozzle with low frequency almost single bubble vortex breakdown, Riemann invariant B.C. 
- 